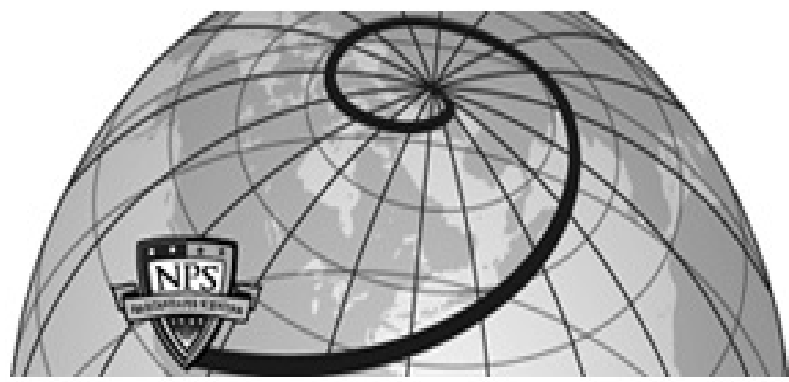

Calhoun: The NPS Institutional Archive DSpace Repository

1978-12

\title{
Impulse wave diffraction by rigid wedges and plates
}

\author{
Bremhorst, Joseph Henry
}

Monterey, California. Naval Postgraduate School

http://hdl.handle.net/10945/27092

This publication is a work of the U.S. Government as defined in Title 17, United States Code, Section 101. Copyright protection is not available for this work in the United States.

Downloaded from NPS Archive: Calhoun

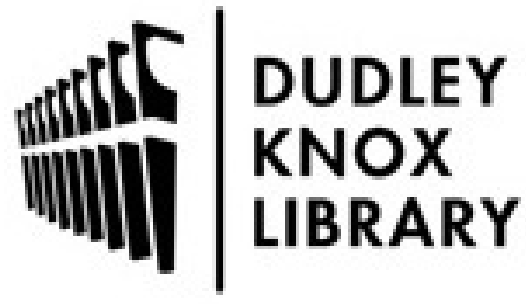

http://www.nps.edu/library
Calhoun is the Naval Postgraduate School's public access digital repository for research materials and institutional publications created by the NPS community. Calhoun is named for Professor of Mathematics Guy K. Calhoun, NPS's first appointed -- and published -- scholarly author.

Dudley Knox Library / Naval Postgraduate School 411 Dyer Road / 1 University Circle Monterey, California USA 93943 
$\therefore \quad \because n+2$ 
DUDiEY KinY LiU Th

MAYAL POST GRAOUATE SCHOOL

MONTEREY, CA 93940 





\section{NAVAL POSTGRADUATE SGHOOL Monterey, California}

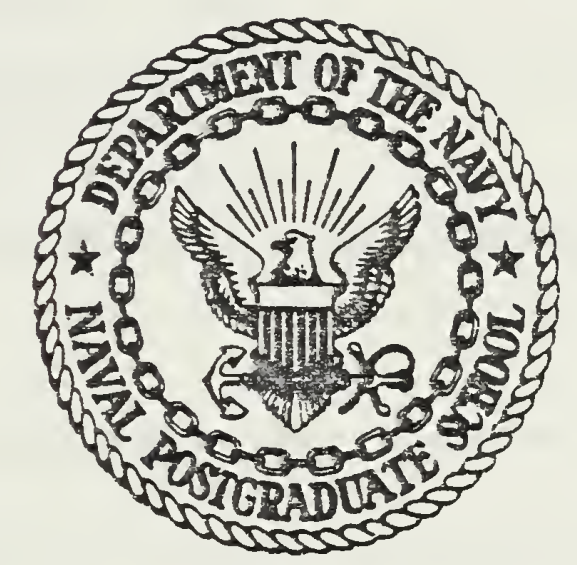

\section{THESIS}

IMPULSE WAVE DIFFRACTION

BY RIGID WEDGES AND PIATES

by

Joseph Henry Bremhorst

December 1978

Thesis Advisor:

H. Medwin

Approved for public release; distribution unlimited. 



\begin{tabular}{|c|c|}
\hline \multicolumn{2}{|c|}{ REPORT DOCUMENTATION PAGE } \\
\hline T. REPOAT NUMEER & 2. GOVT ACCESSION NO. \\
\hline \multicolumn{2}{|l|}{ 6. TITLE (and Sublillo) } \\
\hline \multicolumn{2}{|c|}{$\begin{array}{l}\text { Impulse Wave Diffraction by Rigid } \\
\text { Wedges and Plates }\end{array}$} \\
\hline \multicolumn{2}{|l|}{ 7. AUTHOA(D) } \\
\hline \multicolumn{2}{|l|}{ Joseph Henry Bremhorst } \\
\hline \multicolumn{2}{|l|}{ D. PERFORMING ORGAMIZATION NAME AND ADOAEBS } \\
\hline \multirow{2}{*}{\multicolumn{2}{|c|}{$\begin{array}{l}\text { Naval Postgraduate School } \\
\text { Monterey, California } 93940\end{array}$}} \\
\hline & \\
\hline \multicolumn{2}{|l|}{ 11. COMTROLLING OFEICE NAME AND ADORESS } \\
\hline \multicolumn{2}{|l|}{ Naval Postgraduate School } \\
\hline Monterey, California 93940 & \\
\hline
\end{tabular}

READ INSTRUCTIONS

3. TYPE OF AEPORT PEAIOD COVERED Master's Thesis;

December 1978

3. DEROAMIMG OAG. AEPOAT NUMEEA

8. COMTAACT OA GAANT NLMEEA(D)

10. PROGAAM ELEMENT, PROJECT, TASK AREA WORK UNIT NUMEERS

12. AEPOAT DATE

December 1978

13. NUMEER OF PAGES 113

19. SECURITY CLASS. (OO InIO paport)

Unclassified

T3. DECLASBIFICATION/DOWNGRADING
SCMEDUL

16. DISTRIBUTION STATEMENT (OL thle Ropore)

Approved for public release; distribution unlimited.

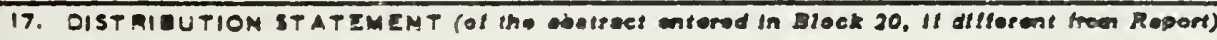

18. SUPQLEMENTARY NOTES

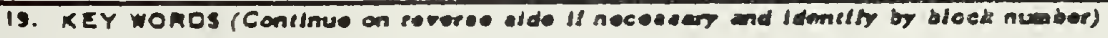

Impulse Wave Diffraction

Acoustic Signal Diffraction

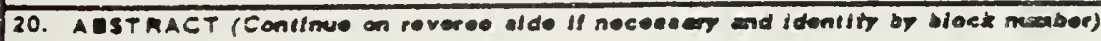

The problem of diffraction of acoustic signals by rigid barriers is studied empirically. Backward and forward diffraction from a 90 degree wedge and a thin plate are analyzed. Attempts to measure the diffracted energy in the illuminated region over the apex of the barrier, where direct and reflected signals coexist with diffracted, are discussed. Factors influencing the choice of the barriers' physical dimensions and composition are listed, as

DD, SORM 1473

(Page 1)
UNCLASSIFIED 

ABSTRACT (Cont'd)

are the problems surrounding the selection of an "ideal" sound source and receiver. Finally, the data are compared to the theory by Biot and Tolstoy (Journal of the Acoustical Society of America, v. 29, 1957) and found to be in good agreement with predictions. 



\section{Impulse Wave Diffraction}

by Rigid Wedges and Plates

$$
\text { by }
$$

Joseph Henry Bremhorst

Lieutenant Commander, United States Navy

B.S., St. Ambrose College, 1968

Submitted in partial fulfillment of the requirements for the degree of

MASTER OF SCIENCE IN ENGINEERING ACOUSTICS

from the

NAVAL POSTGRADUATE SCHOOL

December 1978 



\section{ABSTRACT}

The problem of diffraction of acoustic signals by rigid barriers is studied empirically. Backward and forward diffraction from a 90 degree wedge and a thin plate are analyzed. Attempts to measure the diffracted energy in the illuminated region over the apex of the barrier, where direct and reflected signals coexist with diffracted, are discussed. Factors influencing the choice of the barriers' physical dimensions and composition are listed, as are the problems surrounding the selection of an "ideal" sound source and receiver. Finally, the data are compared to the theory by Biot and Tolstoy (Journal of the Acoustical Society of America, v. 29, 1957) and found to be in good agreement with predictions. 

I. INTRODUCTION

II. RESEARCH FACILITIES -

A. ANECHOIC CHAMBER -

B. DATA ACQUISITION AND PROCESSING EQUIPMENT --- 10

C. MISCELLANEOUS EQUIPMENT -

III. THEORY - 18

IV. THE EXPERIMENT - 28

A. SELECTION OF ACOUSTIC SOURCE -

B. RECEIVER SELECTION - 38

C. THE DESIGN OF THE MODELS -

1. Considerations for the Weage --.---- 41

2. Considerations for the Plate - 47

D. SOURCE SIGNAL PARAMETERS - 49

E. RECEIVED SIGNAL ANALYSIS - 54

V. EXPERIMENTAL RESULTS - 60

A. FORWARD DIFFRACTION FROM WEDGE -..-.-.- 60

B. BACKWARD DIFERACTION FROM WEDGE

C. UPWARD DIFFRACTION FROM WEDGE --_-- 72

D. Z-AXIS DEPENDENCY -

E. FORWARD DIFFRACTION FROM PLATE - 88

F. BACKWARD DIFFRACTION FROM PLATE -.-.-- 90

G. CONCLUSIONS - 108

VI. RECOMMENDATIONS FOR FUTURE RESEARCH ---.----- 110

BIBLIOGRAPHY -

INITIAL DISTRIBUTION LIST - 



\section{ACKNOWLEDGMENT}

The author is indebted to his thesis advisor, Dr.

H. Medwin of the Naval Postgraduate School, for his continual support and guidance. Dr Medwin's expertise in scientific research provided the confidence and inspiration which lit many dark passages in the course of this endeavor.

Many thanks are also due to Mrs. Jeanie Savage for making possible the digital processing in this research. Her ability to grasp the physical concepts involved is surpassed only by her superb computer programing skills. The most capable assistance of technicians $k$. Smith, R. Moeller, and $T$. Maris is greatly appreciated. The financial support of the Office of Naval Research (Code 480) is acknowledged. 



\section{INTRODUCTION}

The problem of diffraction of acoustic energy by barriers has been studied extensively from the theoretical standpoint, with numerous papers concerning the subject published in the literature over the years. Very little has been done however to examine the problem experimentally. That is the purpose of the present work: To study firsthand the nature of sound diffracted by barriers and to validate the theories.

Significant contributions to the theoretical aspects of the problem have been made by Biot and Tolstoy, Tuzhilin, Oberhettinger, and Pierce [Refs. 1,2,3, and 4]. Of these, the Biot-Tolstoy theory has the most to offer. Biot \& Tolstoy in the early 1950 's, were the first to depart from more conventional methods of solving the diffraction problem by applying the concept of the normal modes of vibration of the system, and, by developing the method of nomal coordinates, they solved the problem of the diffraction of point source radiation by an infinite rigid wedge or corner [Ref. 1]. The method of normal coordinates and the solution to this problem by Biot and Tolstoy are outlined in the section on theory.

H. G. Jonasson undertook a theoretical as well as experimental development of diffraction by wedges, publishing his results in 1972 [Ref. 5]. His theoretical approach 

involves making numerous assumptions and applying them to existing solutions which use the method of images. The experimental data which Jonasson obtained provides the most recent basis for comparison to other experiments, albeit over a very limited frequency range and only one geometry.

The section on research facilities describes the anechoic chamber at the Naval Postgraduate School, the data acquisition and processing capabilities of the Ocean Physics Environmental Effects Analyzer (OPHELEA) system, and the miscellaneous equipment used in the experiment.

The section on experimental procedures describes the rationale for the selection of a sound source which most closely approximates a "point" source over a very wide range of Erequencies. Also, the considerations for selection of a receiver are listed, as are the constraints immediately placed on the experiment by choosing specific receivers. Additionally, this section provides the basis for the design of the model barriers, both wedge and plate, and the Iimitations imposed by certain designs. The intent here is to choose barriers which approximate infinite dimensions as seen from the wavelength of the acoustic signal, and which approximate a perfectly rigid plane surface. Finally, in this section, the signal sent to the source and the procedures for processing and analyzing the received signal are discussed. 
The experimental results are presented and compared to the Biot-Tolstoy theory for different cases. These cases include: forward diffraction from the 90 degree wedge; backward diffraction from the 90 degree wedge; forward diffraction from the thin plate; backward diffraction from the thin plate; and the response of the diffracted energy as the probe receiver moves along the apex of the wedge away from the plane of the source. Also in the section on experimental results is described the attempt to measure precisely the diffracted signal which theory predicts exists over the apex of the barrier, but which is masked by the greater energy arriving at the receiver from the direct path propagation and from the reflection off the front face of the barrier. These signals; direct, reflected, and diffracted, arrive at the receiver, positioned in the illuminated region over the barrier, at approximately the same time, making their superposition difficult to deconvolve. 



\section{RESEARCH FACILITIES}

\section{A. ANECHOIC CHAMBER}

The anechoic chamber provides isolation from external noise sources, absorption of internal sounds, and atmospheric stability. Noise transmission from outside is minimized by a "floating" room-within-a-room construction. The outer 12 inch concrete-walled room is separated from the inner room of concrete-block sides and floor by a 2 inch blanket of fiberglass and cork. Fiberglass wedges are attached to the walls, ceiling and floor of the inner room. These wedges are 40 inches deep, composed of P.F. 612 fiberglass, and have a total volume of approximately 5000 cubic feet.

Reflection of sounds from the side walls is minimized by the fiberglass wedges which are designed to trap and absorb sounds incident upon them. The 40 inch wedges absorb approximately $99 \%$ of the incident sound of frequency greater than 100 Hertz. The floor is a grid of 225 wire cables each stretched at a tension of 150 to 200 pounds per square inch. The size of the usable region within the chamber is $27 \times 14 \times 11$ feet high.

B. DATA ACQUISITION AND PROCESSING EQUIPMENT Data acquisition and processing were accomplished by utilizing a digital computer system composed of four primary 

components each of which is interfaced to provide high speed analog to digital conversion, digital processing, and data printout and recording. The design was developed by the Special Projects Section of the Naval Air Development Center in conjunction with Pinkerton Computer consultants, Inc., of Warminster, Pennsylvania. The components are pictured in figure 1 and described below.

1. Interdata Model 70 Computer

This minicomputer is a digital design that is FORTRAN and BASIC programmable with a 64 thousand byte memory. In addition to actual core memory, data that have been stored on diskettes, (floppy discs), or digital tape cassettes can be read into the computer for processing.

2. Phoenix Analog to Digital Converter, Model ADC 912 The Model ADC 912 is a very high speed, high accuracy analog voltage to digital converter capable of encoding \pm 10 volt input signals into twelve binary bits of data, with a resolution of one part in 4,095, at the maximum rate of 2 microseconds per conversion. It measures the input voltage against the internai precision reference voltage source with an accuracy of $=0.025 \%$ of full range. The fast settling time and the successive approximation encoding process will accommodate a typical commutating through-put rate of 476,190 channels per second. The sampling frequency is sent to the converter from an external oscillator providing a series of rectangular pulses. The 



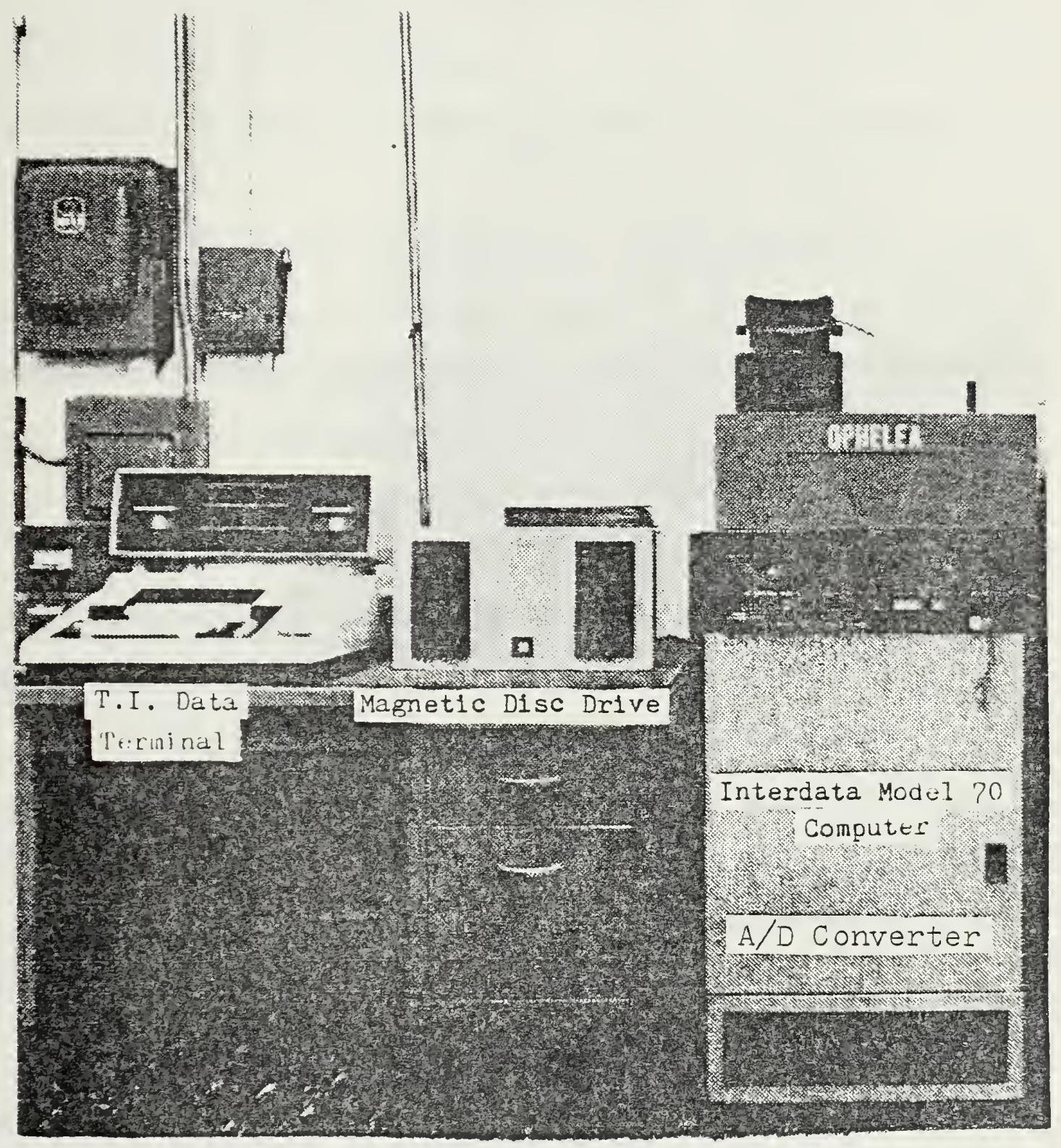

Figure 1. Digital Computer System 

leading edge of the rectangular pulse, a transition from the logical "O" level to the logical "l" level, initiates the encoding process. The minimum duration of the true state is 200 nanoseconds and the minimum duration of the false state is 200 nanoseconds. The true, logical "I", rectangular pulse, or command to convert, is +5 volts, maximum.

The converter performs two basic functions; 1) it measures the input voltage by comparing it against the internal reference voltage and 2) it converts the measurement into a digital number.

3. Orbis Diskette Drive, Model 76S

The Model $76 \mathrm{~S}$ diskette drive is a peripheral device interfaced with the Interdata 70 Minicomputer. Its functional characteristics are the ability to read and write on a standard diskette, or floppy disc. The dual drive unit utilizes industry standard diskettes which provide 3.2 milion bits of data with a data rate of 250,000 bits per second encoding for each drive. The unit is capable of a 6 millisecond access time track-to-track.

The storage element, or diskette, is a ferromagnetic coated flexible disk enclosed within a protective plastic jacket, 8 inches by 8 inches. The element has one recording surface, 77 tracks per surface, with a track spacing of 48 tracks per inch. 

4. Texas Instrument Electronic Data Terminal, Model 733 The Silent 700 electronic data terminal, Model 733, manufactured by Texas Instruments Incorporated, is a keyboard send-receive unit with selectable transmission speeds of 110, 150, and 300 baud $(10,15$ or 30 characters per second). The 733 is designed in several modular units: the keyboard, printer mechanism, and transmit/receive electronics.

\section{STANDARD EQUIPMENT LIST}

Much of the equipment used was standard off-the-shelf scientific measurement equipment. A list of the standard equipment used in the experiment and referred to in the text is given below:

Interface Technology Timing Simulator/Word Generator Model RS -648

Tektronix Four-trace Oscilloscope Model 545B

Wavetek Wave Generator Model 114

Hewlett-Packard Electronic Counter Model 5223L

Donner Frequency/Period Meter Model 8050

Hewlett-Packard Dual-trace Oscilloscope Model 140A

Fluke True RMS Voltmeter Model 8920A

Fluke Digital Multimeter Model $8000 \mathrm{~A}$

North Hills Wide-band Transformer

Lambda Regulated Power Supply

Krohn-Hite Frequency Filter Model 3550 (two)

Princeton Applied Research Amplifier Model 113 

Bruel and Kjaer Microphone Cartridge Model 4145

Bruel and Kjaer Microphone Cartiridge Model 4133 (two)

Bruel and Kjaer Microphone Preamplifier Model 2619

Bruel and Kjaer Microphone Power Supply Model 2804

Some of these pieces of equipment are shown in a typical rack mounting in figure 2. The equipment diagram for the experiment is shown in figure 3 . 



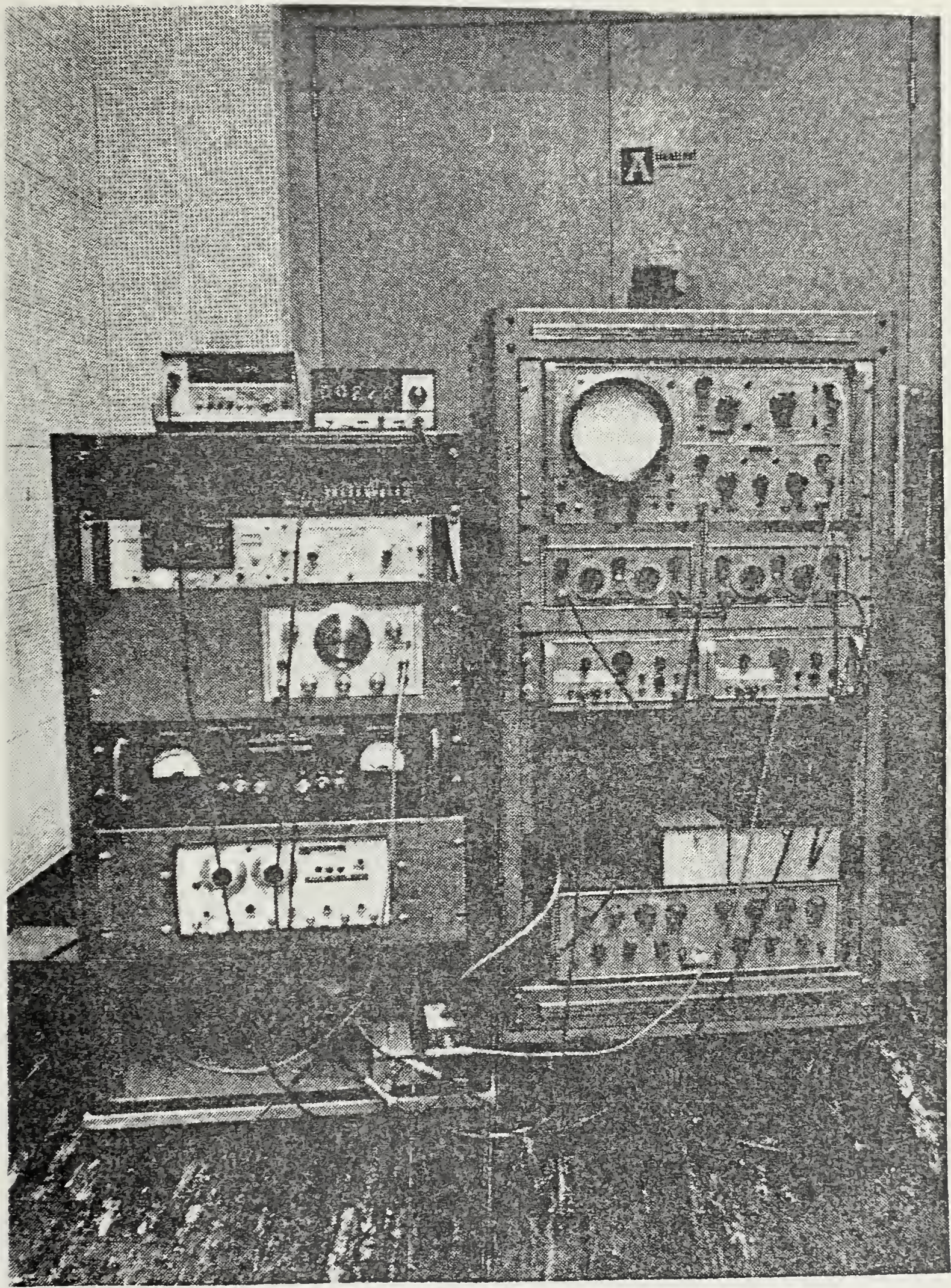

Figure 2. Typical Rack Mounting 



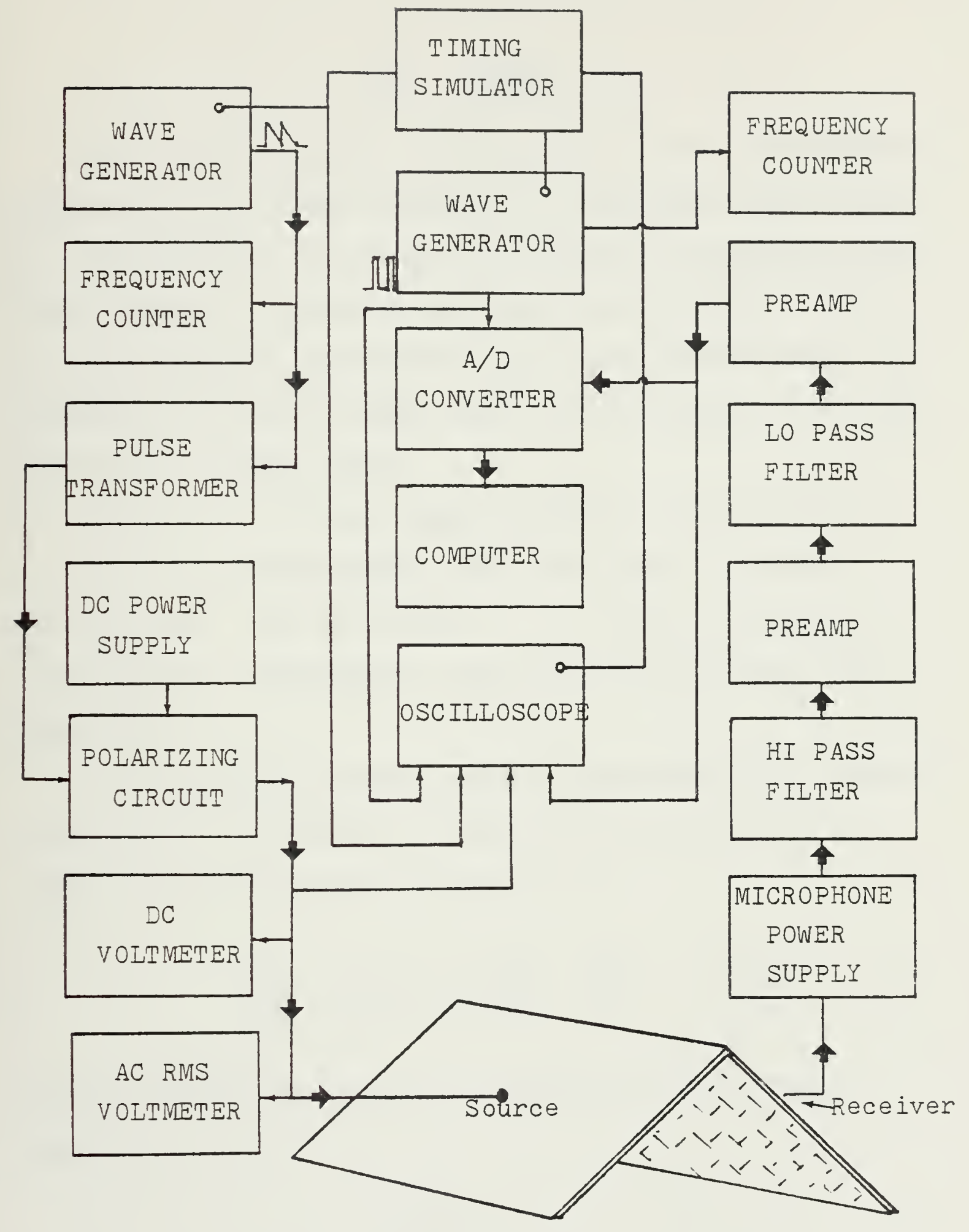

Figure 3. Equipment Diagram 



\section{THEORY}

Biot and Tolstoy apply the method of normal coordinates to the solution of the problem of a transient point source in a wedge-shaped region having perfectly reflecting walls. Their solution is briefly outlined here in order to provide the physical assumptions upon which the mathematical foundation is laid and thus show the link between the model experiment and the theory.

Consider an infinite wedge bounded by rigid planes $\theta=0, \theta=\theta_{w}$ intersecting along the z-axis, or apex of the wedge. Let the region $0<\theta<\theta_{w}$ be occupied by a homogeneous compressible fluid of sound velocity $c$ and density $\rho$.

To discover the normal modes of the system the acoustic wave equation is solved in cylindrical coordinates, in terms of the displacement potential $\phi$ :

$$
\frac{\partial^{2} \phi}{\partial r^{2}}+\frac{1}{r} \frac{\partial \phi}{\partial r}+\frac{1}{r^{2}} \frac{\partial^{2} \phi}{\partial \theta^{2}}+\frac{\partial^{2} \phi}{\partial z^{2}}=\frac{1}{c^{2}} \frac{\partial^{2} \phi}{\partial t^{2}}
$$

The displacement potential is related to the acoustic pressure, $p$, by

$$
p=-\rho \frac{\partial^{2} \phi}{\partial t^{2}}
$$



Solutions to equation (1) are of the form

$$
0=e^{ \pm i \nu \theta_{H}}(I, 2)(K r) e^{ \pm i \gamma z} e^{ \pm i \omega t}
$$

where $<, \gamma$, and $w$ are the separation constants and are related by

$$
\omega=\mathrm{kc}
$$

and

$$
k=\sqrt{k^{2}+\gamma^{2}} .
$$

$\gamma$ may be thought of as the $z$ component of the wave propagation vector $k$ and $<$ as the radial component.

The first term on the Ris of equation (3) can be written

$$
e^{ \pm i v \theta}=A \cos v \theta+B \sin v \theta
$$

Since the imaginary part of the Hankel function must become infinite at $r=0$, a situation not apolicable to this pinsically motivated problem, the second term on the RHS of equation (3) can be written simply as the Bessel function of the Eirst kind, that is, the real part of $\mathrm{H}_{v}(1,2)$. From normal coordinate methods the amplitudes, which are functions of time, are taken as generalized coorainates, 

the space-dependent part is the normal mode. If the particle displacements are given by $\eta_{i}$, then the transformation is

$$
\eta_{i}=q a_{i}
$$

where the index $i$ describes the tensor notation of space variables, and the $a_{i^{\prime}}$ (functions of $x, y, z$ ), form a fixed eigenvector in $\mathrm{n}$ space, and the $q$ are functions of time.

Now the last term in equation (3) can be replaced by $q$ as a function of time and the solution to equation (1) becomes

$$
\phi=q(A \cos v \theta+B \sin v \theta) J_{v}(K r) e^{ \pm i \gamma z}
$$

where q obeys the equation

$$
\ddot{q}+\omega^{2} q=Q(t),
$$

$Q$ being the generalized exciting force.

Applying the boundary conditions wherein the sides of the wedge are perfectly rigid, requiring the displacements normal to the sides to vanish, that is

$$
\frac{\partial \phi}{\partial \theta}=0 \text { at } \quad \theta=0, \theta=\theta_{W}
$$

leaves the solution in the form 



$$
\phi_{n}=q_{n} \cos \nu_{n} \theta J_{v}(k r) e^{ \pm i \gamma z}
$$

$\mathrm{n}$ being the mode number and

$$
\nu_{n}=\frac{n \pi}{\theta_{w}}
$$

Biot and Tolstoy now assume an explosive point source located at $r=r_{0} ; \theta=\theta_{0} ; z=0$ allowing only solutions symmetric in $z$. The relevant modes are thus

$$
\begin{aligned}
& \phi_{n}=q_{n} \psi_{n} \\
& \psi_{n}=J_{\nu_{n}}(\kappa r) \cos \gamma_{n} \theta \cos \gamma z
\end{aligned}
$$

Applying an orthonormality condition for a continuous, linear, conservative system, and an expression for an explosive point source corresponding to the injection of a unit volume, they arrive at an expression for the $q_{n}$

$$
q_{n}=\frac{\cos \omega t}{\omega^{2}} \frac{2 c^{2}}{\pi \theta_{w}} J_{\nu_{n}}\left(k r_{0}\right) \cos \nu_{n} \theta_{0} k d k d \gamma
$$

the terms $r_{0}$ and $\theta_{0}$ describe the position of the source. Substituting into equation (II) and summing,

$$
\begin{array}{r}
\rho=\frac{2 c^{2}}{\pi \theta_{w}} \sum_{n=0} \cos \nu_{n} \theta \cos \nu_{n} \theta_{0} \int_{0}^{\infty} \int_{0}^{\infty} J_{\nu_{n}}(k r) J_{\nu_{n}}(k r) \\
\\
\times \cos \gamma z \frac{\cos \omega t}{\omega^{2}} \kappa d k d \gamma
\end{array}
$$



and

$$
\begin{gathered}
\frac{\partial \phi}{\partial t}=-\frac{2 c^{2}}{\pi \theta_{w}} \sum_{n=0} \cos \nu_{n} \theta \cos \nu_{n} \theta \int_{0}^{\infty} \int_{0}^{\infty} J_{\nu_{n}}(k r) J_{\nu_{n}}\left(k r_{0}\right) \\
\times \cos \gamma z \frac{\sin \omega t}{\omega} \kappa d k d \gamma
\end{gathered}
$$

To do the integration with respect to $\gamma$ a transform familiar to propagation problems is helpful [Ref. 6]:

$$
\int_{0}^{\infty} \cos \gamma z \frac{\sin c t\left(\gamma^{2}+\kappa^{2}\right)^{\frac{1}{2}}}{\left(\gamma^{2}+\kappa^{2}\right)^{1 / 2}} d \gamma=0_{0 ; c t<z}^{\frac{\pi}{2} J_{0}\left[k\left(c^{2} t^{2}-z^{2}\right)^{\frac{1}{2}}\right] ; c t>z}
$$

using the aforementioned relationships (equations 4 and

$$
\omega=c \sqrt{k^{2}+\gamma^{2}}
$$

Then

$$
\frac{\partial \phi}{\partial t}=-\frac{c}{\theta_{W}} \sum_{n} \cos v_{n} \theta \cos \nu_{n} \theta_{0} I_{n} ; c t \geq z
$$

where 



$$
I_{n}=\int_{0}^{\infty} J_{v_{n}}(k r) J_{v_{n}}\left(k r_{0}\right) J_{0}\left[k\left(c^{2} t^{2}-z^{2}\right)^{\frac{1}{2}}\right] k d k
$$

Now, let

$$
\begin{aligned}
& t_{0}=\frac{1}{c}\left[(r-r)^{2}+z^{2}\right]^{1 / 2} \\
& t^{\prime}=\frac{1}{c}\left[\left(r+r_{0}\right)^{2}+z^{2}\right]^{1 / 2}
\end{aligned}
$$

where $t_{0}$ is the time of arrival of the first direct pulse from source to receiver and $t^{\prime}$ is the earliest arrival having traveled from source to apex to receiver. The integral of equation (18) will have values for $t<t_{0}$; $t_{0}<t<t^{\prime}$ and $t>t^{\prime}$.

For $t<t_{0}: I_{n}=0$ as would be expected. For $t_{0}<t<t^{\prime}$ the solution describes the acoustic field for the direct and reflected energy, corresponding to the solution achieved from the method of images.

The case for $t \geq t^{\prime}$ is the solution of interest to this study. This is a closed form solution completely describing the diffracted energy. For $t>t^{\prime}$ :

$$
I_{n}=-\frac{1}{\pi r r_{0} \sinh n} \sin \frac{n \pi}{\theta_{w}} \pi e^{-n \pi n / \theta_{w}}
$$

where

$$
n=\operatorname{Arg} \cosh \frac{c^{2} t^{2}-\left(r^{2}+r_{0}{ }^{2}+z^{2}\right)}{2 r r_{0}}
$$



Using the solution to equation (20) for $t>t^{\prime}$ in equation (17),

$$
\frac{\partial \phi}{\partial t}=\frac{c}{\pi \theta_{w}} \frac{1}{r r_{0} \sinh n} \sum_{n=0}^{\infty} \cos \nu_{n} \theta_{0} \cos \nu_{n} \theta \sin \nu_{n} \pi e^{-\nu_{n} n}
$$

recalling

$$
v_{n}=\frac{n \pi}{\theta_{w}}
$$

and writing

$$
\begin{aligned}
\cos \nu_{n} \theta 0 \cos \nu_{n} \theta \sin \nu_{n} \pi= & \frac{1}{8 i}\left(e^{i \nu_{n}{ }^{\theta} o_{+}}+e^{-i \nu_{n}{ }^{\theta}}\right)\left(e^{i \nu_{n}{ }^{\theta}}+e^{-i \nu_{n} \theta}\right) \\
& \times\left(e^{i \nu_{n} \pi^{-i \nu_{n} \pi}}\right)
\end{aligned}
$$

Then summing equation (22) and collecting conjugate pairs results in

$$
\frac{\partial \phi}{\partial t}=\frac{c}{4 \pi \theta_{w}} \frac{1}{r r_{0} \sinh n} e^{-\pi n / \theta_{w}}\left[\frac{\sin \left(\pi / \theta_{w}\right)\left(\pi \pm \theta_{0}\right)}{1-2 e^{-\pi n / \theta_{w_{0}}} \cos \left(\pi / \theta_{w}\right)\left(\pi \pm \theta_{ \pm} \theta_{0}\right)+e^{-2 \pi n / \theta_{w}}}\right]
$$

where the \pm signs indicate that the form in brackets is actually a sum of four terms corresponding to all possible combinations of these signs. 

By applying equation (2), where the acoustic pressure

is

$$
\mathrm{p}=-\rho \frac{\partial^{2} \phi}{\partial t^{2}}
$$

the pressure due to the diffracted wave alone is then

$$
p=-\frac{\rho c}{4 \pi \theta_{w}} \frac{1}{r r_{0} \sinh n} e^{-\pi n / \theta_{w}}\left[\frac{\sin \left(\pi / \theta_{w}\right)\left(\pi \pm \theta \pm \theta_{0}\right)}{1-2 e^{-\pi n / \theta_{w}} \cos \left(\frac{\pi}{\theta_{w}}\right)\left(\pi \pm \theta_{0} \theta_{0}\right)+e^{-2 \pi n / \theta_{w}}}\right]
$$

where the total diffracted wave is the sum of all combinations of the \pm signs, and where

$\theta_{w}=$ fluid filled region outside of wedge

$\theta_{0}=$ angle from side of wedge to the source

$\theta=$ angle from side of wedge to receiver

$r_{0}=$ distance from source to apex of wedge

$r=$ distance from apex to receiver

$z$ = distance along apex of wedge.

See figure 4.

Since the frequency domain solution to the impulse wave diffraction problem is more familiar to the applied acoustician, equation (25) has been transformed by Medwin [Ref. 7] 



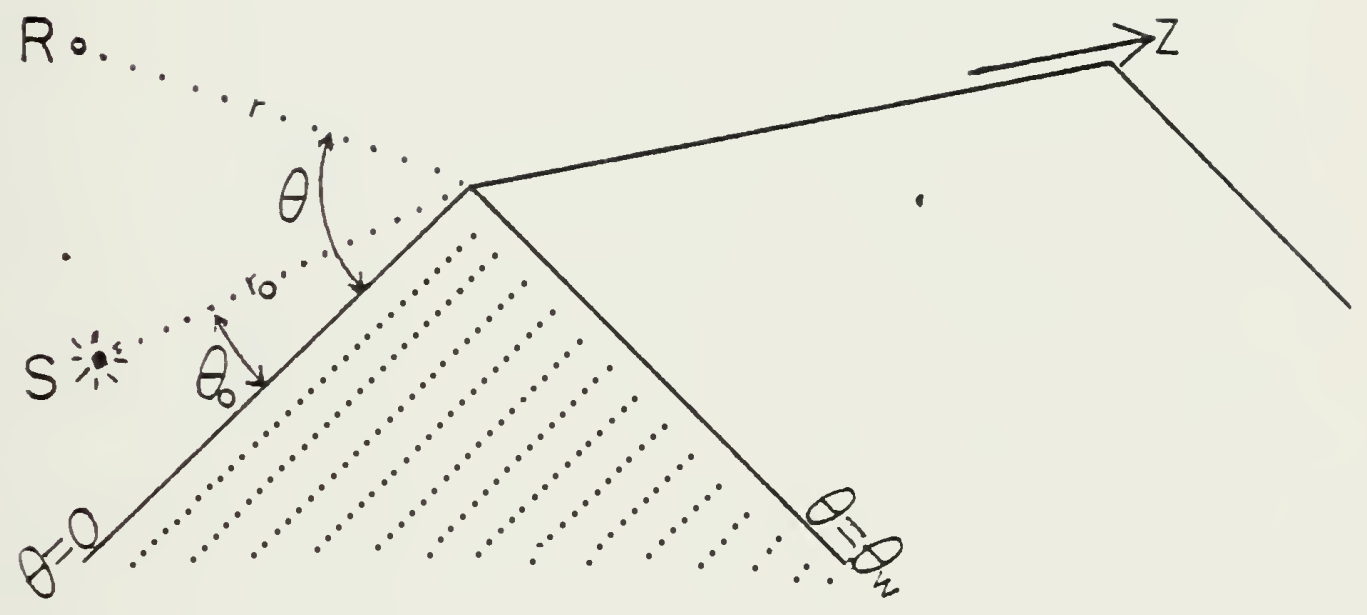

Figure 4. Source $S$ at $r=r, \theta=\theta, z=0$; Receiver $R$ at radlal distance $r$ and angle $\theta$ in a fluid filled region extending from $\theta=0$ to $\theta=\theta_{W}$. 

to the frequency domain. The results of his work provide the theoretical values with which the experimental data is compared in section $V$. 



\section{THE EXPERIMENT}

\section{A. SELECTION OF THE SOUND SOURCE}

The sound source used in this experiment would ideally be a point source generating spherical acoustic waves. To set up harmonic spherical waves in the surrounding medium, which is considered to be homogeneous and isotropic, a pulsating sphere is preferred, but impractical.

Acoustic sources do indeed act like pulsating spheres, or simple sources, at least to a first approximation, if their dimensions are small compared with the wavelength of the radiated signal. That is to say that the source may be considered a simple source when $\mathrm{ka}<1$, where the wave number $k=2 \pi / \lambda$, and $a$ is the radius of the source, and if $r>a$, where $r$ is the radial distance from the source.

For small distances from a point source of sound the particle velocities corresponding to even very low acoustic pressures become impossibly large with the result that a small source of sound is inherently incapable of generating spherical waves of large intensity. Similarly, it is impossible to construct a sound source of moderately small size that is capable of radiating large amounts of power at low frequencies.

Microphones today are manufactured in surprisingly small sizes. For example, Bruel and Kjaer, of Denmark, have developed a line of calibrated, condenser microphones in 

1, $\frac{1}{2}, \frac{1}{4}$, and $\frac{1}{8}$ inch diameter sizes, which have an active face (consisting of a thin nickel diaphragm stretched above the stainless-steel backplate) with diameters approximately $75 \%$ of the above outside dimensions. These condenser microphones, being reciprocal transducers, can be used advantageously as sound sources. They have the same welldefined properties when used as transmitters as when they are used as receivers, although the sound pressure levels are relatively low.

The B\&K microphones are readily available, and, because of their small size, appear to be ideally suited for use as simple sources. However, it must be noted that the active face is flat, vice spherical, and thus behaves more like a piston radiator, prejudicing the simple source requirement.

To examine this problem, allow the source strength to be equal to unity so that the acoustic pressure of a simple source radiating spherically can be written as

$$
p=\frac{j \rho_{0} c k}{4 \pi r} e^{j(\omega t-k r)}
$$

Now, if the spherical radiation is taken to be into an infinite half-space only, the pressure is simply

$$
p=\frac{j \rho_{0} c k}{2 \pi r} e^{j(\omega t-k r)}
$$



The equation for the acoustic pressure radiated by the piston source into an infinite half-space is

$$
p=\frac{j \rho_{0} c k}{2 \pi r} e^{j(\omega t-k r)}\left[\frac{2 J_{1}(k a \sin \theta)}{k a \sin \theta}\right]
$$

It is seen clearly, that the only difference between pressure radiating from a spherical source, given by equation (27), and that from a piston source, given by equation (28), is a directivity term

$$
D=\frac{2 J_{l}(k a \sin \theta)}{k a(\sin \theta)}
$$

Normalized plots of this directivity are presented in figures 5, 6, and 7 , for the $\frac{1}{2} " B \& K$ microphone at frequencies of $2.5,20$, and $40 \mathrm{kHz}$, respectively. Plotted on the same figures are experimentally acquired values for this microphone.

Since the pressure radiated from a piston source is a function of polar angle, as well as distance from the source, the wave is not spherically symmetrical. It still has, however, the characteristic property of a diverging spherical wave, namely, the pressure is inversely proportional to the radial distance from the center of the source. The phase of the pressure is also the same on any spherical wave front at all points included within the major lobe. It should now be clear that, in using a small, flat, condenser microphone.as a sound source, the resulting 



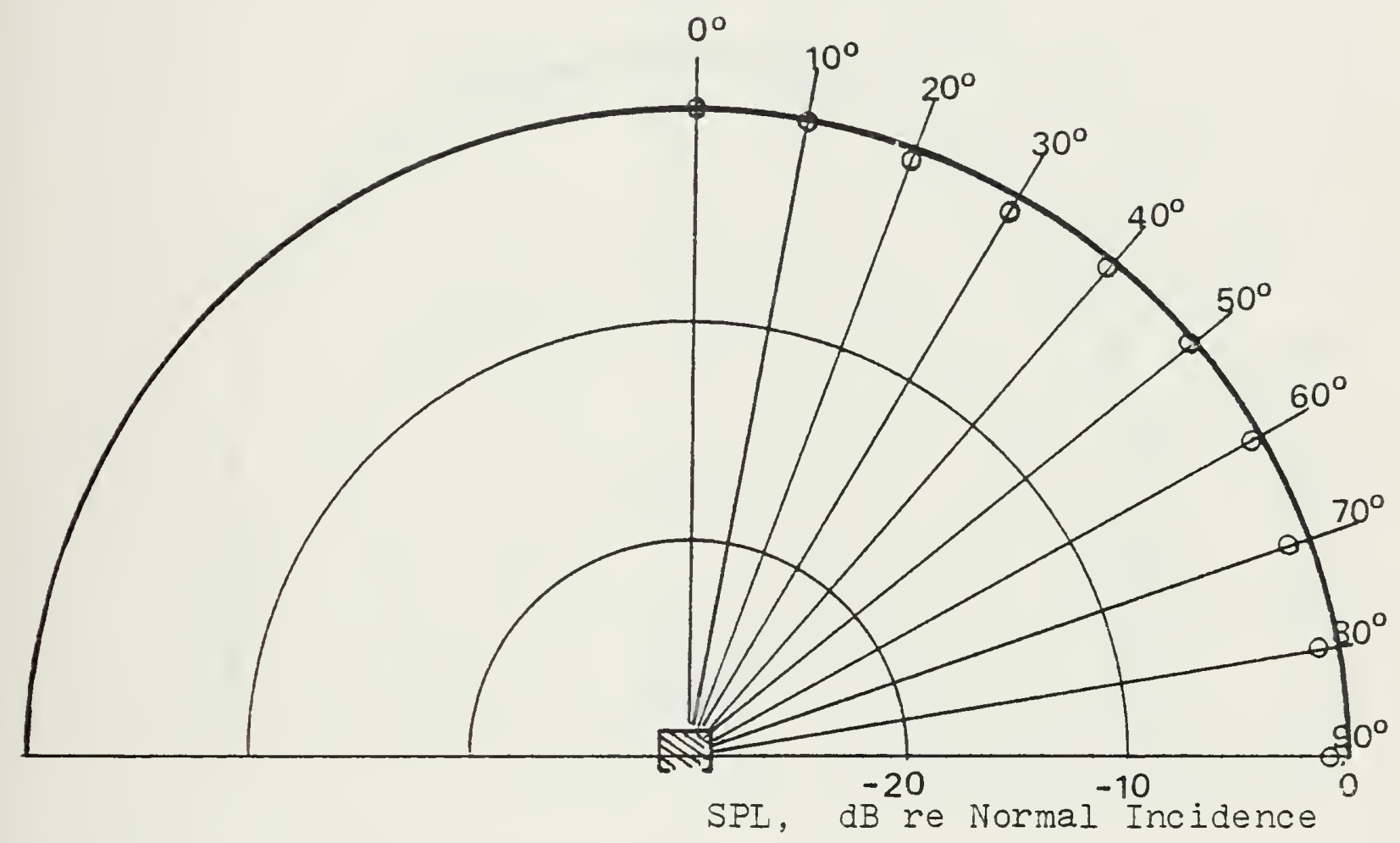

Figure 5. Polar Plot of $\frac{1}{2}$ " B\&K Microphone used as Transmitter at $2.5 \mathrm{kHz}$.

Theoretical: - Actual: 0 



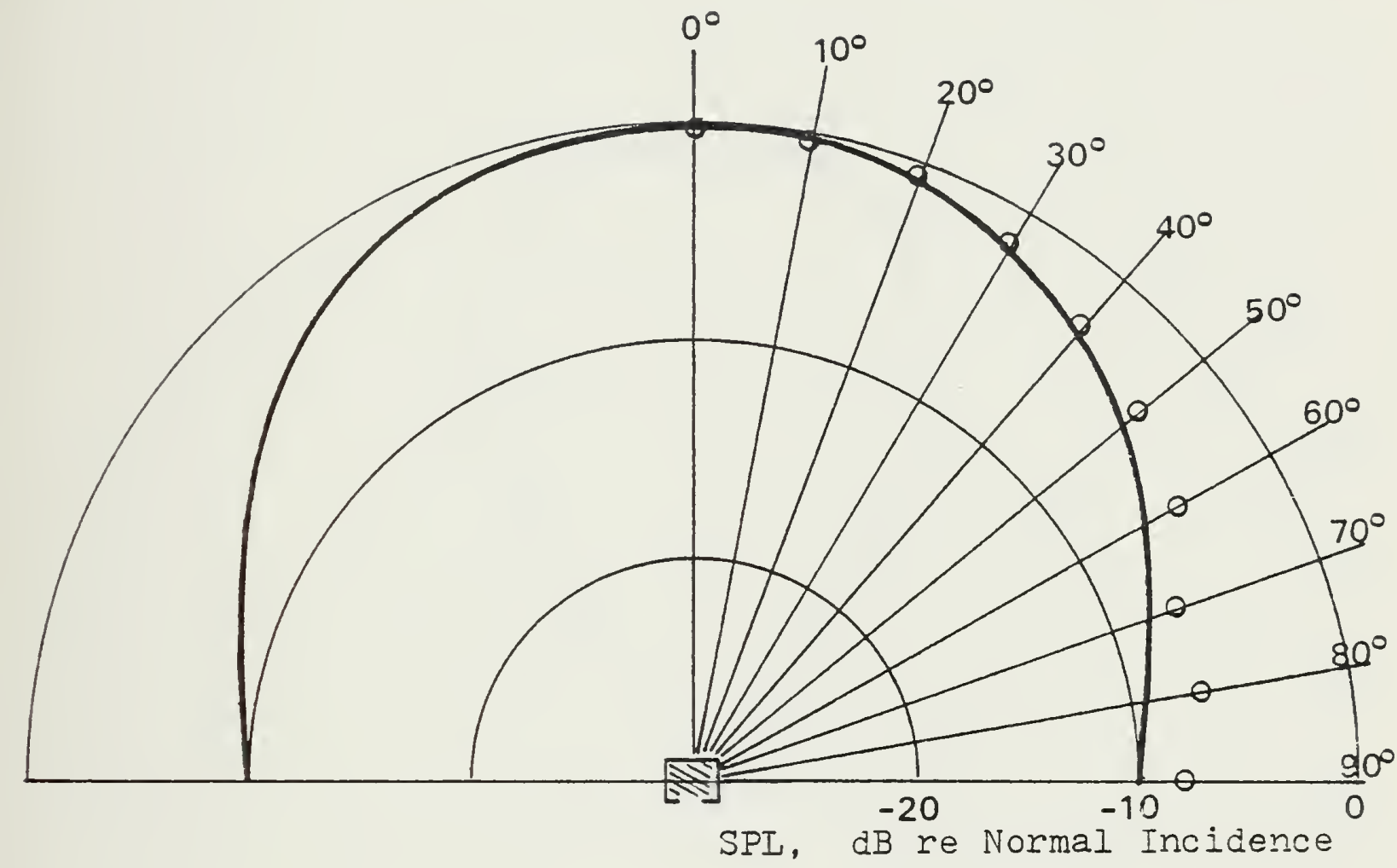

Figure 6. Polar Plot of $\frac{1}{2} "$ B\&K Microphone used as Transmitter at $20 \mathrm{kHz}$.

Theoretical:

Actual: 0 



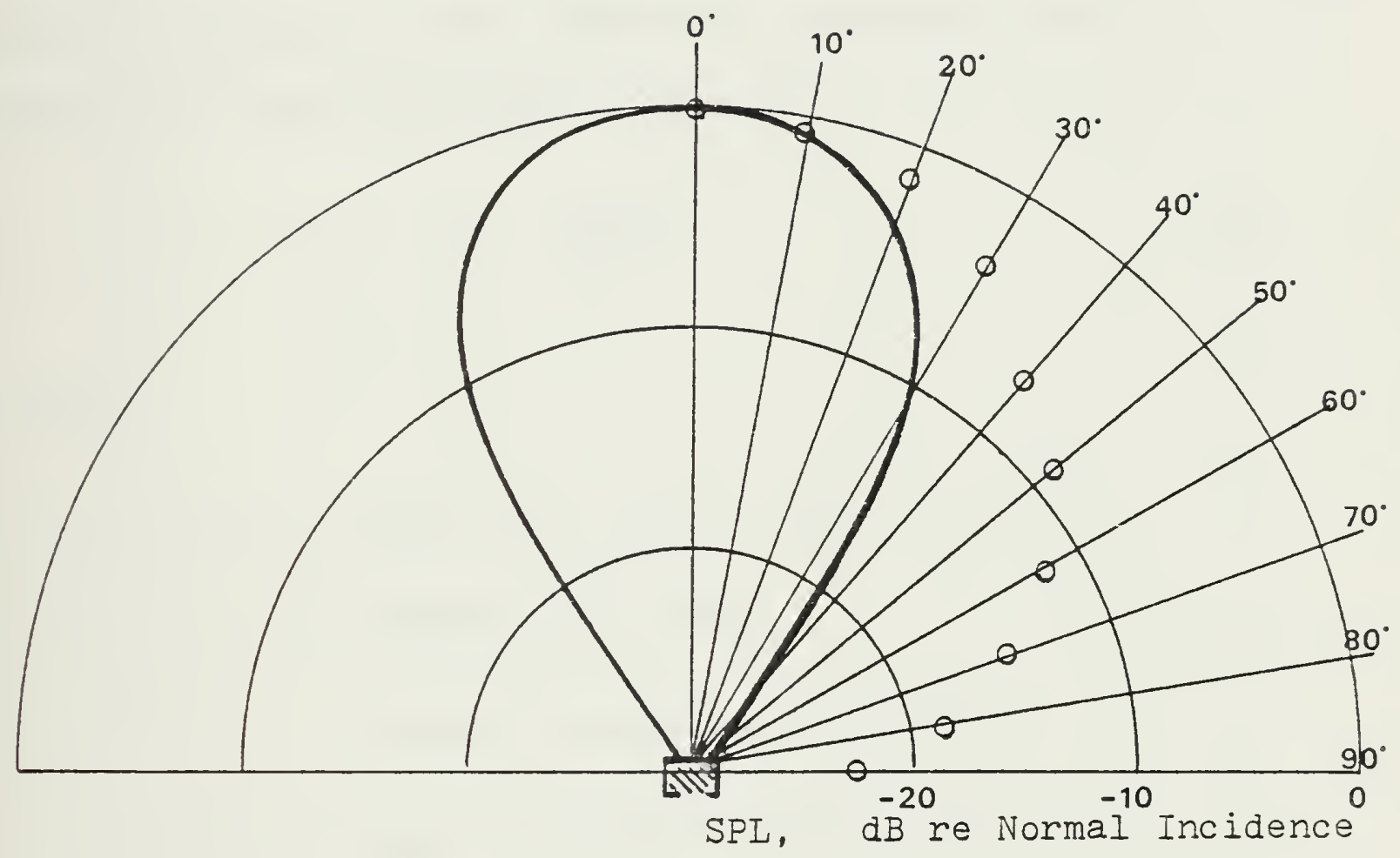

Figure 7. Polar Plot of $\frac{1}{2} "$ B\&K Microphone used as Transmitter at $40 \mathrm{kHz}$.

Theoretical: - Actual: o 

pressure waves are indeed spherical. However, an attempt must be made to aim the source in order that the target, in this case the apex of the barrier, lies on or near the axis of the source.

Using the reciprocity theorem, the modulus of the sound pressure generated by the condenser microphone at a certain distance, under free-field conditions, can be determined from the formula [Ref. 8]

$$
p=\frac{\rho_{0} f M i}{2 d}
$$

where

$$
\begin{aligned}
& \rho_{0}=\text { density of } \operatorname{air}\left(1.2 \mathrm{~kg} / \mathrm{m}^{3} \text { at } 20^{\circ} \mathrm{C}\right) \\
& f \quad=\text { frequency in Hertz } \\
& i \quad=\text { current input into microphone } \\
& M \quad=\text { free field sensitivity }(\mathrm{V} / \mathrm{Pa}) \\
& \mathrm{d}=\text { distance from source. }
\end{aligned}
$$

Since a condenser microphone cartridge has a high impedance it is difficult to control the current applied to the microphone on account of stray capacitances. It is therefore practical to apply a fixed voltage across the terminals of the microphone and determine the sound pressure by substituting into equation (30) 

where

$$
\begin{aligned}
& \mathrm{e}=\text { voltage }(\mathrm{V}) \\
& \mathrm{z}=\text { impedance } \\
& \omega=\text { angular frequency } \\
& \mathrm{C}=\text { electrical capacitance of microphone }
\end{aligned}
$$

The pressure is then given by

$$
p=\frac{\pi \rho_{0} f^{2} \mathrm{MeC}}{d}
$$

Plots of pressure for different microphones are given in figure 8. By the above equation and the corresponding plots, the slopes of the curves can be seen to be $40 \mathrm{~dB} /$ decade. Most microphone cartridges have constant sensitivity, M, approximately up the diaphragm resonance frequency. Above this frequency the diaphragm is mass controlled causing $40 \mathrm{~dB} /$ decade decay. Thus one would expect the response of the transmitting microphone to be flat in this frequency region. This is not generally the case, however, since there are some resonances present caused by the standing waves inside the microphone housing and higher order modes of the diaphragm.

An important consideration when using a condenser microphone as a sound source, or receiver, is the necessity 



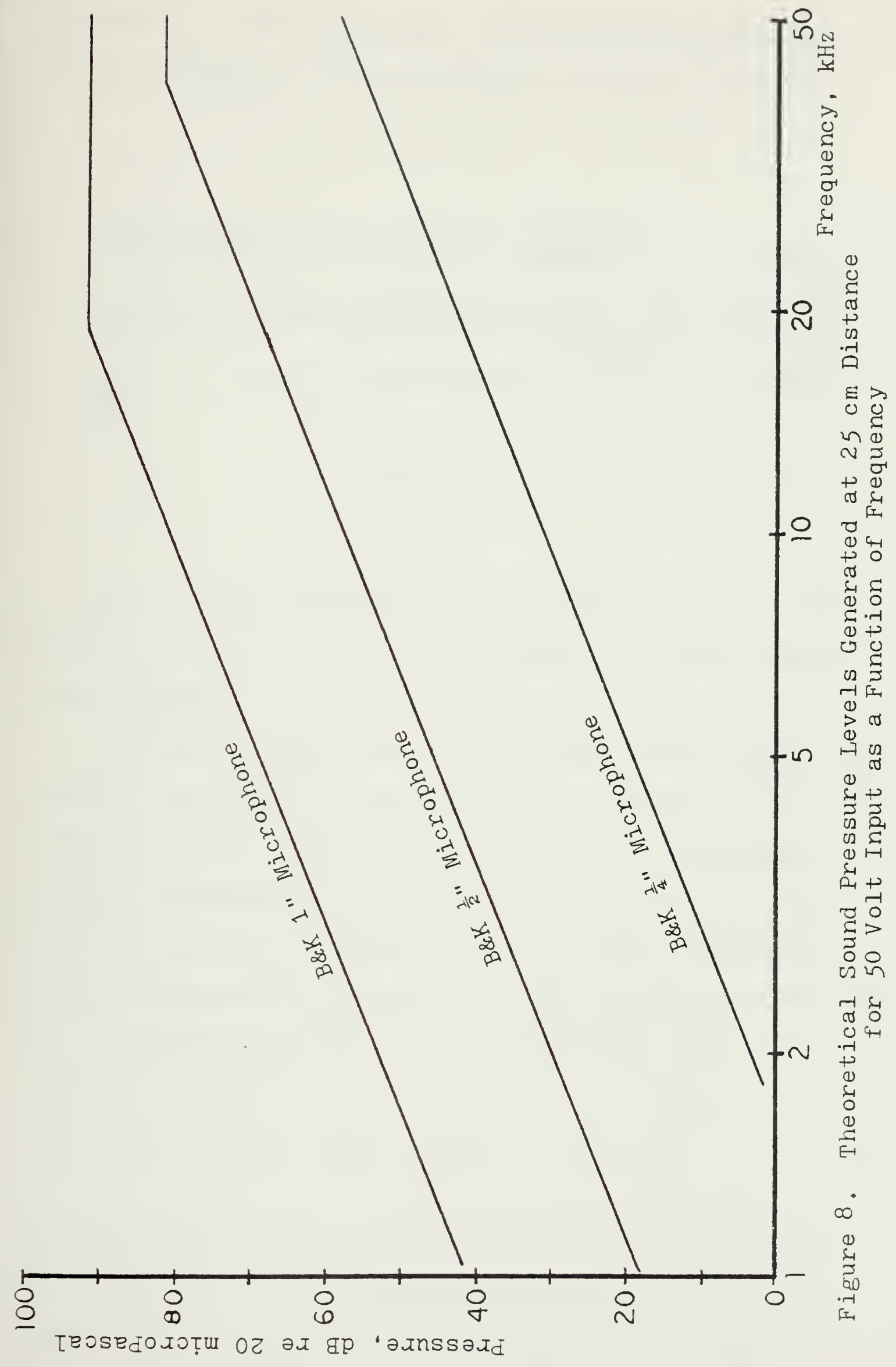


of polarizing the diaphragm. The electrical terminals of the microphone can be connected as shown in figure 9.

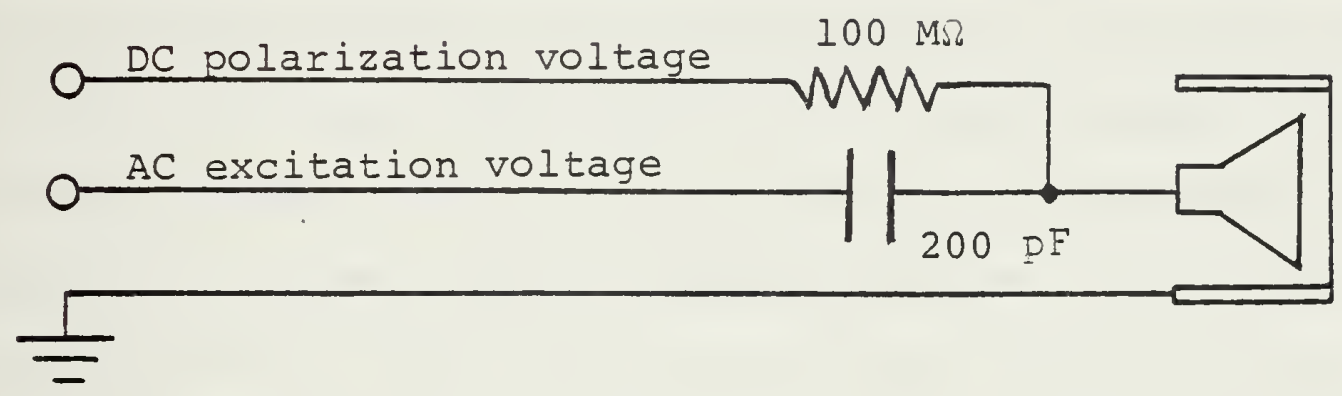

Figure 9

This circuit protects the microphone diaphragm from damage in the event of a short circuit or arcing within the microphone.

In order to avoid distortion in the output signal and minimize the affect on the microphone sensitivity and frequency response, the diaphragm must not be displaced too far from its equilibrium position. To accomplish this, the AC excitation voltage, e, must be small compared to the DC polarization voltage, $\mathrm{E}_{\mathrm{O}}$. The percentage distortion, $\mathrm{D}_{\mathrm{S}}$, of the output signal can be calculated from

$$
D_{S}=\frac{e}{4 E_{0}} \times 100 \%
$$

Reference 8 provides values of maximum limiting voltage, $D C+$ peak AC. For the one-inch microphone this value is 250V. For the one-half-inch microphone it is $300 \mathrm{~V}$. 

As can be seen from the foregoing information, there are many trade-offs in selecting an "ideal" source. In order to achieve the most acoustic pressure output from the source it was decided that both the one-inch B\&K 4145 and one-half-inch B\&K 4133 microphones could be used effectively. As can be seen from figure 8, and verified experimentally, smaller size microphones did not have sufficient output to achieve a usable signal-to-noise ratio. Yet, the dimensions of the active face of the larger one-inch and one-half-inch microphones are still small compared to a wavelength, an exception being the one-inch microphone at the very highest frequencies. Both microphones were used as sources during the course of the experiment.

The excitation voltage was kept to $50 \mathrm{~V}$ (peak) for both sources. However, when using the one-inch microphone, the DC polarization voltage was $200 \mathrm{~V}$, and when using the one-half-inch microphone, the DC polarization voltage was $250 \mathrm{~V}$. This gave signal distortions, according to equation (33), of $6.25 \%$ and $5 \%$ respectively.

\section{B. SELECTION OF THE RECEIVER}

In choosing the receiver to be used in measuring the acoustic field about the barrier three considerations become important; 1) microphone sensitivity, 2) size, and 3) frequency response. Once again, many trade-offs must be made to achieve a receiver suited to this experiment. 

In order to obtain the best signal-to-noise ratio, particularly in probing pressure fields created by the relatively weak outputs of the microphone sources described in the previous section, a microphone with a very high sensitivity is desired. However, the smaller the microphone the less sensitive it is.

The size of the microphone is extremely important when attempting to probe an acoustic field. Diffraction from the microphone itself can interfere extensively with the accuracy of the measurements. Here, the rule of thumb is; the smaller the microphone the less interference from diffraction caused by the microphone itself.

Additionally, frequency response of the probe microphone is important to this experiment since analysis at a wide range of frequencies is important. A desirable microphone would have a flat frequency response over the region of interest. Generally speaking, the smaller microphones have wider frequency ranges.

One more consideration becomes important to the experiment when matching condenser microphones to their associated preamplifiers. Here, the problem of self-noise generated by the preamplifier can become the limiting factor to the signalto-noise ratio. The preamplifiers designed for use with the smaller microphones are inherently more noisy than those used with the larger microphones.

After weighing the above factors and conducting many small experiments attempting to lower the receiver noise of 

the microphone-preamplifier combinations it was decided the best trade-off was a Bruel and Kjaer one-half-inch, No. 4133, microphone cartridge, B\&K No. 2619 preamplifier, and $B \& K$ No. 2804 battery operated power supply.

It can be seen then, that many of the considerations used in selecting the probe microphone are similar to those used in selecting the signal source. The final choice of one-half-inch receiver, its associated preamp and power supply, provided the best sensitivity with the necessary smallness, where the wavelengths of the signal are much larger than the dimensions of the microphone, and finally, a flat frequency response broad enough to cover the frequencies of interest.

In illustrating the sensitivity of the experiment to problems of this type, an older B\&K, No. 2615, preamplifier, compatible with the one-half-inch microphone, was used early in the data collecting part of the experiment. This particular preamplifier uses a small vacuum tube amplifier (located in the housing immediately behind the microphone cartridge), which generates a great deal of heat. It was found that this heat, emanating into the medium directly in front of the receiver, was the cause of considerable variability, of the order of $10 \mathrm{~dB}$, when attempting to repeat earlier results. The newer B\&K, No. 2619, preamplifier, using a FET amplifier in its front-end, was obtained and used in the experiment, virtually eliminating any fluctuations and allowing excellent repeatability of the measurements. 

This also illustrates reasons for the preference of the B\&K, No. 2804, battery operated power supply. In addition to being inherently less noisy than other similar power supplies, it does not provide power to the No. 2619 preamp heater element, and thus eliminated the requirement for disconnecting heater circuitry contained in other power supplies.

C. DESIGN OF THE MODELS

1. Considerations for the Wedge

To determine the composition of the wedge for the model experiment, a rigid, perfectly reflecting surface is desired. The goal here is to achieve a wedge constructed of a material which is inexpensive, accessible, easy to machine, and has a reflection coefficient as nearly equal to unity as possible.

Since a source of finite dimensions is used in this experiment, it must be assumed that the actual wavefront at the air-wedge interface is spherical. However, in studying the reflection coefficients it will suffice to use the plane wave approximation for non-dissipative fluids. The plane wave acoustic pressure reflection coefficient for fluids is known to be [Ref.9]

$$
R_{12}=\frac{\rho_{2} c_{2} \cos \theta_{1}-\rho_{1} c_{1} \cos \theta_{2}}{\rho_{2} c_{2} \cos \theta_{1}+\rho_{1} c_{1} \cos \theta_{2}},
$$



where $\theta_{1}$ is the angle of the incident wave measured from the normal to the surface, and $\theta_{2}$ is the angle of the transmitted wave in the second medium, the wedge, also measured from the normal to the surface. Taking the case of normal incidence, $\theta_{1}=\theta_{2}=0^{\circ}$, where the cosines of the angles are equal to one, gives

$$
R_{12}=\frac{\rho_{2} c_{2}-\rho_{1} c_{1}}{\rho_{2} c_{2}+\rho_{1} c_{1}} \text {. }
$$

A material available in large sheets, accessible, inexpensive, and easy to machine, is aluminum. The density of aluminum is $2700 \mathrm{~kg} / \mathrm{m}^{3}$ and the sound speed is $6300 \mathrm{~m} / \mathrm{s}$. The values for air at $20^{\circ} \mathrm{C}$, are: density = $1.21 \mathrm{~kg} / \mathrm{m}^{3}$ and sound speed $=343 \mathrm{~m} / \mathrm{s}$. Inserting these values into the above equation provides a reflection coefficient for aluminum of

$$
R_{12}=0.99995
$$

a value very near unity.

Another approach to choosing material for the model barrier is the mass action law [Ref. 10], wherein the amount of sound energy striking the barrier at normal incidence and transmitted through the material is determined by the mass of the material. A desirable material should have very little energy transmitted through it, a parameter 

which could be expressed in terms of transmission loss, in decibels. Here is needed a term referred to as the sound intensity transmission coefficient, $\alpha_{t} \cdot$ This term is the ratio of the transmitted flow of sound intensity to the incident flow of sound intensity, that is

$$
\alpha_{t}=\frac{I_{t}}{I_{i}}=\frac{4 \rho_{2} c_{2} \rho_{1} c_{1}}{\left(\rho_{2} c_{2}+\rho_{1} c_{1}\right)^{2}}
$$

Whenever $\rho_{2} C_{2}$ and $\rho_{1} C_{1}$ have widely separate magnitudes, the sound intensity transmission coefficient is small. Now the transmission loss can be written as

$$
\mathrm{TL}=10 \log \frac{I_{i}}{I_{t}}=-10 \log \alpha_{t}
$$

Inserting values for aluminum and air into the equations above, a transmission loss for aluminum is obtained as

$$
\mathrm{TL}=40 \mathrm{~dB}
$$

Consider sound energy traveling through three media, in this case from air into aluminum and then into air. Now the thickness of the aluminum becomes important. Here, the first and third media are the same, $\rho_{1} c_{1}=\rho_{3} c_{3}$, and $\rho_{2} c_{2} \gg \rho_{1} c_{1}$, thus the expression for the sound intensity transmission coefficient can be written as 



$$
\alpha_{t} \doteq \frac{4 \rho_{1}{ }^{2} c_{1}{ }^{2}}{\rho_{2} c_{2}{ }^{2} \sin ^{2} k_{2} 1}
$$

where $k_{2}$ is the wave number of the second medium (aluminum), and 1 is the thickness. This equation can be simplified even more by assuming that $k_{2} I<1$, ignoring very high frequencies and very large thicknesses, and by replacing the density and thickness terms by $\sigma$, where $\sigma$ is the area density of the second medium, in $\mathrm{kg} / \mathrm{m}^{2}$. Then

$$
\alpha_{t} \doteq\left(\frac{\rho_{1} c_{1}}{\pi \sigma f}\right)^{2}
$$

Now the transmission loss can be described by

$$
T L=20 \log \frac{\pi}{P_{1} C_{1}}+20 \log \sigma f
$$

For an aluminum sheet $6.35 \mathrm{~mm}$, ( $1 / 4$ inch),

thick,

$$
\sigma=17 \mathrm{~kg} / \mathrm{m}^{2}
$$

At the lowest frequency of interest, $2 \mathrm{kHz}$, the transmission loss is then

$$
\mathrm{TL}=48.2 \mathrm{~dB}
$$

and becomes larger with higher frequency. This transmission 

loss is enhanced even more in light of the fact that the $90^{\circ}$ wedge consists of two thickness of aluminum through which the sound energy must travel.

Obviously, the choice of aluminum as the material from which to construct the wedge is a good one. It is indeed accessible, easy to machine, has an extremely high reflection coefficient, and high transmission loss when compared to the air medium.

Due to the availability of specifically sized plates of aluminum, $\frac{1}{4}$ " thick, it was decided to construct the wedge from one plate sheared into two halves lengthwise, machined and joined at the apex to form a sharp $90^{\circ}$ angle. The result was a wedge 1.52 meters long ( $5 \mathrm{ft}$ ) and 0.6 meters $(2 \mathrm{ft})$ on the side. These dimensions somewhat restricted the .low frequency analysis, yet resulted in a convenient size for movement into the anechoic chamber. Two circular plates were attached to either end of the wedge (see figure 10), between which was strung fine wire to support the source. The extremely taut wire allowed for a firm support for the source, which could then be moved easily about the apex at constant radius by rotating the end-plates. Holes were drilled in the end-plates every five centimeters along the radius to facilitate moving the source forward and backward with respect to the apex, and thus take measurements at various distances from the apex.

The fine wires supporting the receiver were designed so as to avoid diffraction in the vicinity of the receiver, 



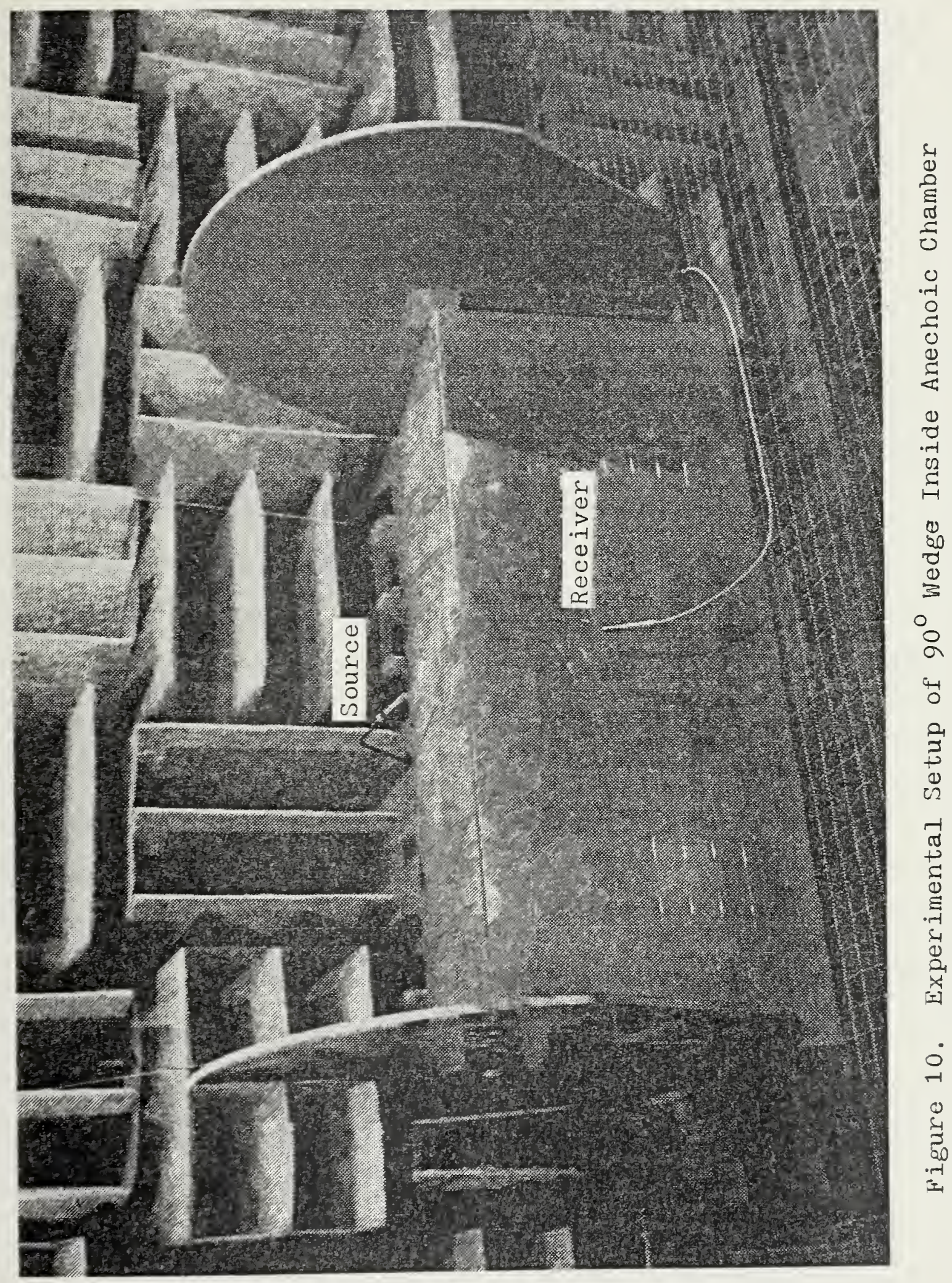



wherein the wire diameter was much, much less than the wavelength of the highest frequencies, thereby allowing no scattering of the acoustic field. The source was fixed rigidly to the wedge by means of a thin aluminum rod, approximately one-half centimeter in diameter positioned well behind the active face of the source so as not to interfere with the acoustic field propagating from it. The wedge was sufficiently long to allow a one cycle pulse at the lowest frequency of $2 \mathrm{kHz}$ to travel from the source to the apex to the receiver without interference from reflections off the end-plates. Additionally, the sides of the wedge were sufficiently deep, $(60 \mathrm{~cm})$, so that no energy was diffracted from the bottom of the wedge soon enough to interfere with the diffracted pulse from the apex. With these wedge dimensions and the pulsed signal technique, the wedge could be considered infinite with respect to the wavelengths of the frequencies of interest. Figure 10 shows a typical experimental setup inside the anechoic chamber.

\section{Considerations for the Plate}

The process of choosing the material and dimensions of the wedge outlined in the previous section, applies directly to the same parameters for the barrier. A few additional considerations enter in here, however. Unlike the wedge, the barrier consists of only one thickness of material, and transmission through the barrier is a more 

important factor. Hence, a barrier composed of a higher density material is desirable.

Another additional consideration is the thickness of the plate. Naturally, the thicker the plate, the higher the density per unit area, and the greater the transmission loss through it. However, the apex of the barrier must appear as an infinitely thin plate, not two 90 degree wedges, as the higher frequencies impinge upon it. Thus, a plate much thinner than $1 / 4$ of the wavelength is desirable. Finally, the rigidity of the material is important so as to avoid constructing additional supporting structure and thereby increasing interference from extraneous diffraction. A sufficiently rigid plate will allow tightening the wires supporting the receiver at a distance away from the apex, without bowing the plate along its length.

To fulfill more stringent requirements listed above for the barrier, a sheet of steel approximately $\frac{1}{2}$ centimeters ( $3 / 16$ of an inch) thick, 1.5 meters wide, and 2.5 meters long was appropriated. Here the area density for this $4.8 \mathrm{~mm}$ thickness of steel is

$$
\sigma=36.7 \mathrm{~kg} / \mathrm{m}^{2}
$$

and by equation (40) the transmission loss is then

$$
T L=55 \mathrm{~dB},
$$



almost $7 \mathrm{db}$ greater than the thicker aluminum, at $2 \mathrm{kHz}$ frequency. This thickness restricts the validity of the thin plate assumption to frequencies much less than $18 \mathrm{kHz}$. Above this, the wavelengths are sufficiently small to see the plate as two 90 degree wedges. It does, however, provide enough rigidity to support the endplates and wires.

\section{SOURCE SIGNAL PARAMETERS}

The waveform sent to the source to act as the acoustic signal had to be composed of many frequencies if the data was to be transformed to the frequency domain for streamlined analysis of the frequency behavior.

This objective was met easily by choosing the sawtooth waveform. The perfect sawtooth has an infinite slope on the positive going portion of the cycle providing the impulse, and, when transformed to the frequency domain by fast Fourier transform methods, provides frequencies at every harmonic of the fundamental sawtooth frequency. The amplitudes of these harmonic components decay at the rate of $6 \mathrm{~dB}$ per octave.

In generating the sawtooth wave electronically, however, the so-called "perfect" waveform is not achieved. The Wavetek Model 114 Frequency Generator was capable of a rise-time of 5 microseconds, the shortest rise-time of any available frequency generator capable of producing the sawtooth waveform. This rise-time is a good approximation of the infinitely small rise-time of an impulse when 

compared to a typical overall period of the wave, 500 microseconds. Figure 11 shows a digitized train of sawtooth pulses from the wavetek.

In order to boost the voltage output of the Wavetek to the desired 50 volts peak it was necessary to use the North-hills pulse transformer with a 1:5 winding. This transformer was designed to operate above $1 \mathrm{kHz}$, yet somewhat distorted the sawtooth waveform at a $2 \mathrm{kHz}$ fundamental frequency. See trace one in oscilloscope photograph, figure 12. This distortion was not considered serious and, when digitized and fast Fourier transformed, the waveform did indeed contain all the harmonic components of the fundamental sawtooth frequency, as expected. Figure 13 shows the results of this FFT as printed by the computer. The signal out of the wave generator was pulsed to provide the ability to selectively sample the diffracted signal all by itself without interference from extraneous signals reflected off of the endplates or other far-away structures. This pulsing technique greatly simplifies the analysis of the desired signal when the time of arrival of that signal is known precisely, and completely avoids sampling unwanted signals. In most cases, data were collected using one cycle of the sawtooth waveform. This one cycle was accomplished using gating signals sent to the Wavetek, Model 114, frequency generator from the Interface Technology, Model RS-648, timing simulator. 



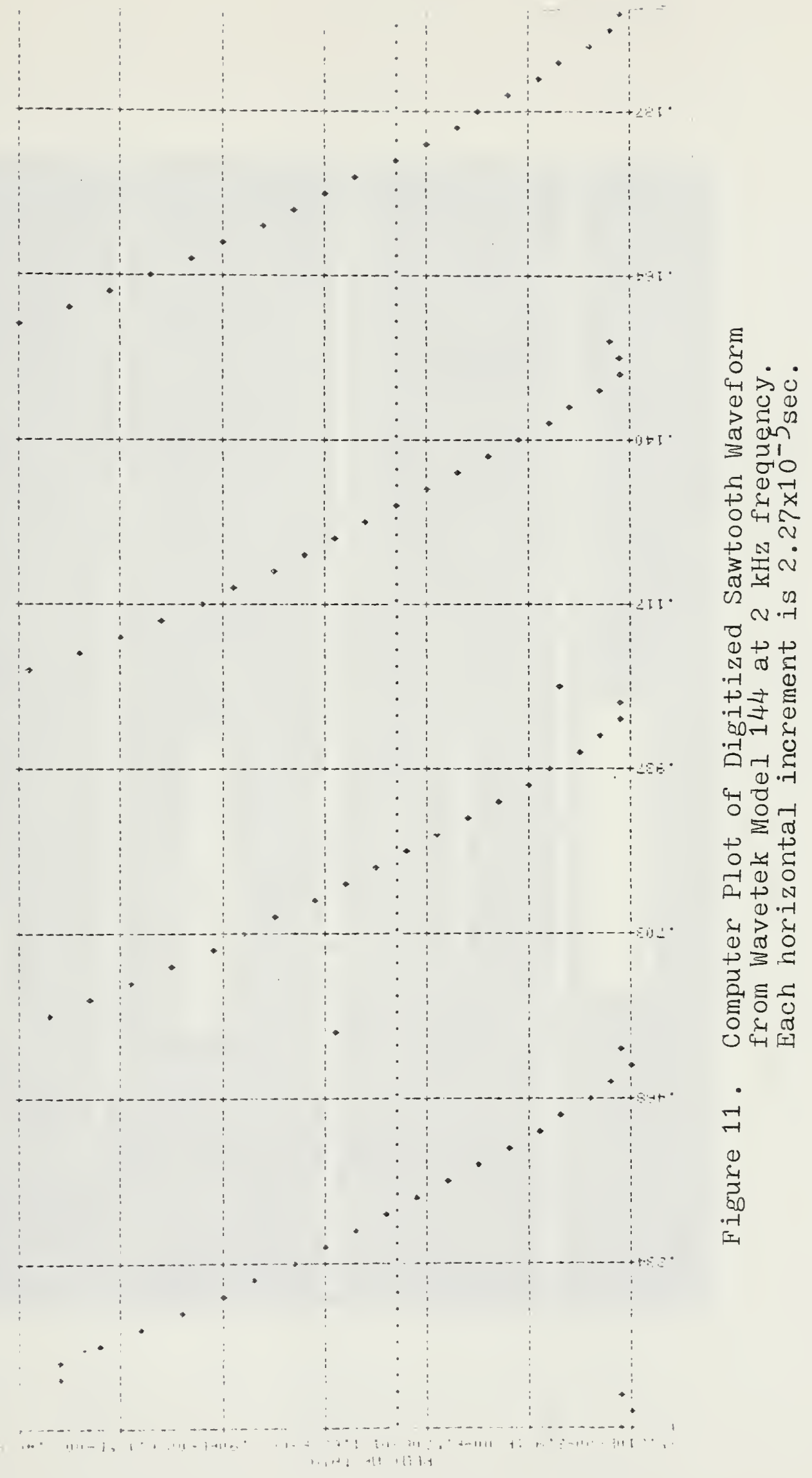





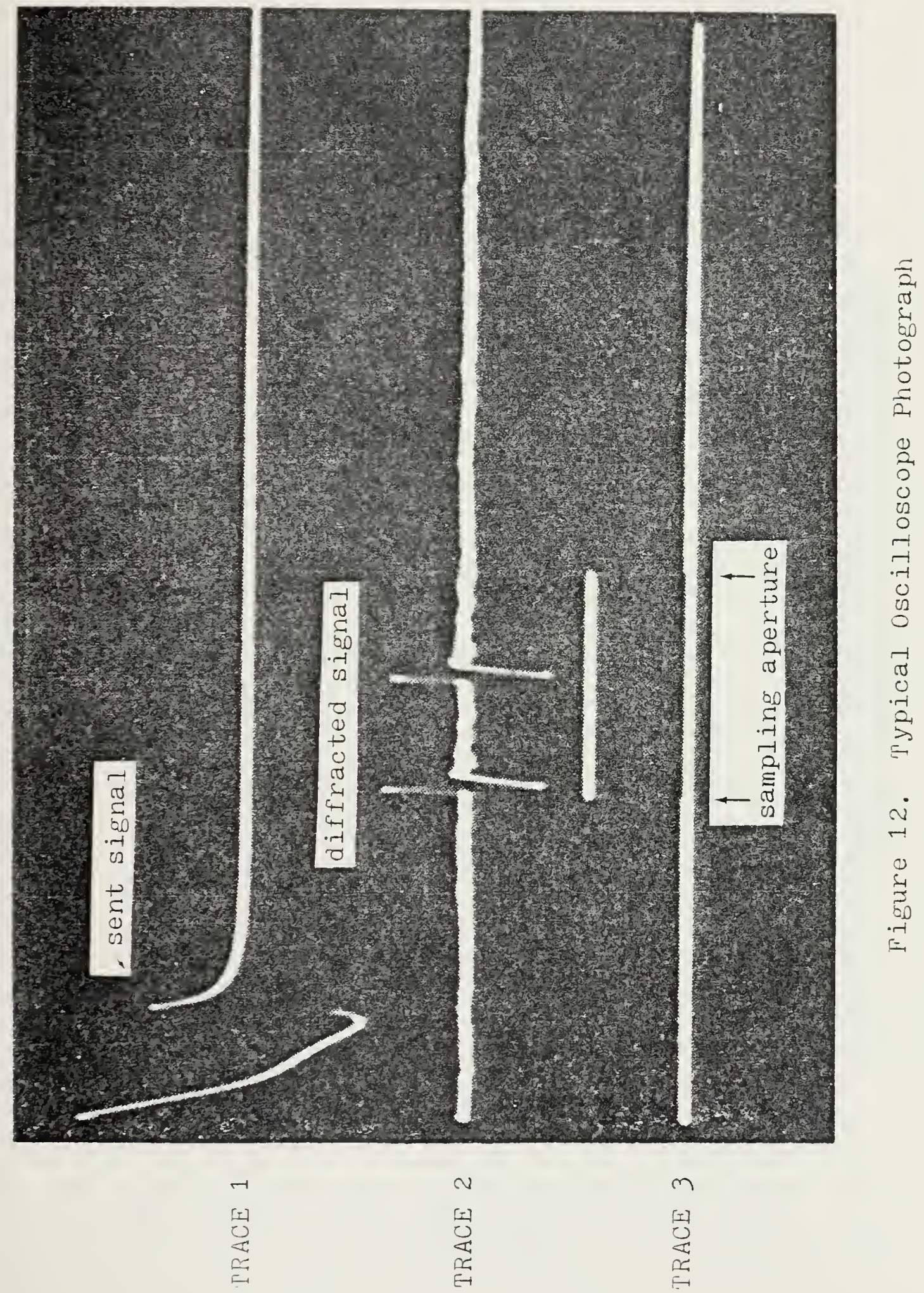





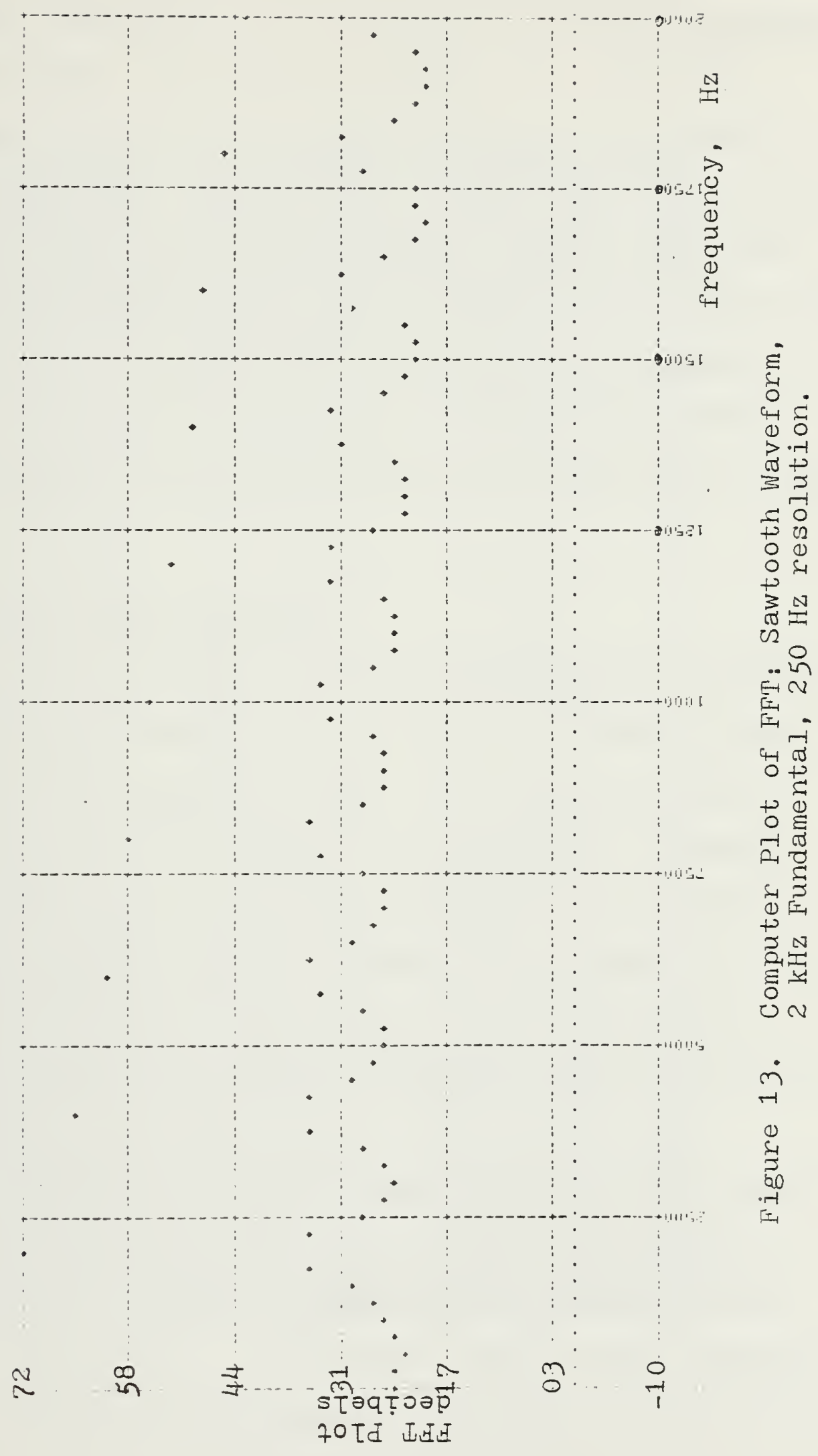



Although only one cycle of the sawtooth wave was generated electronically, two acoustical pulses propagated from the source. See trace 2, figure 12. The first acoustical pulse was the result of the first positivegoing voltage rise at the leading edge of the cycle, and the second acoustical pulse resulted from the positivegoing voltage spike at the trailing edge of the cycle. Thus, it was impossible to achieve less than two acoustical pulses, a situation not detrimental to the experiment. Figure 14 shows a series of pulses with a typical $10 \mathrm{msec}$ period.

\section{E. RECEIVED SIGNAL ANALYSIS}

The received signal was sent from the probe microphone to a low-noise voltage amplifier, the PAR Model 113 preamp, then to a high-pass filter, set to eliminate low frequency noise. From there it was sent to a low-pass filter, set to prevent aliasing effects in the signal sampling, then to another PAR Model 113 preamp, for a total system gain of $46 \mathrm{~dB}$. The analog output of the final voltage amplifier was sent to the Phoenix $A / D$ converter for digitizing

The diffracted energy was separated out of the analog signal and was analyzed by first quantizing the signal, converting it to digital values, and performing a fast Fourier transform on the digitized sample using software in the Interdata mini-computer. The output of the FFT was 


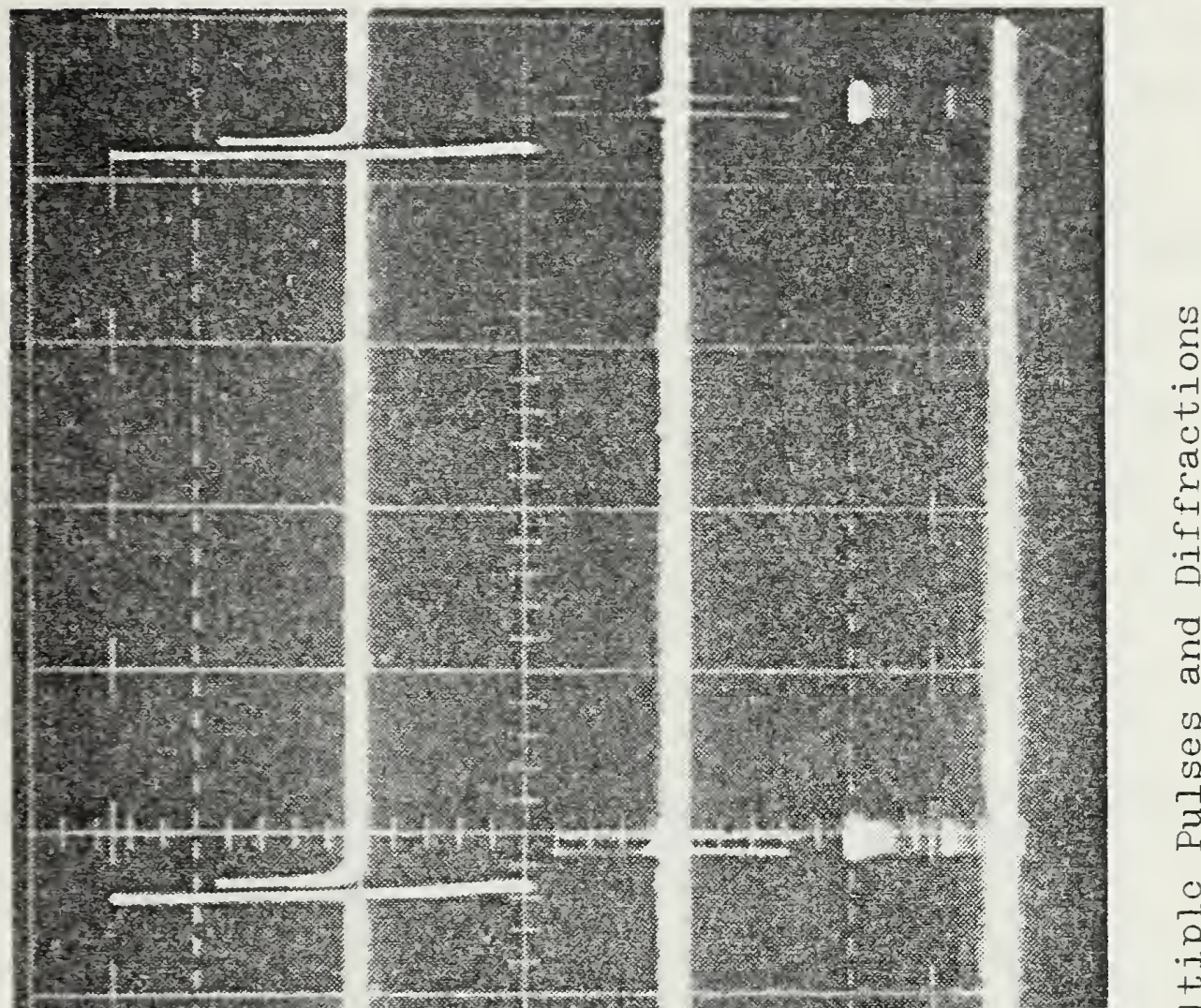

- 대

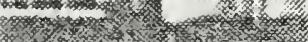

tit.

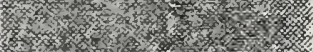

1.t.
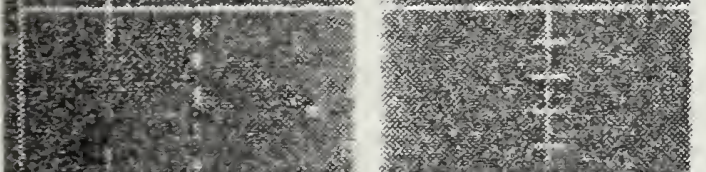

4her
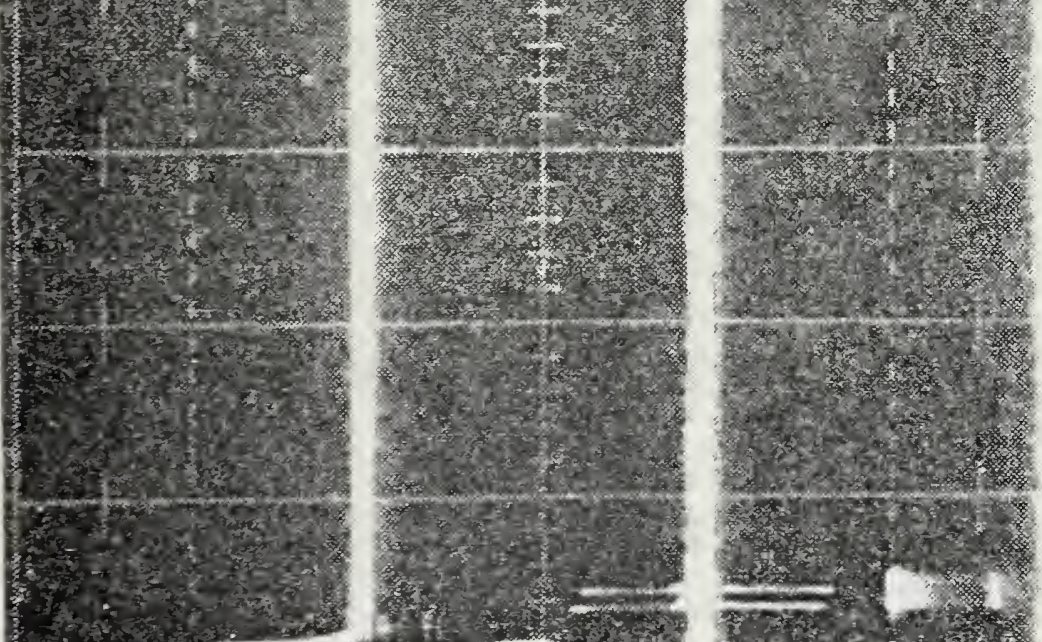

0

U2

孚

$-1$

प.

in

站

$\stackrel{-}{-1}$

0

30

50

is 

plotted and/or printed, displaying the amplitude and phase angle of the frequency components.

The separation of the diffracted signal was accomplished by precisely timing a sampling aperture. This was done by means of the Interface Technology timing simulator set to bracket the diffracted signal, and hence gate-out all unwanted signals. This was accomplished to 100 nanosecond accuracy. Within this sampling aperture the Wavetek Model 144 frequency generator was triggered from the timing simulator to produce rectangular pulses, each of which in turn triggered the Phoenix A/D converter to sample the analog signal. Each rectangular pulse corresponded to onehalf the period of the sampling frequency, as set on the Wavetek. Figure 15 shows a typical diffracted waveform with the rectangular sampling pulses superimposed.

The number of samples gathered in each sampling aperture was set as a power of two, i.e., 64, 128, 256, etc., as required by the FOURONE FFT algorithm. A number of factors had to be balanced carefully in order to process the data and obtain results for frequencies contained in the sawtooth waveform. The following formula was applicable,

$$
\frac{\text { sampling frequency }}{\text { number of samples }}=\text { frequency resolution. }
$$

The center frequency of each bin of resolution must be an exact multiple of the frequencies of interest, i.e., the harmonics of the sawtooth fundamental frequency. Also, by 







setting the sampling frequency as an integral multiple of the resolution, side lobes are avoided in the frequency domain. Since the number of samples taken must be a power of two then the sampling frequency, which, of course, must also satisfy the Nyquist criterion, must then satisfy the above formula. For example: if the fundamental frequency of the sawtooth signal was two kilohertz, providing every harmonic above that, then the frequency resolution must be a multiple, i.e., 500, 1000, 2000 Hertz. Now let the number of samples, taken within the sampling aperture, be 128, then the sampling frequency must be 256,000 Hertz for a resolution of 2000 Hertz.

Data for the experiment were taken at various fundamental frequencies, but normally either $2 \mathrm{kHz}$ or $2.5 \mathrm{kHz}$ was used. These required sampling frequencies of $256 \mathrm{kHz}$ and $320 \mathrm{kHz}$ respectively if the number of samples was kept constant at 128. It is important to note, that, the width of the sampling aperture is the limiting factor for the frequency resolution. In other words, if the received signal is 500 microseconds in duration, requiring a 500 microsecond aperture, then the resolution can be no less than $2 \mathrm{kHz}$. The wider the aperture, the better the resolution. However, when gating out unwanted signals, wide sampling apertures are not desired.

Due to the somewhat low source level of the signal and the relatively high self-noise of the receiver, it was important to improve the signal-to-noise ratio. One obvious 

place to do this was in the computer software. A computer program was written to average the digitized values over an ensemble of sampling apertures for as many as 9,999 blocks of data. In general, 1000 blocks, each of 500 microsecond duration, and each containing 128 samples were time averaged.

The improvement in the signal-to-noise ratio using this time averaging technique is $10 \log \mathrm{N}$, where $\mathrm{N}$ is the number of blocks averaged. Thus, the noise in the system, being random in nature, is averaged out, with an improvement being $30 \mathrm{~dB}$ in the signal-to-noise ratio for the 1000 block case. The results of this time-averaged ensemble of 128 samples were then fed into the FFT algorithm.

Once the results of the FFT were obtained, they were compared to direct transmission experiments with the barrier removed, providing essentially a transmission loss figure for the diffracted energy referenced to either direct-path transmission or reflection off a mirror surface at the barrier apex. 



\section{EXPERIMENTAL RESULTS}

A. DIFFRACTION IN THE FORWARD DIRECTION BY THE WEDGE

The diffracted pressure occuring in the forward direction, that is, propagating across the wedge away from the source and into the geometric shadow region, was by far the easiest to analyze. This diffracted pressure is compared to a mirror reflection occuring at the apex of the barrier, for every case.

With the source positioned at $\theta_{0}=15^{\circ}$, and $r_{0}=25 \mathrm{~cm}$, measurements of the acoustic field in this forward direction were taken with the receiver positioned at every $15^{\circ}$ angle, from the side of the wedge opposite the source, $\theta=270^{\circ}$, up to $\theta=195^{\circ}$, the edge of the shadow region. The radial distance of the receiver from the apex of the wedge was also $25 \mathrm{~cm}$. The results of these measurements are better described graphically. Figures 16 through 20 present the results for each angle as a function of frequency. Also plotted on each graph is the Biot-Tolstoy theory as transformed by Medwin [Ref. 7 ]. As can be seen, the experimental results agree with the theory to within one decibel, except at the very high frequencies where the receiver response had falien off sharply and the signal-to-noise ratio was degraded.

Figures 21 through 24 are plots of the data obtained from experiments run with the source now positioned at $30^{\circ}$ 



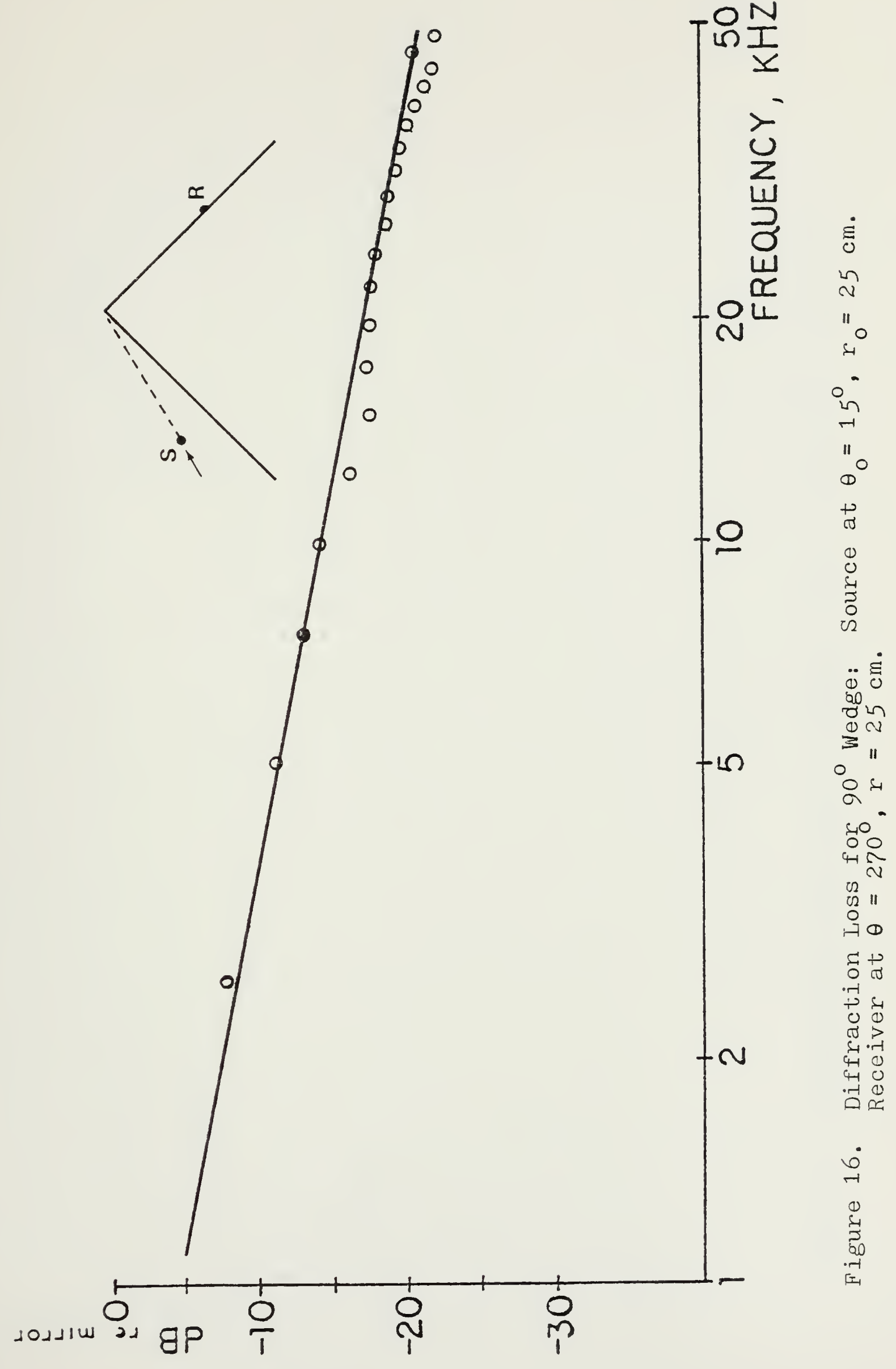





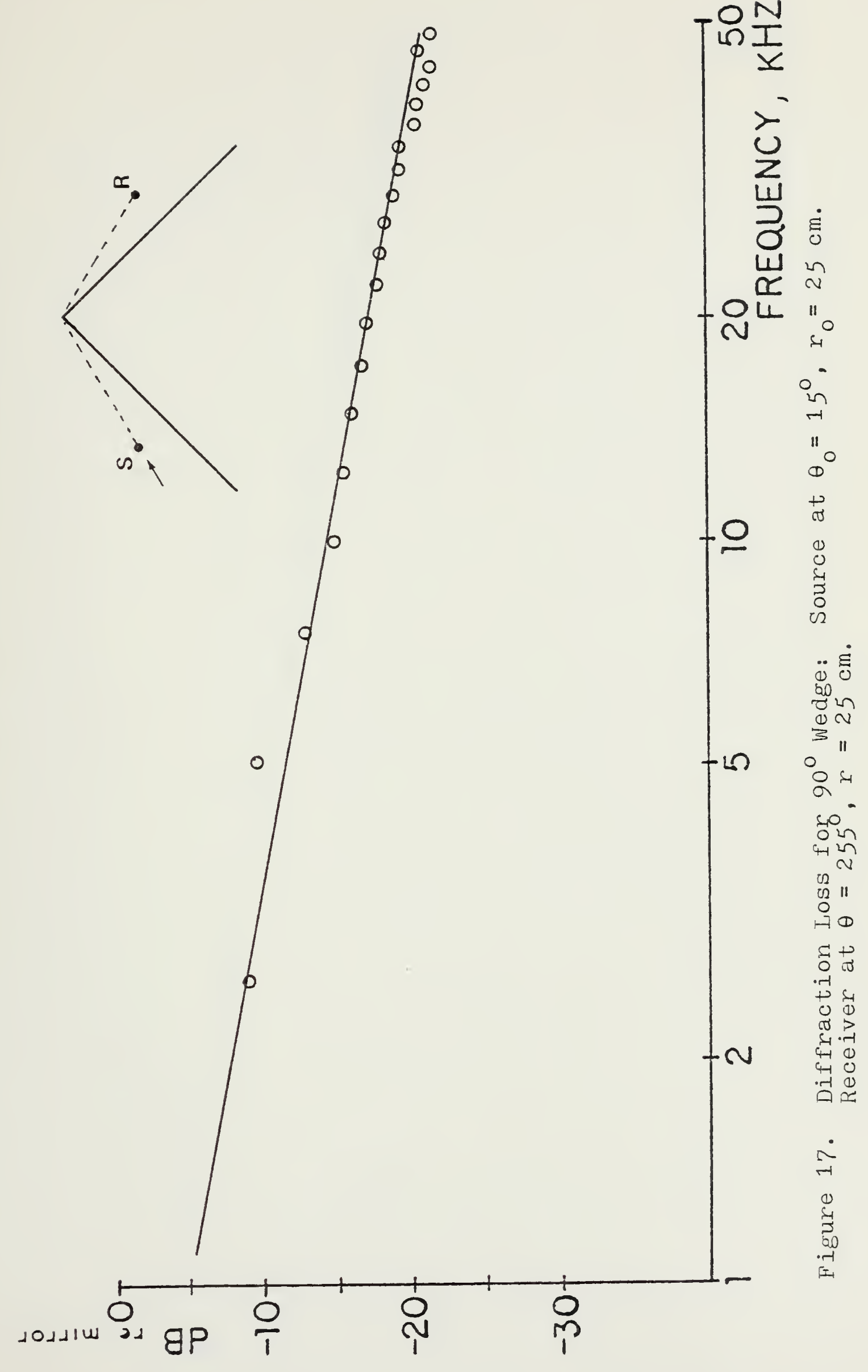





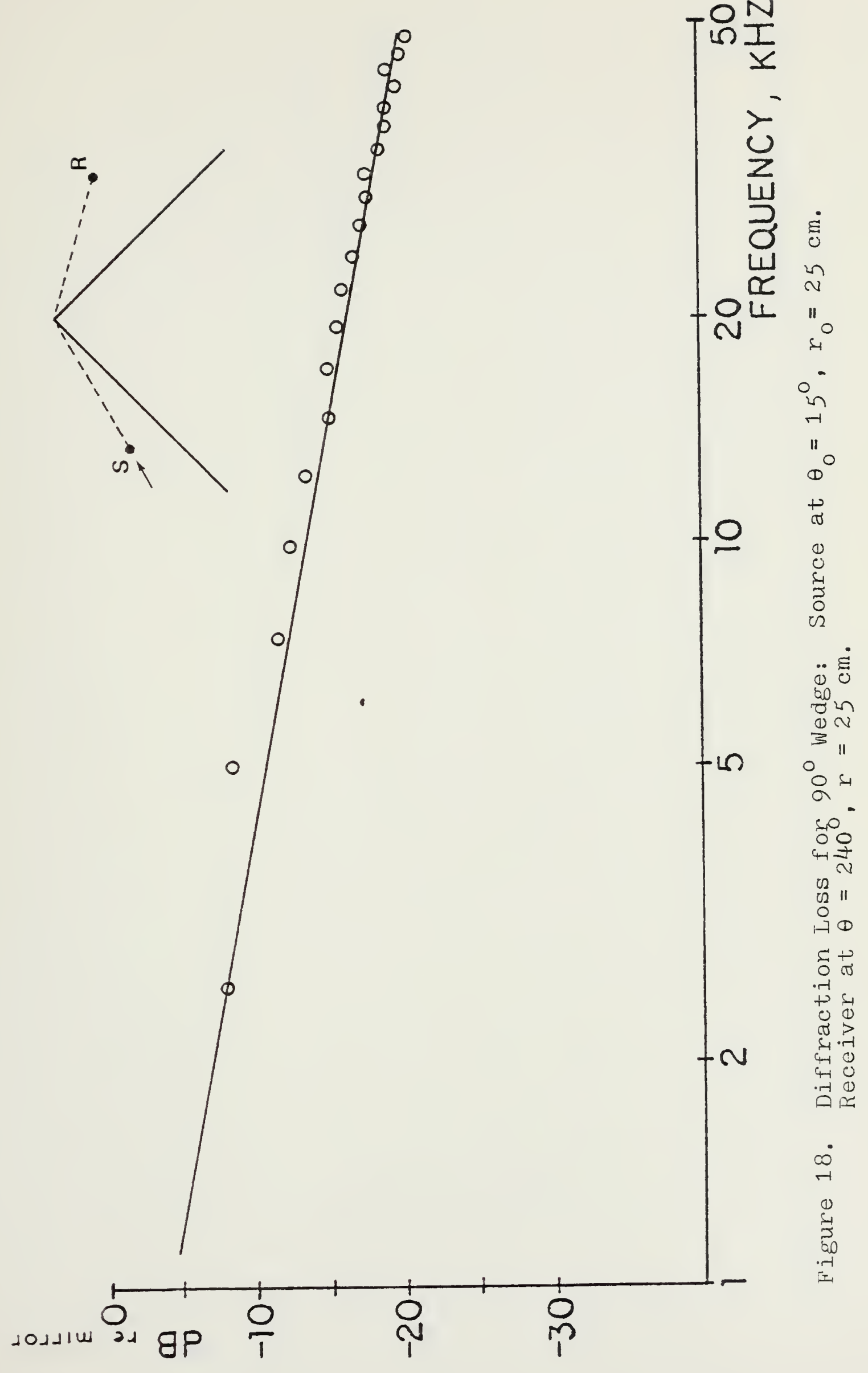





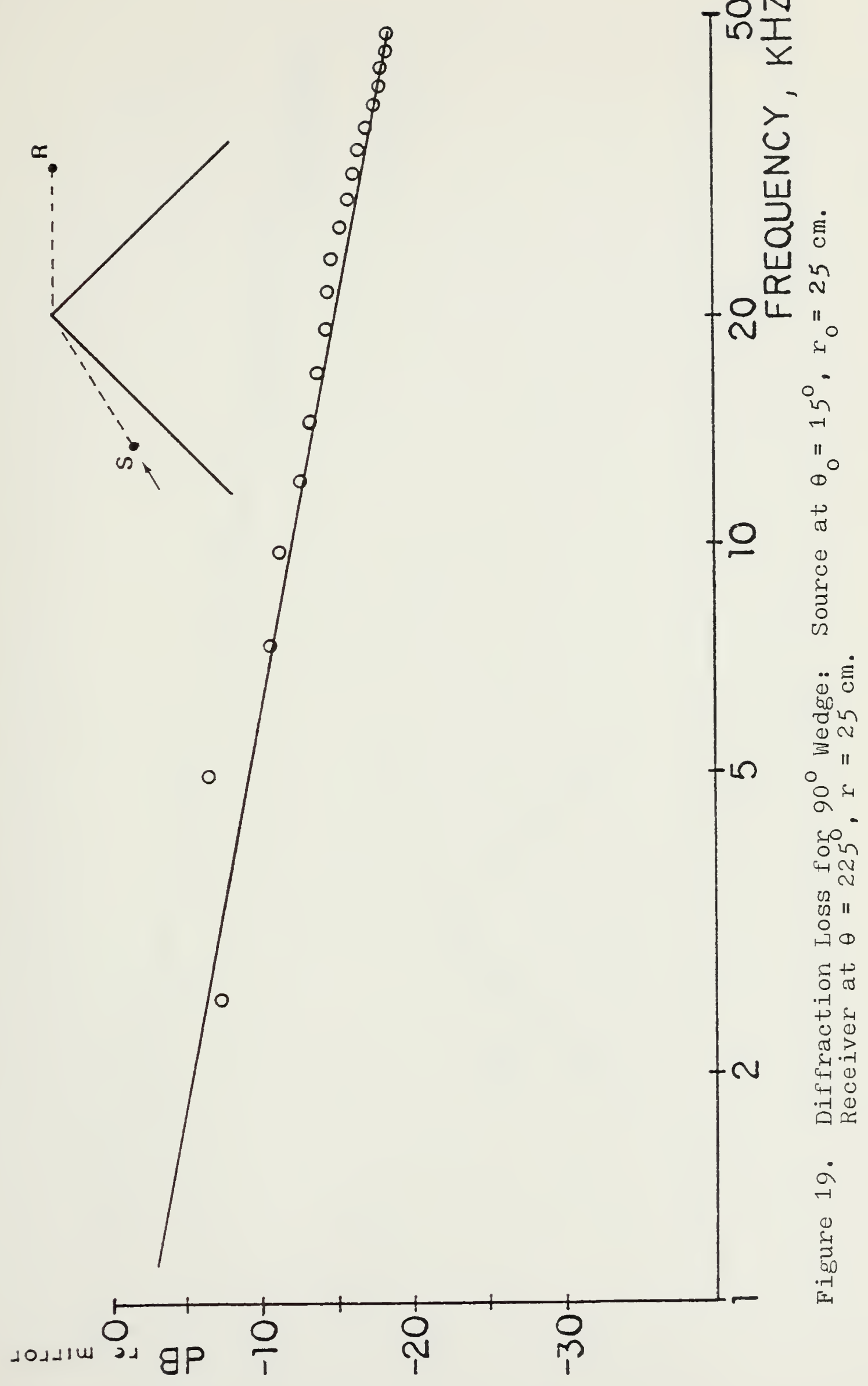





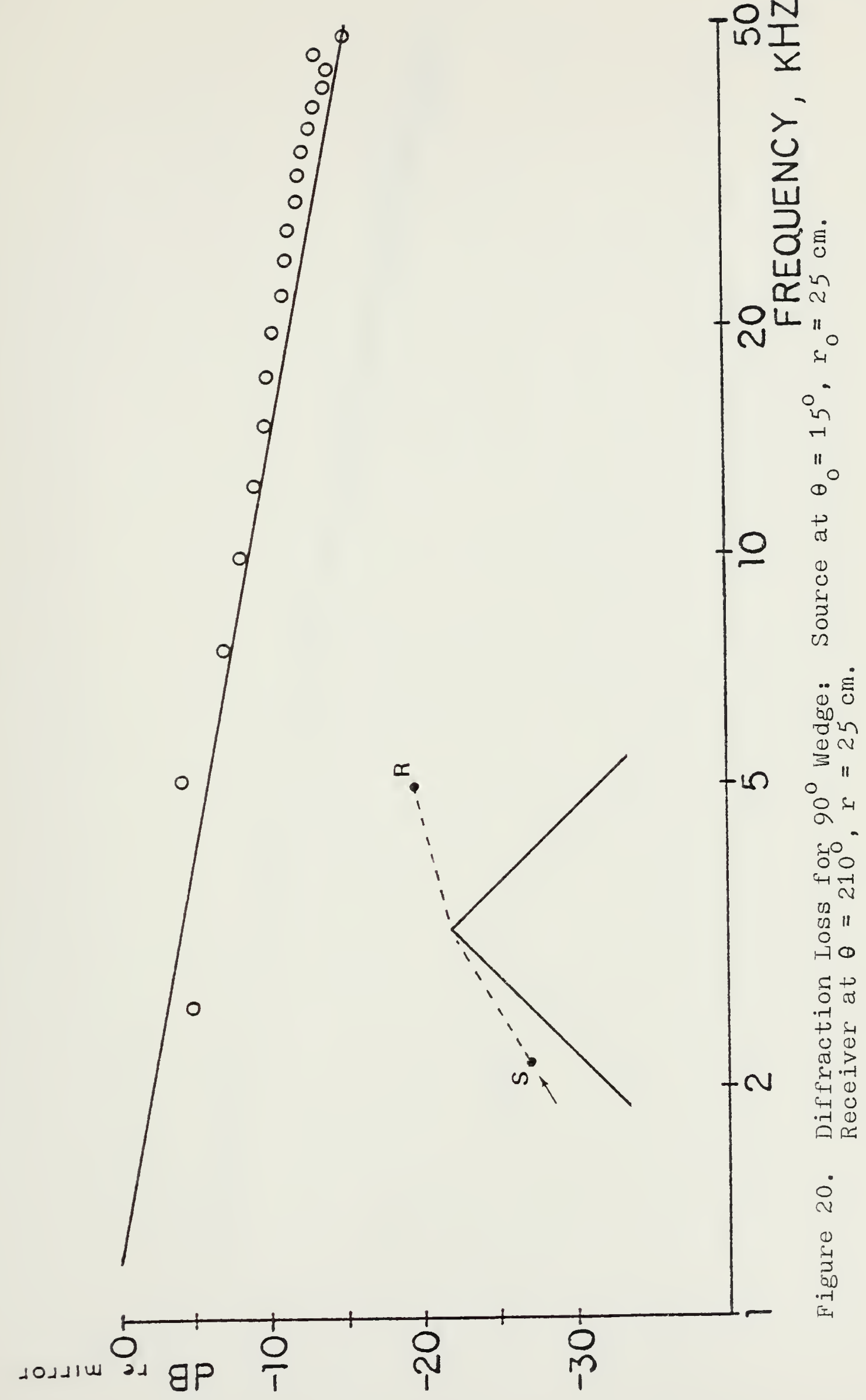





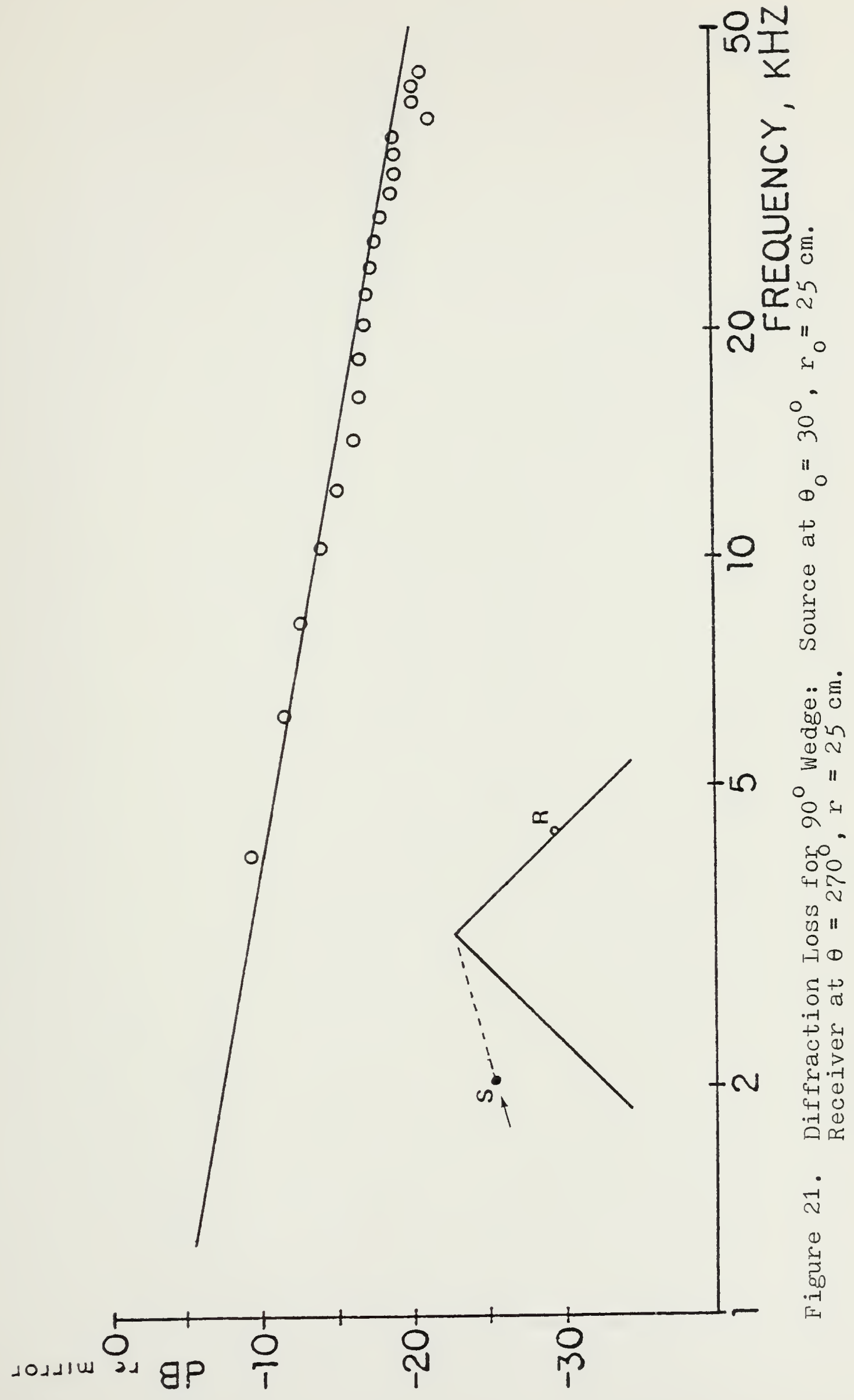





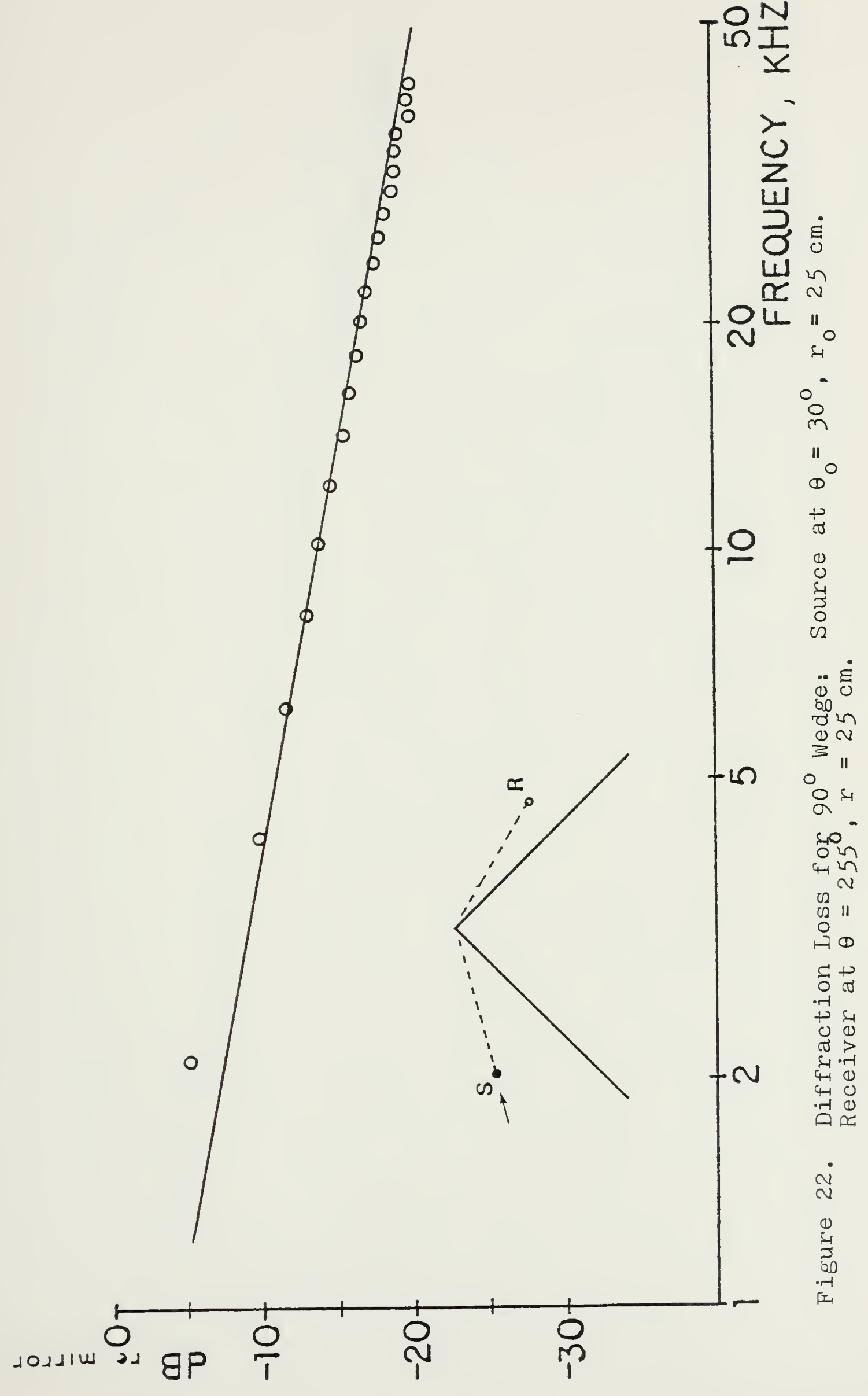





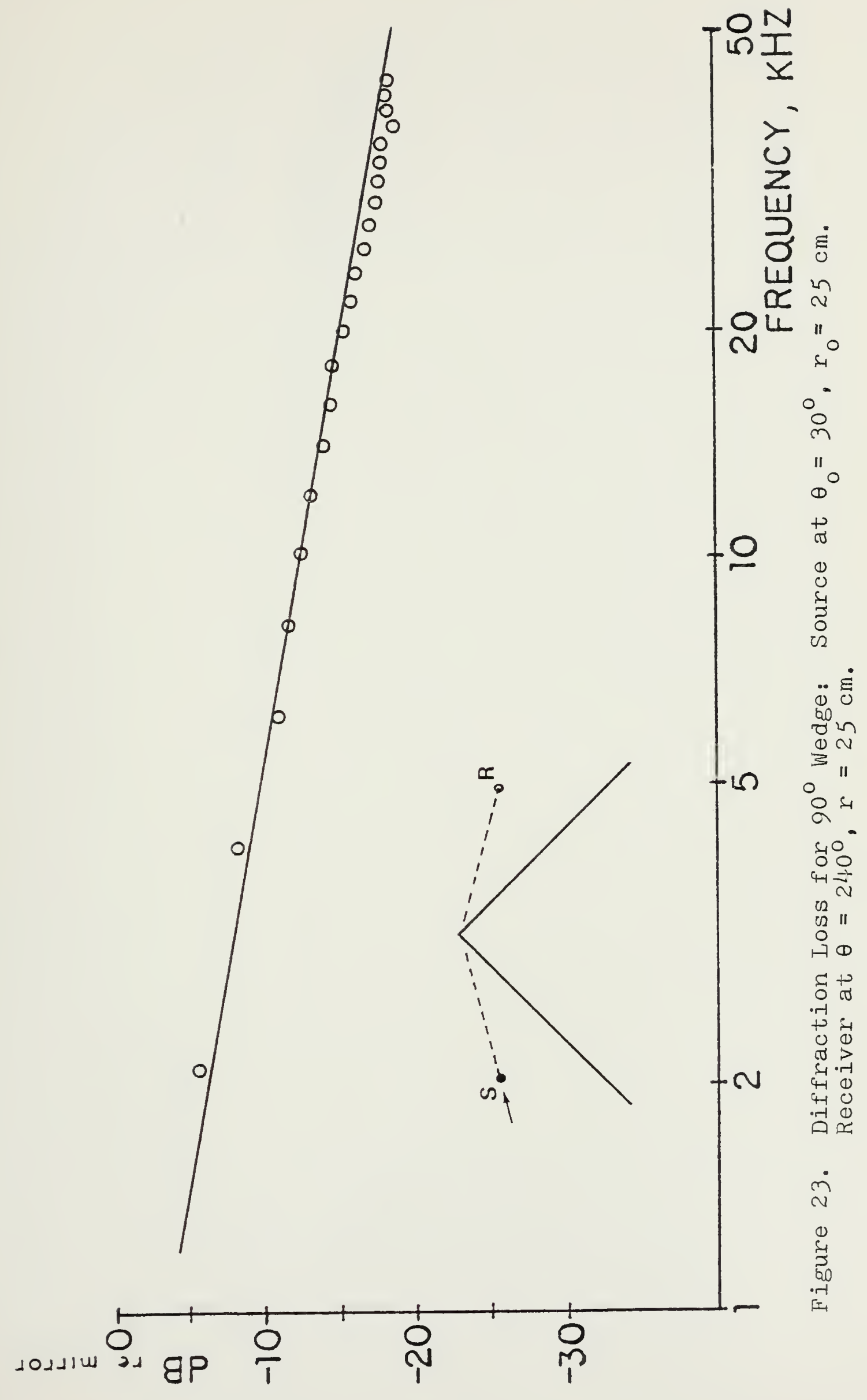





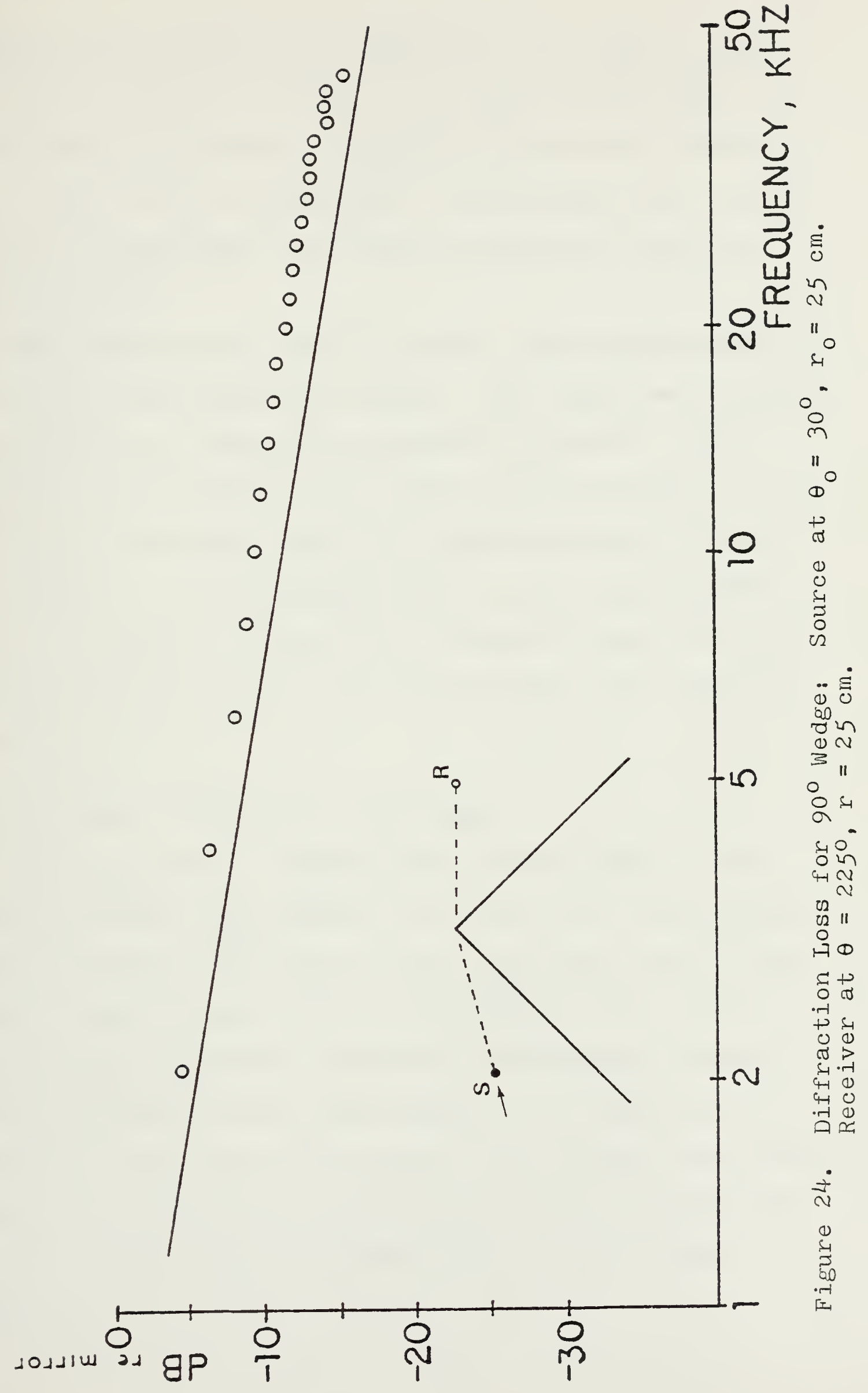



from the front face of the wedge, i.e., $\theta_{0}=30^{\circ}$. Again, the receiver was moved from the far face of the wedge, the side opposite the source, up to the "illuminated" region at $\theta=225^{\circ}$, in $15^{\circ}$ increments. In all cases, the source and the receiver were positioned at constant radial distance of $25 \mathrm{~cm}$.

One more special case for the $90^{\circ}$ wedge diffraction in the forward direction was studied. This experiment allowed for comparison to results published by Jonasson (1972). Jonasson positioned his source and receiver symmetrically $20 \mathrm{~cm}$ below the wedge apex at a radial distance of $32 \mathrm{~cm}$. The results of this experiment are plotted and compared to that of Jonasson in figure 25. Jonasson's theory is also plotted for comparison to the Biot-Tolstoy theory and to the data.

B. BACKWARD DIFERACTION BY THE WEDGE

The experiment is somewhat more complicated in the situation where the backward diffraction is to be studied. Here the source and receiver were on the same side of the wedge. The task was to study that energy which travels forward from the source, diffracts off the apex and then travels backward toward the receiver. The complication arises in that there also exists in this region the acoustic energy traveling directly from the source to the receiver, as well as the energy reflected off the plate toward the receiver. 



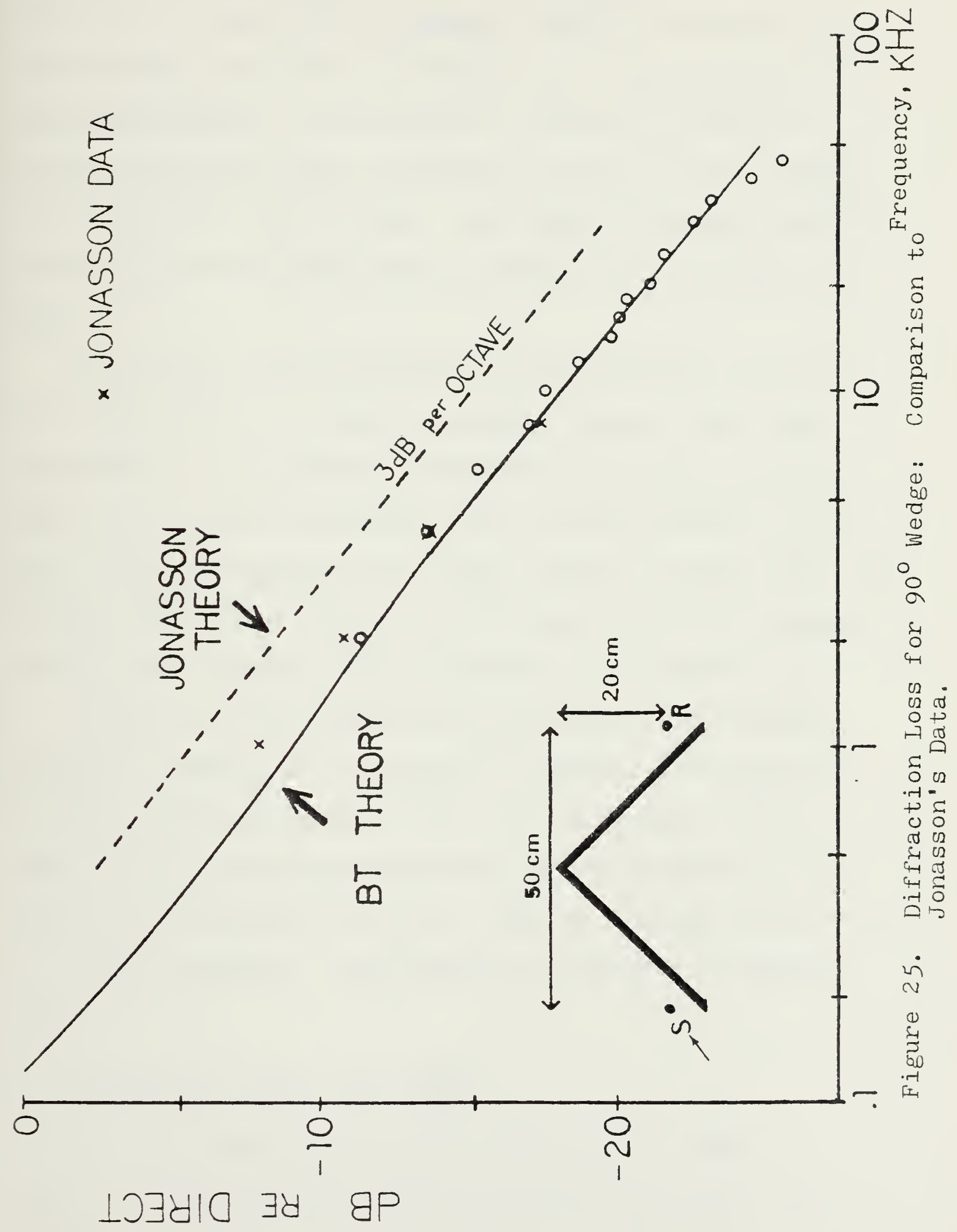



The diffracted signal can be successfully separated from the direct and reflected signals if the pulsing technique is used and the unwanted signals are gated out, as described previously in section IV. The results of these experiments are presented in figures 26 through 33, for the cases where the source is $15^{\circ}$ and $30^{\circ}$ above the front face of the weage. The radial distances for the source and receiver were kept constant at $25 \mathrm{~cm}$ from the apex.

Once again, the experimental results agree very well with the theory. It might be noted, however, that the agreement in the backward direction is not quite as good as in the forward direction. This is attributable to the fact that the backward diffracted energy is weaker than the forward diffracted signal by $25 \mathrm{~dB}$ and thus more susceptible to interference from self-noise in the system.

Insight into the behavior of the diffracted energy around the wedge can be gained by plotting this energy as a polar radiation pattern about the wedge apex. This is done for the two source positions at representative frequencies in figures 34 and 35 . Data is plotted for $\mathrm{kr}=46$. , or $10 \mathrm{kHz}$ frequency. The theory is from work by Medwin [Ref. 7].

C. DIFFRACTION ABOVE THE WEDGE

As can be seen in the polar plots on the previous pages, no experimental data was obtained in a 120 degree 



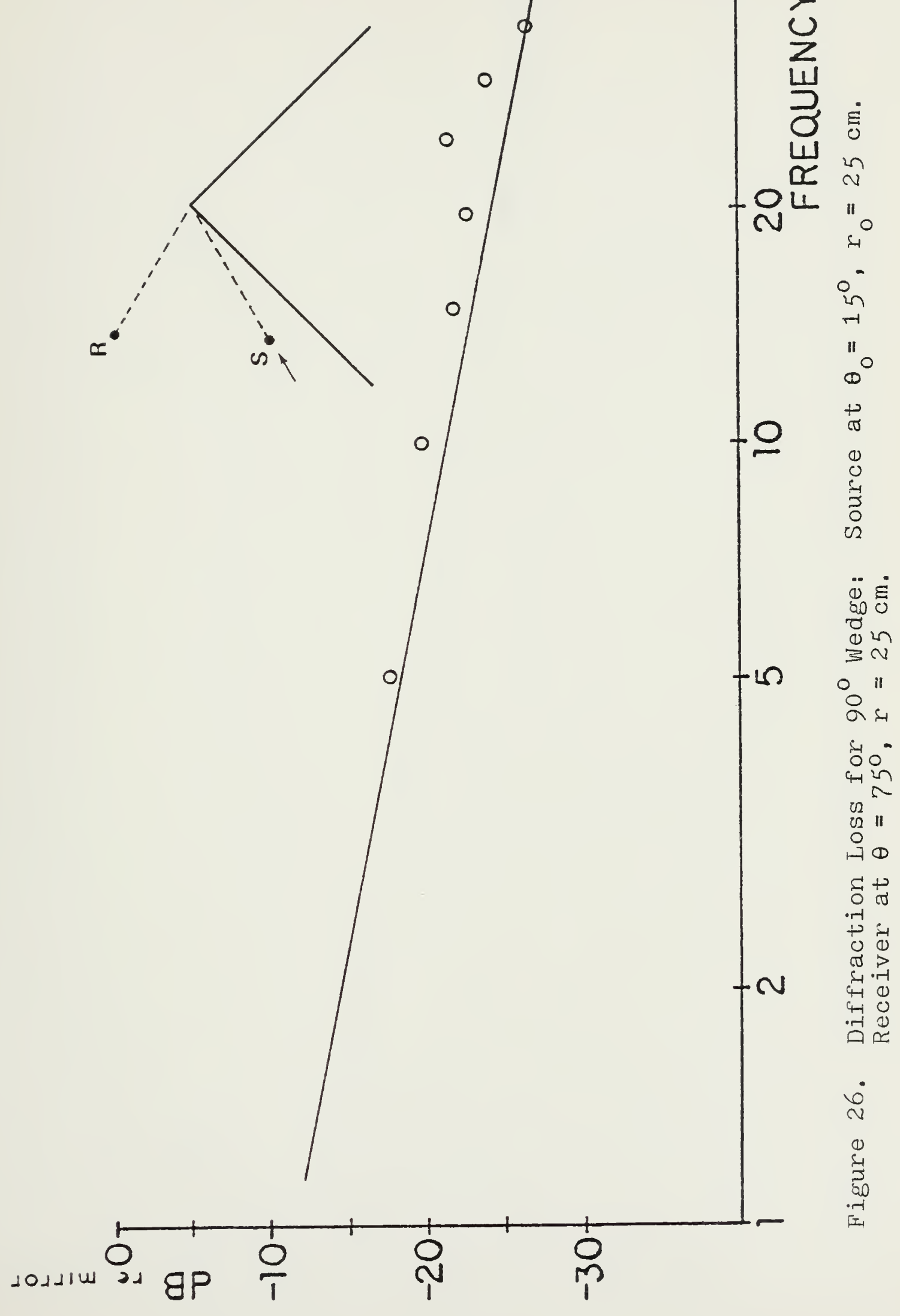





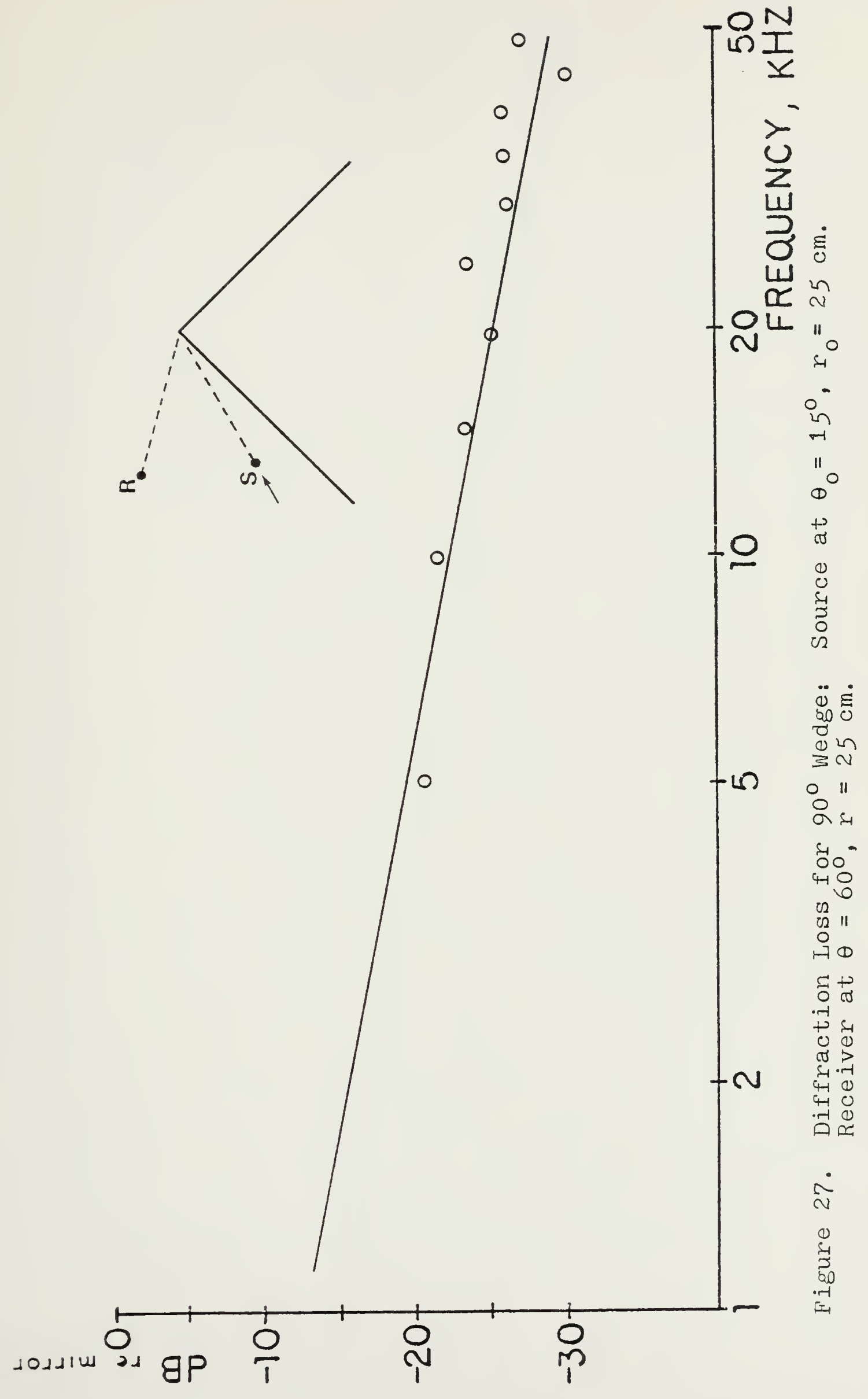





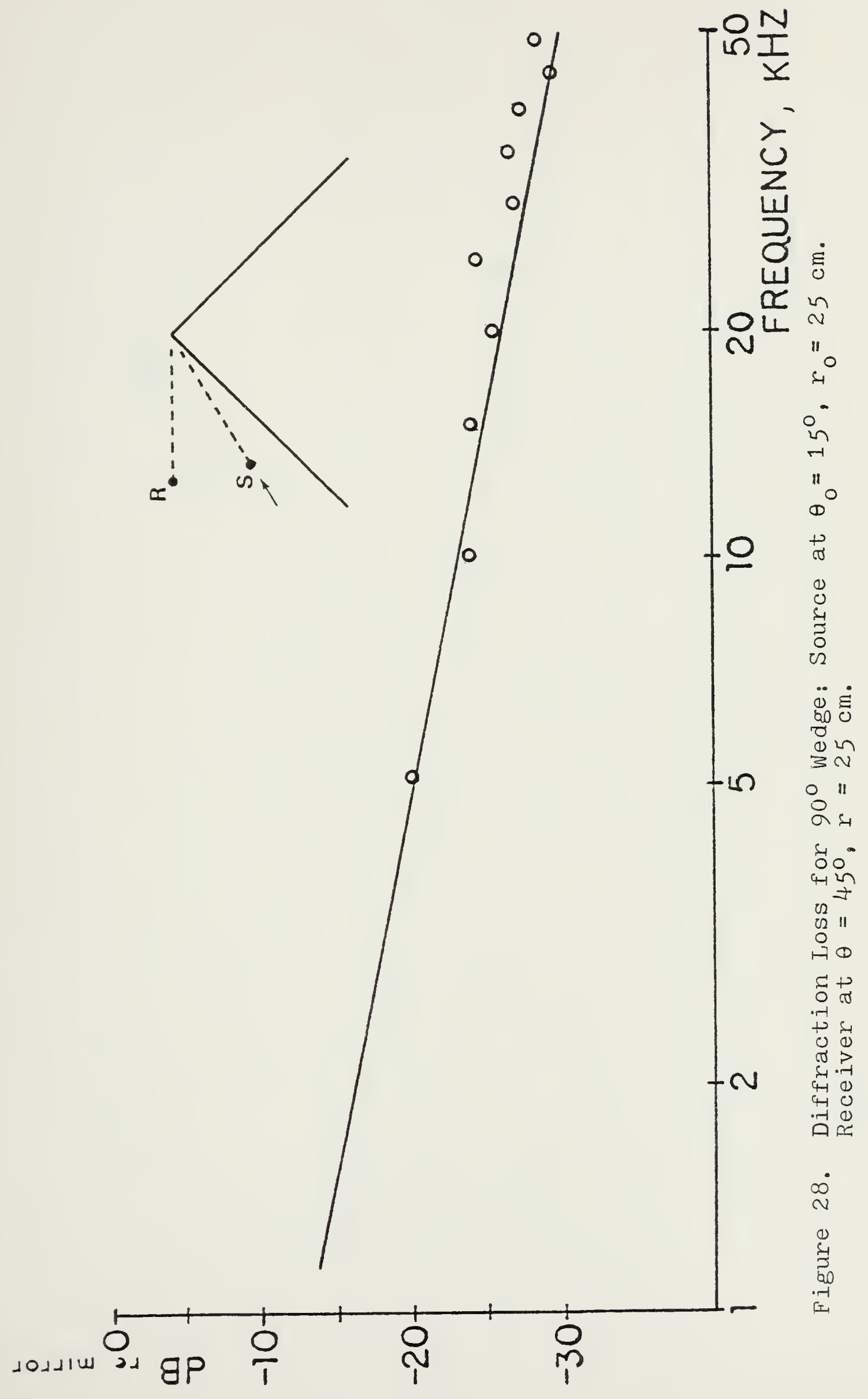





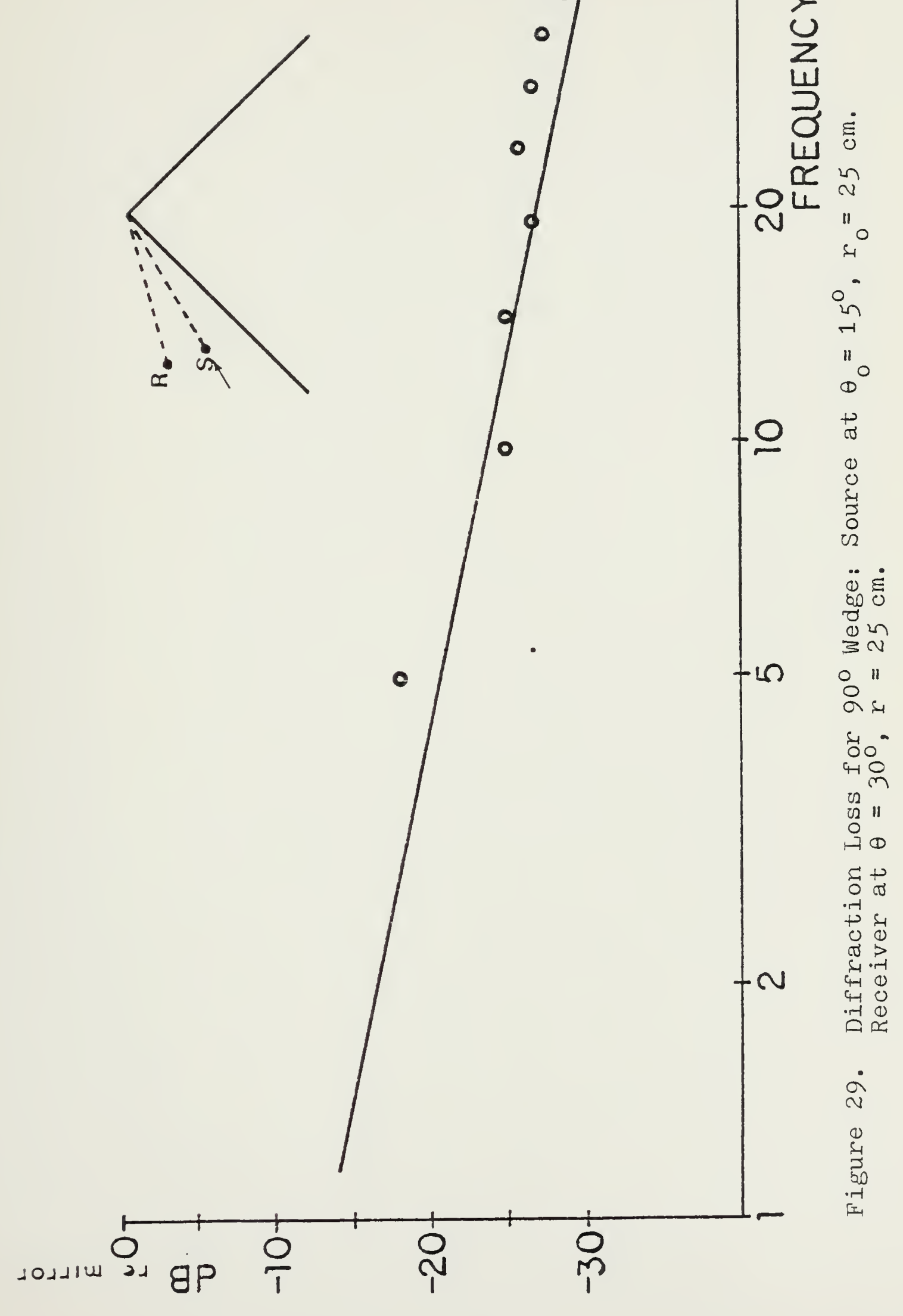





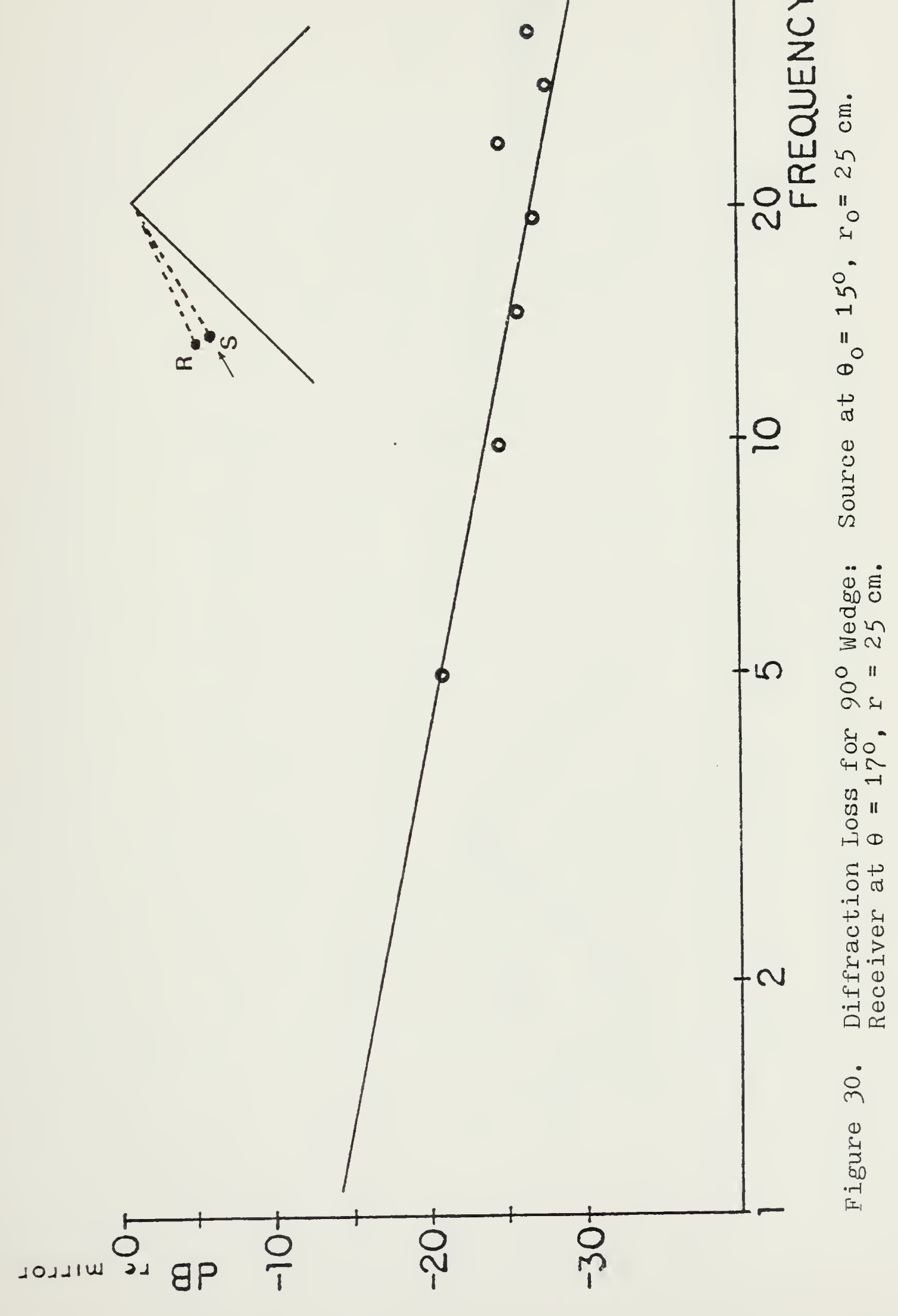





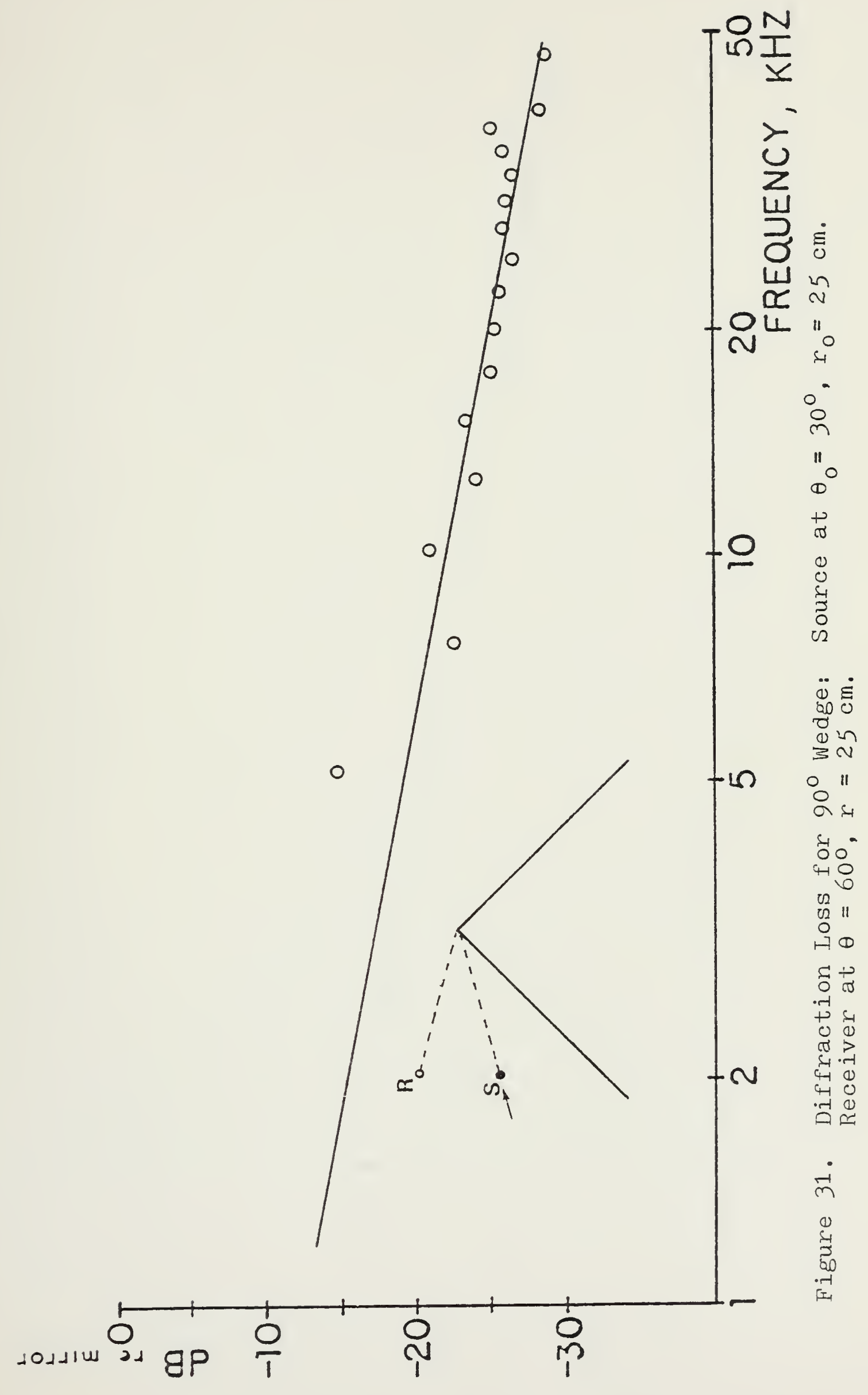





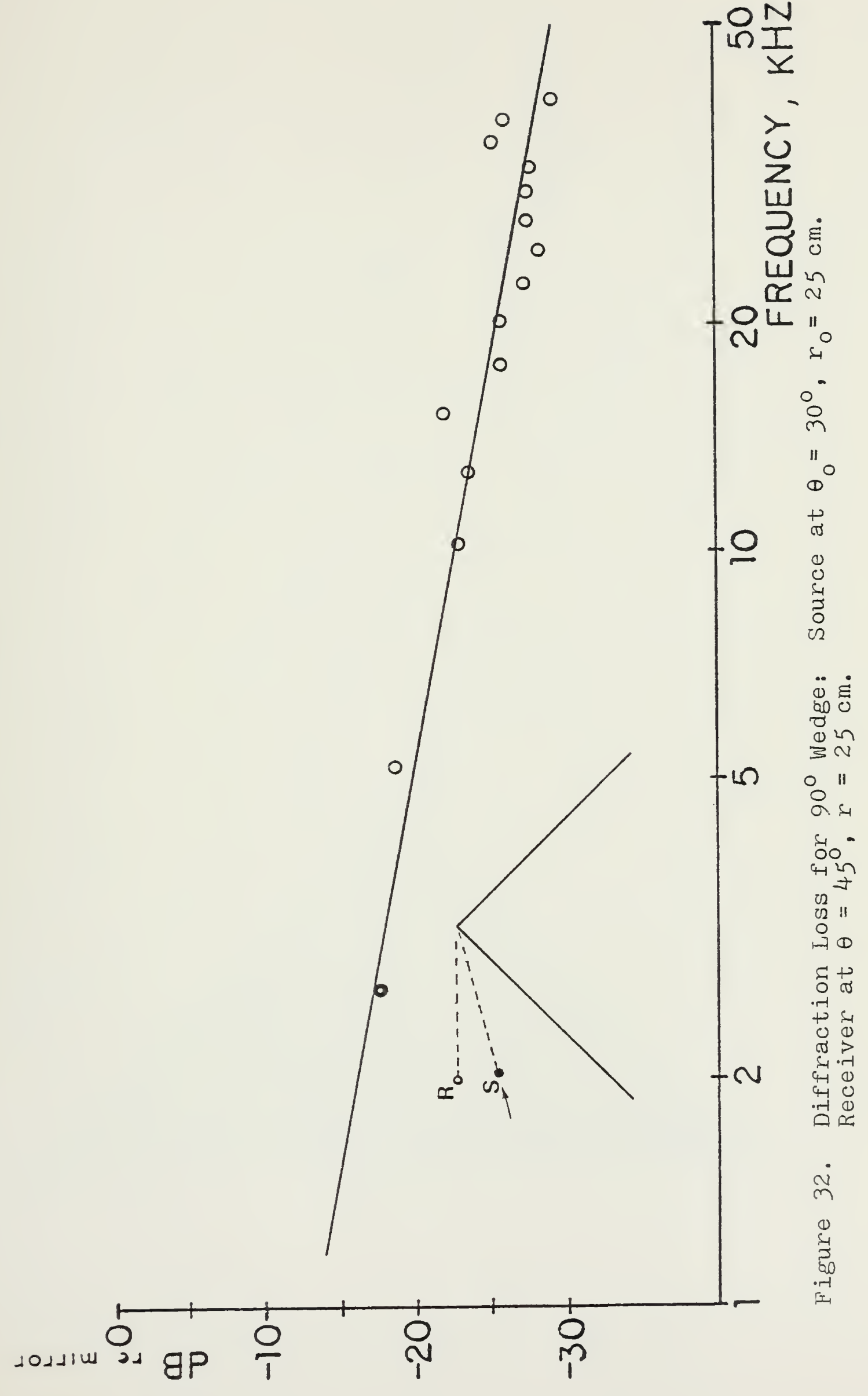




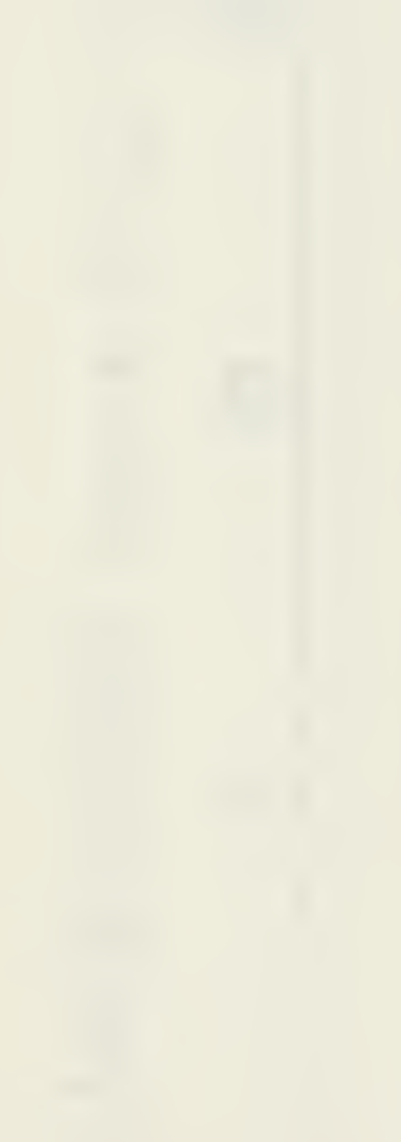




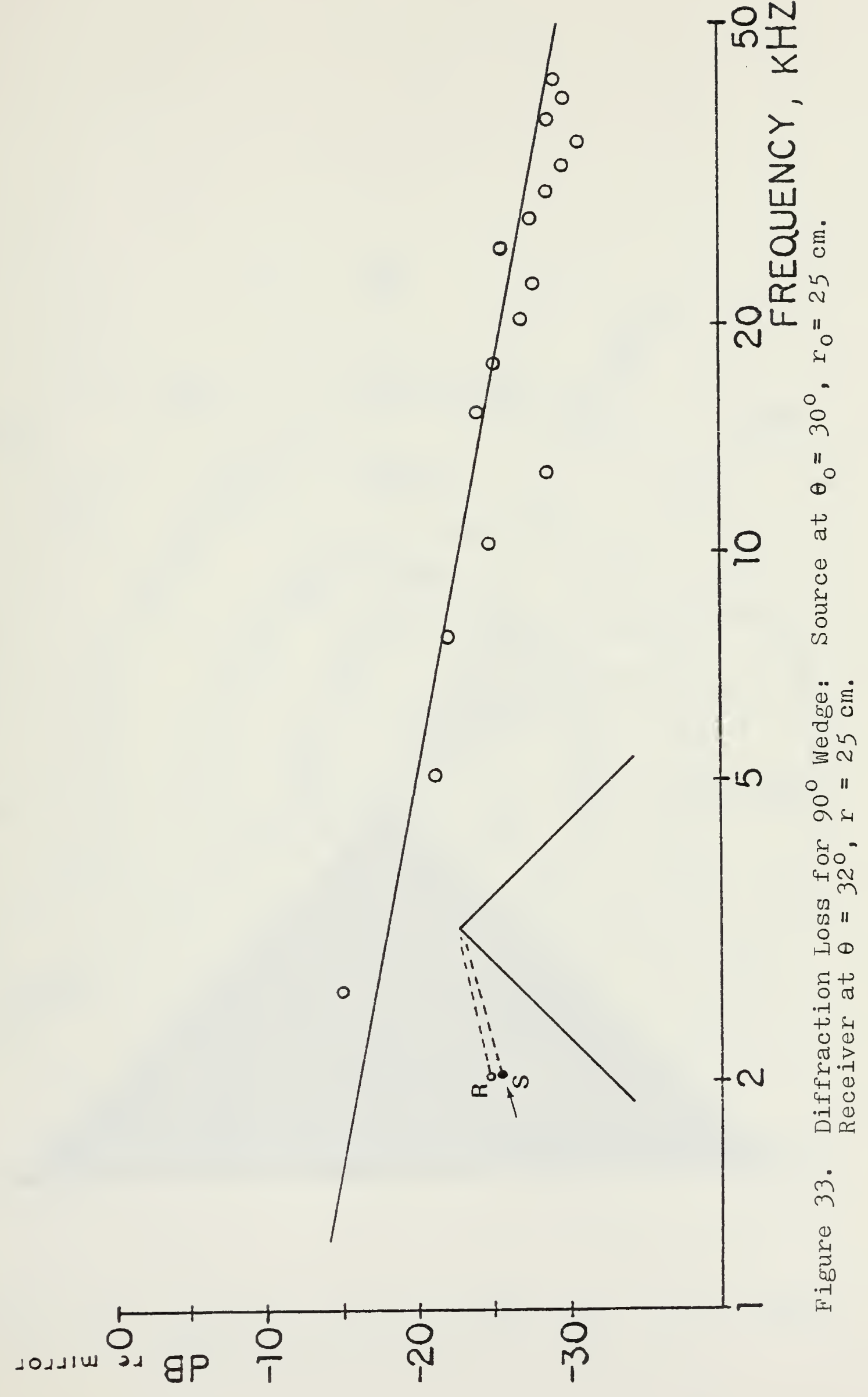





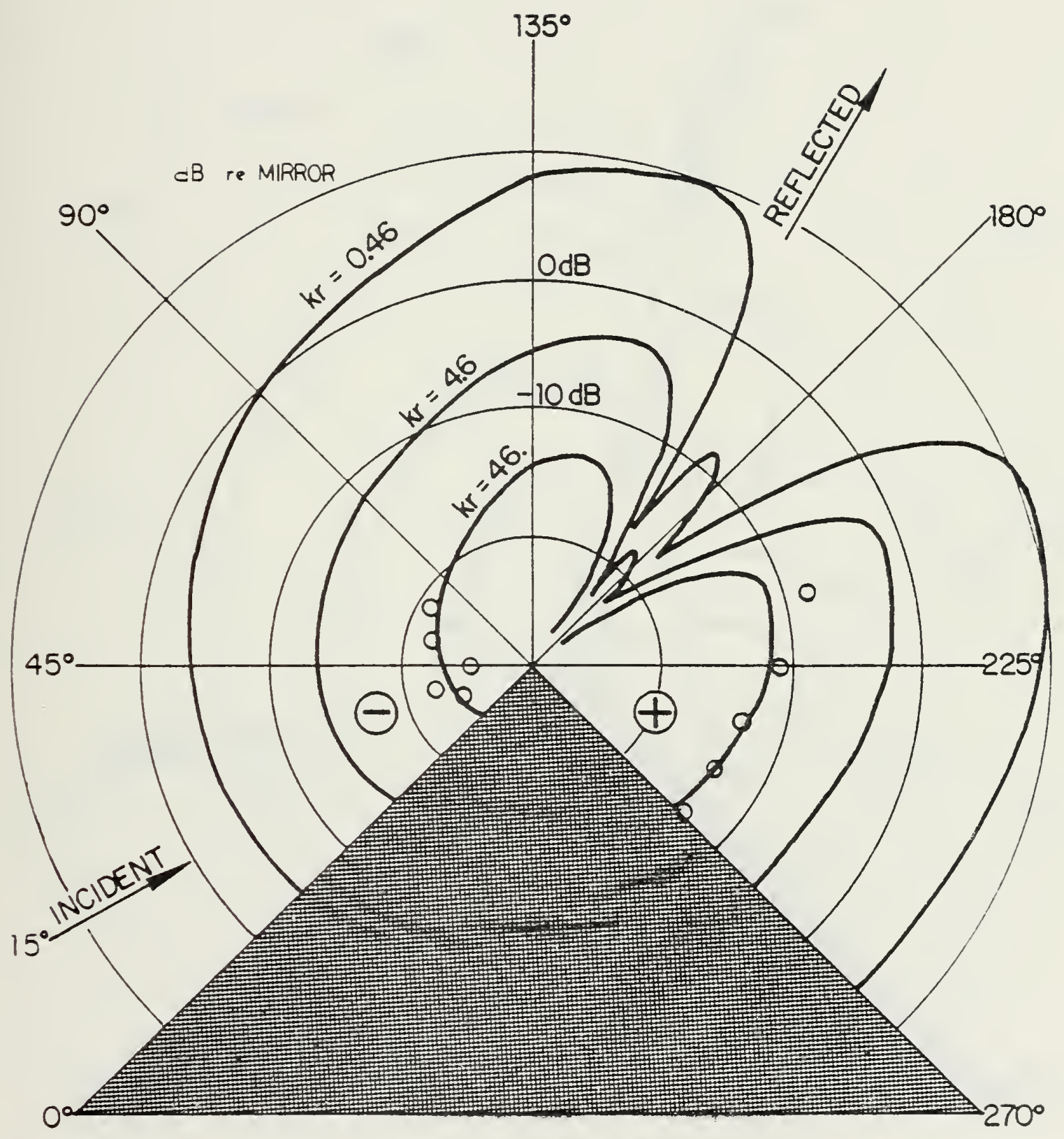

Figure 34. Polar Plot of Diffraction Loss for 900 Wedge. Source at $\theta_{0}=15^{\circ}, r_{0}=25 \mathrm{~cm}$. 



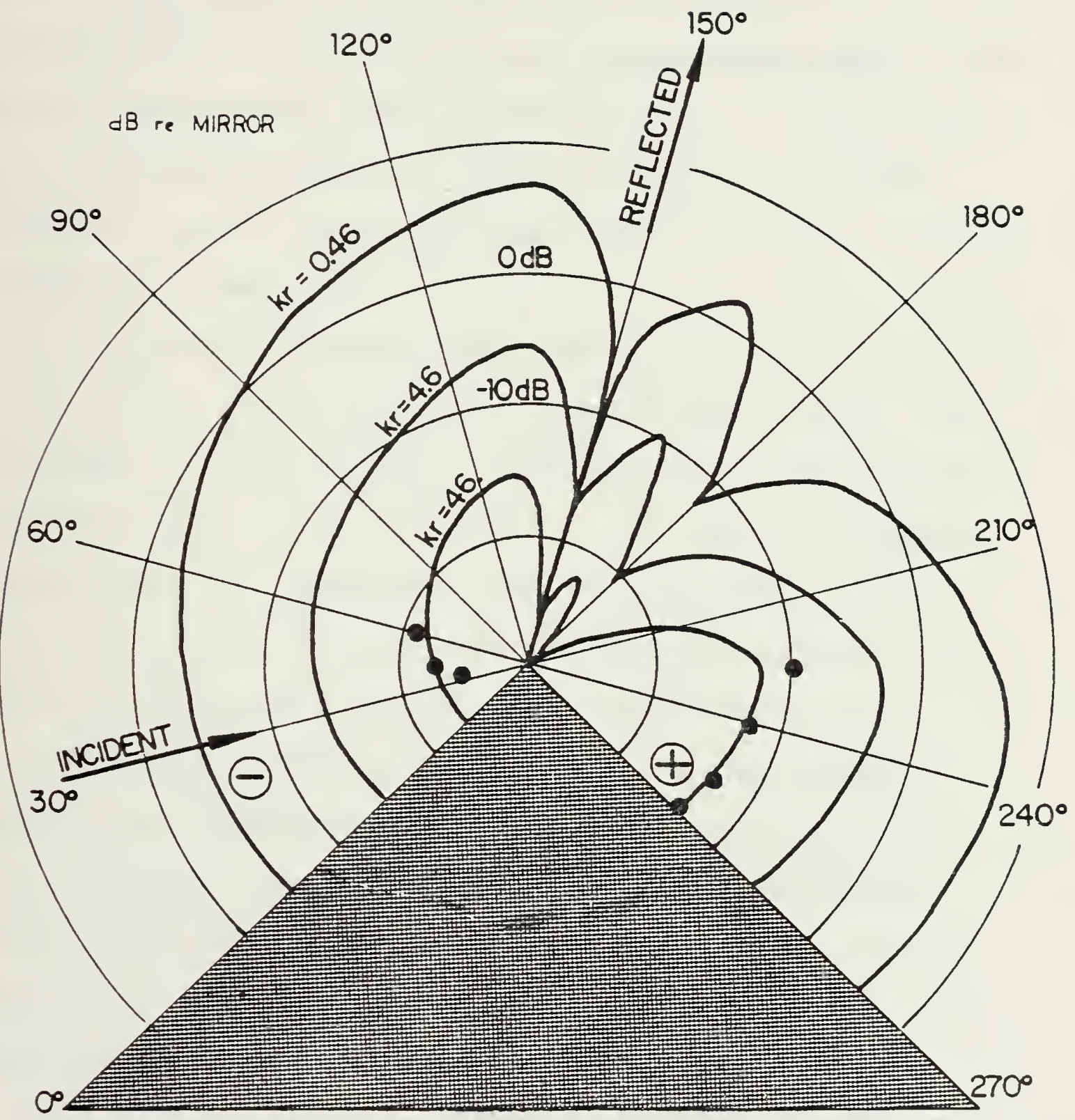

Figure 35. Polar Plot of Diffraction Loss for $90^{\circ}$ Wedge. Source at $\theta_{0}=30^{\circ}, r_{0}=25 \mathrm{~cm}$. 

region above the wedge. The reason for the lack of data in this region is because of the coexistence of the diffracted energy with the direct and reflected signals. The path-lengths of these three signals are nearly equivalent in this region, thus they form a compound signal which is impossible to separate by time gating techniques. However, attempts were made to deconvolve this superposition of the diffracted signal with the direct and reflected signals to obtain the diffracted energy by itself. These attempts are described below.

The sampling aperture was 'widened to bracket the entire signal pulse, where the direct signal came in first, then overlapped by the reflected, and finally followed closely, or overlapped, by the diffracted. The number of samples taken within the aperture, as well as the width of the sampling aperture itself, were increased by powers of two in order to maintain the integrity of equation 41 .

Once these samples were digitized, time-averaged, and fast Fourier transformed, the real and imaginary parts of this transform were stored on floppy disc. Similarly, results from experiments with the wedge removed, wherein just the direct signal was digitized, time-averaged, and transformed, were also stored in floppy disc.

The task then was to operate on the signal obtained without the wedge so as to derive, within the computer, signals identical in phase and amplitude to the direct and reflected signals of the wedge problem. These newly derived 

signals were then subtracted from the compound signal over the wedge leaving just the real and imaginary parts of the diffracted signal. This was done in the complex domain in order to maintain the phase integrity of the signals. To amplify; the output of the signal acquired with the wedge removed, was converted in the computer from the rectangular complex form into polar form, i.e.,

$$
a+j b=R e^{j \phi}
$$

where

$$
R=\text { amplitude of signal }=\left(a^{2}+b^{2}\right)^{\frac{1}{2}}
$$

and

$$
\phi=\text { phase of signal }=\operatorname{Tan}^{-1}\left(\frac{b}{a}\right) \text {. }
$$

The phase of the signal was then adjusted to correspond to phase shifts due to the difference in path length between the direct and reflected wedge signals and the signal without the wedge. The phase change for the direct signal is given by

$$
\Delta \phi_{\mathrm{d}}=\mathrm{k} \Delta \mathrm{r}_{\mathrm{d}}
$$

where

$$
k=\frac{\omega}{c}=\frac{2 \pi f}{c}
$$





$$
\begin{aligned}
& f=\text { frequency in Hertz } \\
& c=\text { speed of sound }
\end{aligned}
$$

and where

$$
\begin{gathered}
\Delta r_{\mathrm{d}}=r+r_{0}-r_{\mathrm{d}} \\
r=\text { distance from apex to receiver } \\
r_{0}=\text { distance from source to apex } \\
r_{\mathrm{d}}=\text { distance traveled by the direct signal }
\end{gathered}
$$

The quantity $r_{d}$ can be formed from the law of cosines where

$$
r_{d}=\sqrt{r_{0}^{2}+r^{2}-2 r_{0} r \cos \left(\theta-\theta_{0}\right)}
$$

To correct the amplitude of the data taken with the wedge removed, a coefficient was formed to accommodate the $\frac{1}{r}$ behavior of the pressure. Thus, the derived direct signal had the form

$$
\left(\frac{r+r_{0}}{r_{d}}\right) R e^{j\left(\phi+\phi_{d}\right)}
$$

Similar operations were performed on the signal data taken with the wedge removed in order to derive a signal corresponding in phase and amplitude to the reflected signal from the wedge. 

The phase change for the reflected signal is given by

$$
\Delta \phi_{r}=k \Delta r_{r}
$$

where now

$$
\begin{aligned}
\Delta r_{r} & =r+r_{0}-r_{r} \\
r_{r} & =\text { distance traveled by reflected signal } \\
& =\left(x^{2}+y^{2}\right)^{\frac{1}{2}}
\end{aligned}
$$

where

$$
\begin{aligned}
& x=r_{0} \cos \theta_{0}-r \cos \theta \\
& y=r_{0} \sin \theta_{0}+r \sin \theta
\end{aligned}
$$

Applying a similar coefficient to correct the amplitude of the reflected signal, the derived reflected signal becomes

$$
\left(\frac{r+r_{0}}{r_{r}}\right) R e^{j\left(\phi+\Delta \phi_{r}\right)}
$$

Next, this, and the derived direct signal, were converted back to rectangular form and subtracted from the 

real and imaginary parts of the superimposed signal stored on the floppy disc.

Finally, the amplitude and phase of the resultant was obtained and compared to the signal reflected from the mirror surface.

Another method of separating the diffracted signal from the compound signal was attempted in the time domain. This was accomplished by time-averaging the digitized compound signal and storing on floppy disc. Then, with the wedge again removed, signals were sent over distances the direct and reflected pulses traveled and likewise digitized, time-averaged, and stored.

These last two sets of data were shifted in time by the computer software to correspond to their arrival times in the compound signal, and then subtracted sample for sample from the stored compound signal data. The resultant was then Fourier transformed as before and again compared to the reference signal from a mirror surface.

Although the above procedures appeared to be above reproach, the results from both methods were totally unrepeatable and hence unreliable. The conclusion drawn here was that since the magnitudes of the direct and the reflected signals were so much larger, of the order of $25 \mathrm{~dB}$, than the differacted signal alone, the computational error inherent in the computer overshadowed the diffracted signal. 

D. Z-AXIS DEPENDENCY

A different type of experiment was run on the $90^{\circ}$ wedge to test the behavior of the diffracted signal as a function of the $z$-axis along the apex. Here, the source was kept in the $z=0$ plane at $\theta_{0}=15^{\circ}$, and $r_{0}=25 \mathrm{~cm}$. The receiver was then moved along the $z$ axis at constant radius of $25 \mathrm{~cm}$ and angle of $255^{\circ}$. The results are plotted in figure 36.

From the theory and data plotted in this figure, it can be seen that the diffracted pressure level is virtually the same as the least-path signal out to approximately $20 \mathrm{~cm}$ from the least-path point $(z=0)$, then decays rapidly. The apex of the wedge is acting essentially as a line source in this region. In other words, only the points along the apex near the $z=0$ plane make any significant contribution to the diffracted pulse. This is the result of the spherical pulse from the point source intercepting the wedge at the closest point of approach and the sources of scatter moving along the wedge first with infinite velocity then slowing to provide reradiation which is strongest in the earliest time, or closest distance.

E. FORWARD DIFFRACTION BY THE PLATE

As in the wedge problem, the analysis of the signal diffracted in the forward direction by the plate was easily accomplished. Here again, the receiver is in the acoustic 



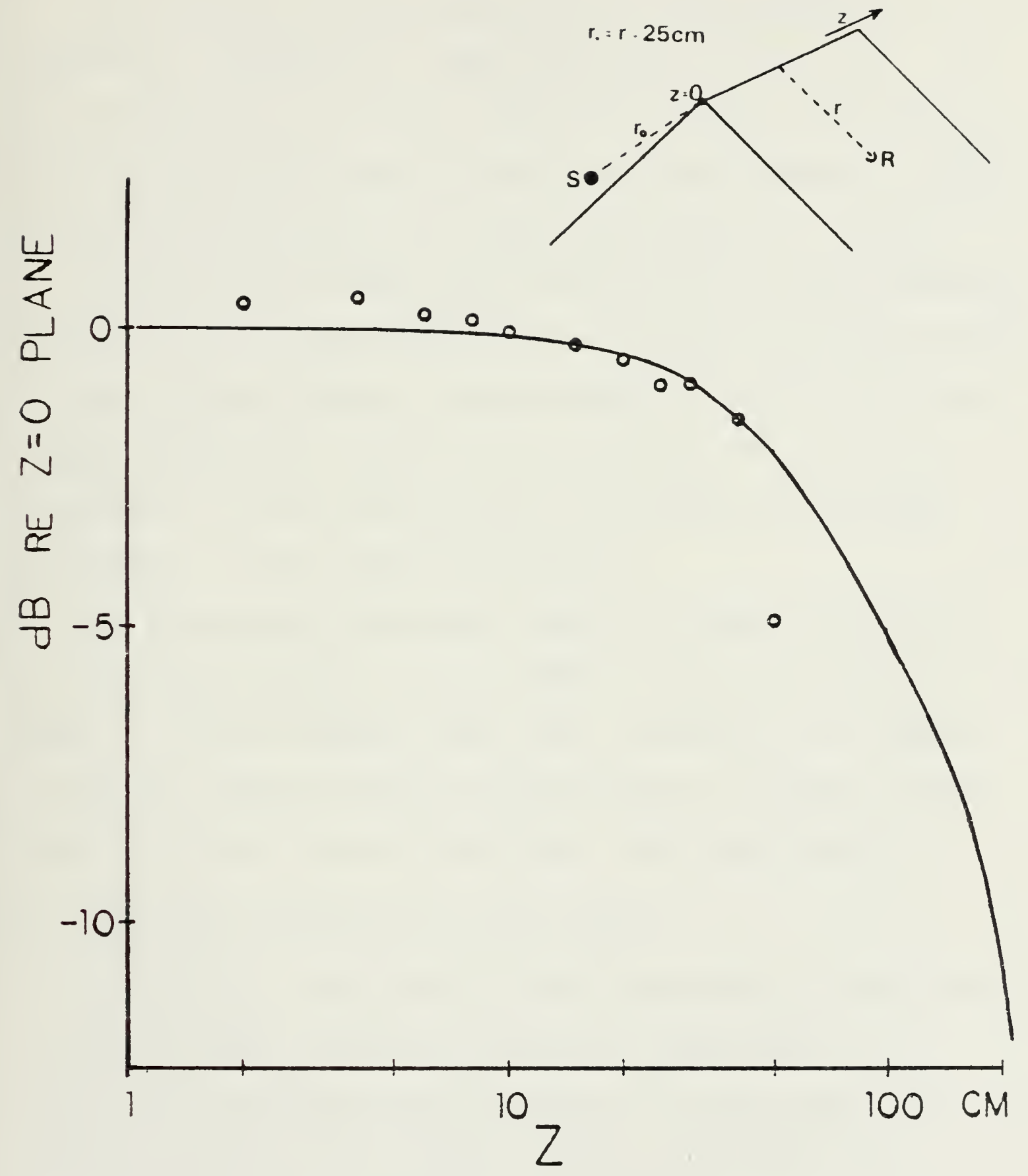

Figure 36. Source at $\theta_{0}=15^{\circ}, r_{0}=25 \mathrm{~cm}, z=0$. Receiver at $^{\circ} \theta=255^{\circ}, r=25^{\circ} \mathrm{cm}, 0 \leqslant z \leqslant 50 \mathrm{~cm}$. 

shadow of the plate and no direct or reflected signals arrive.

The results of these experiments are presented in figures 37 through 46 . Here the source is again at $\theta_{0}=15^{\circ}$, and $r_{0}=25 \mathrm{~cm}$, while the receiver measured in $15^{\circ}$ increments from the far face of the plate, $\theta=360^{\circ}$, to the boundary with the illuminated region, $\theta=210^{\circ}$. Although data points are plotted up to $50 \mathrm{kHz}$ frequency, the validity of the comparison with theory for points above $18 \mathrm{kHz}$ is questionable. As mentioned in section IV, these higher frequencies begin to see the plate as two $90^{\circ}$ wedges due to the plates finite thickness and the short wavelength of the higher frequencies.

An interesting comparison between forward diffraction by the plate and that by the wedge is made in figure 47 . Here, it can be readily seen that a barrier in the shape of the $90^{\circ}$ wedge diffracts approximately $8 \mathrm{~dB}$ more energy into the shadow region than does a thin plate barrier.

\section{F. BACKWARD DIFFRACTION BY THE PLATE}

Once again, the results of the experiments taking data in the backward direction were more complicated than in the forward direction but no more so for the plate than for the wedge. The results of these experiments are presented in figures 48 through 53, for the same source position as in the forward studies.

A polar plot of the acoustic energy diffracted by the plate, as a function of angle about the apex, is presented 



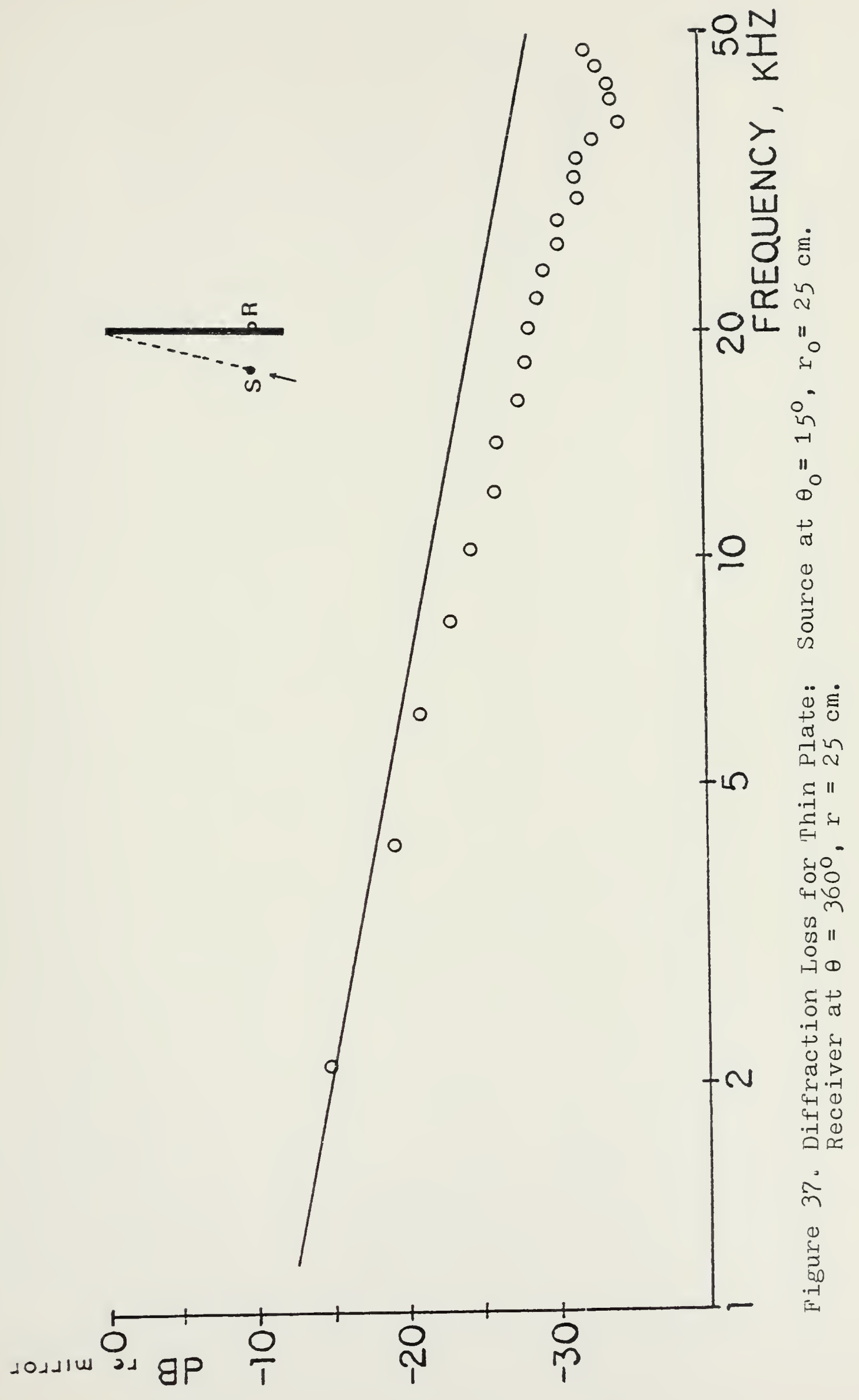





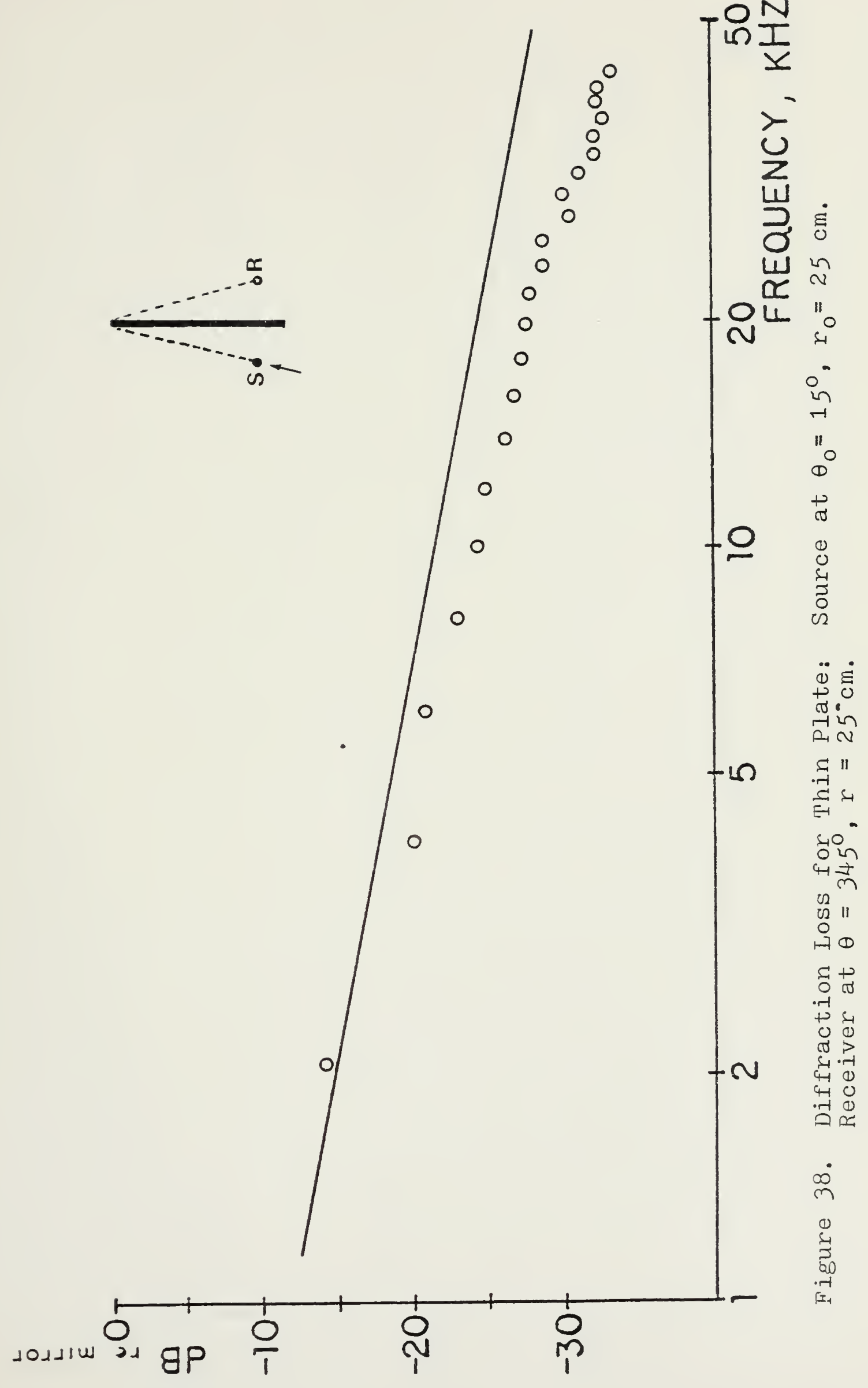





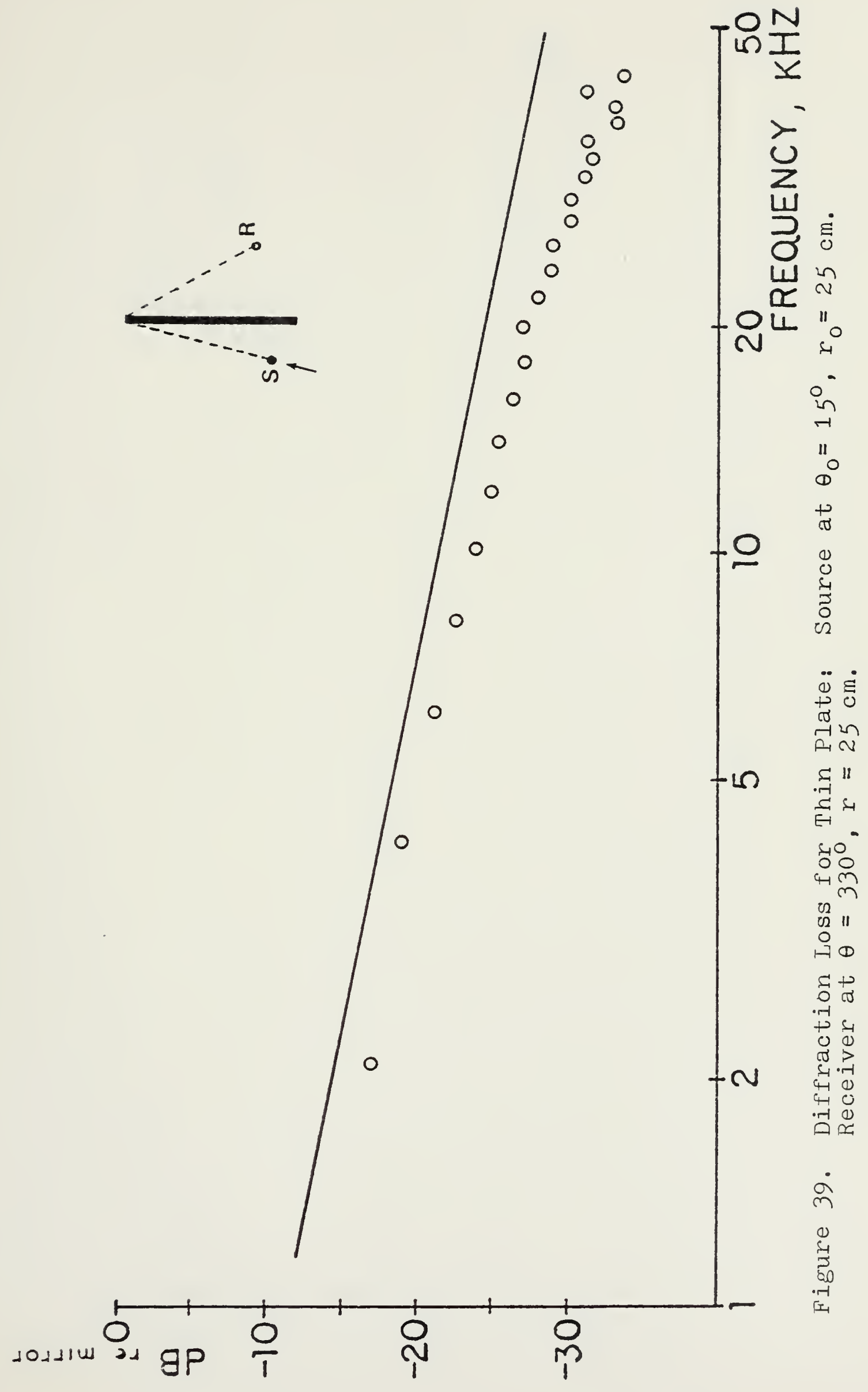





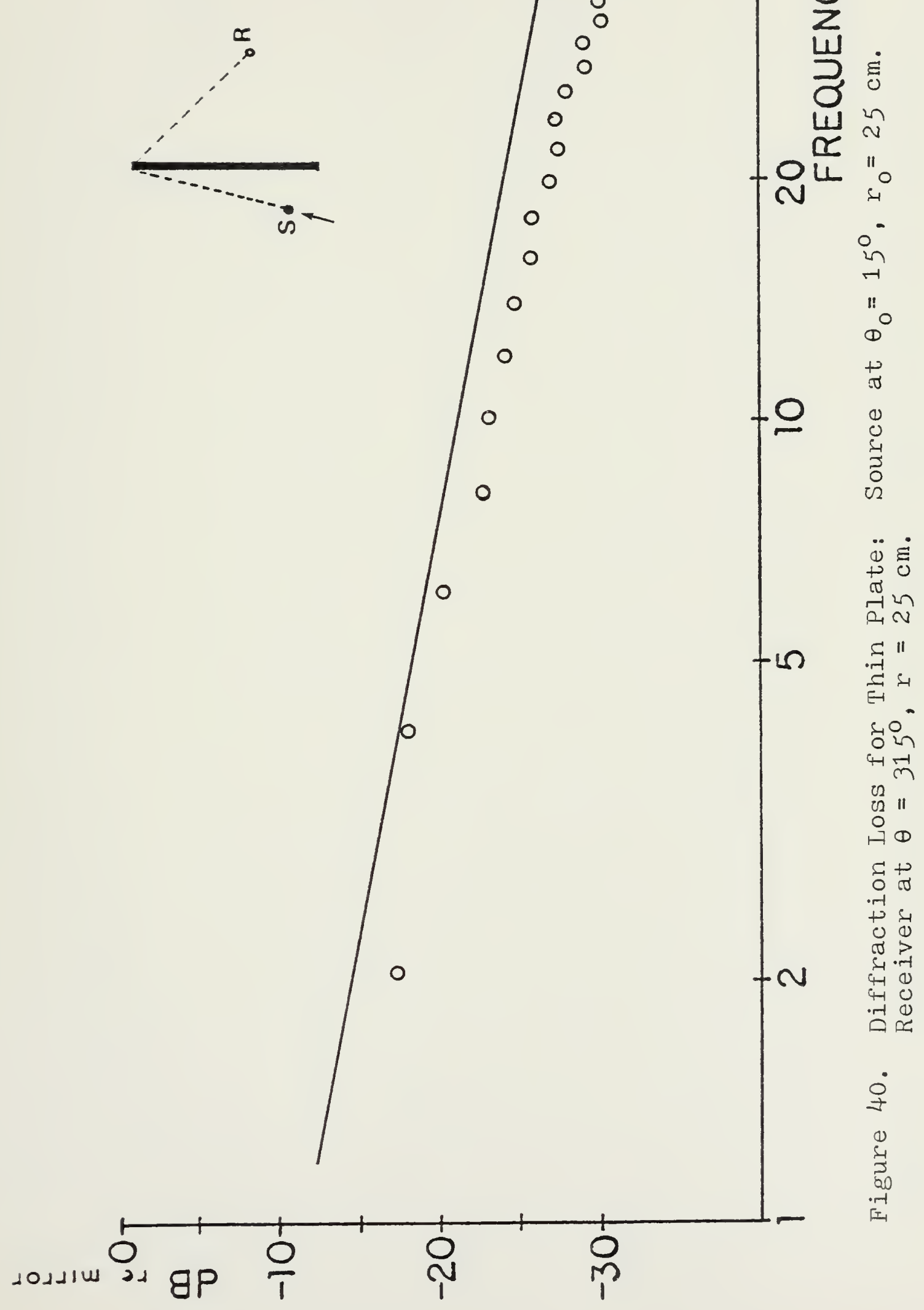





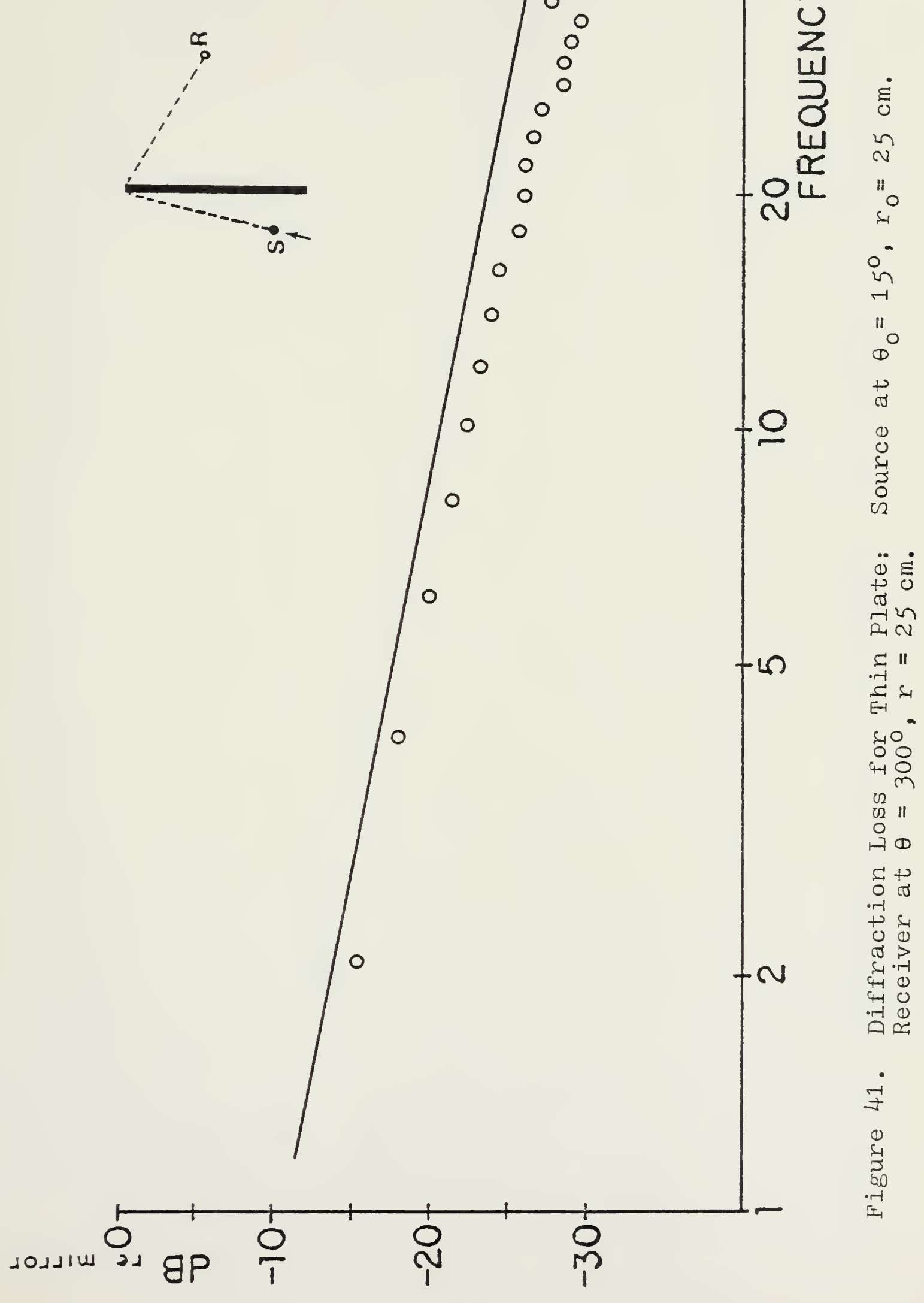





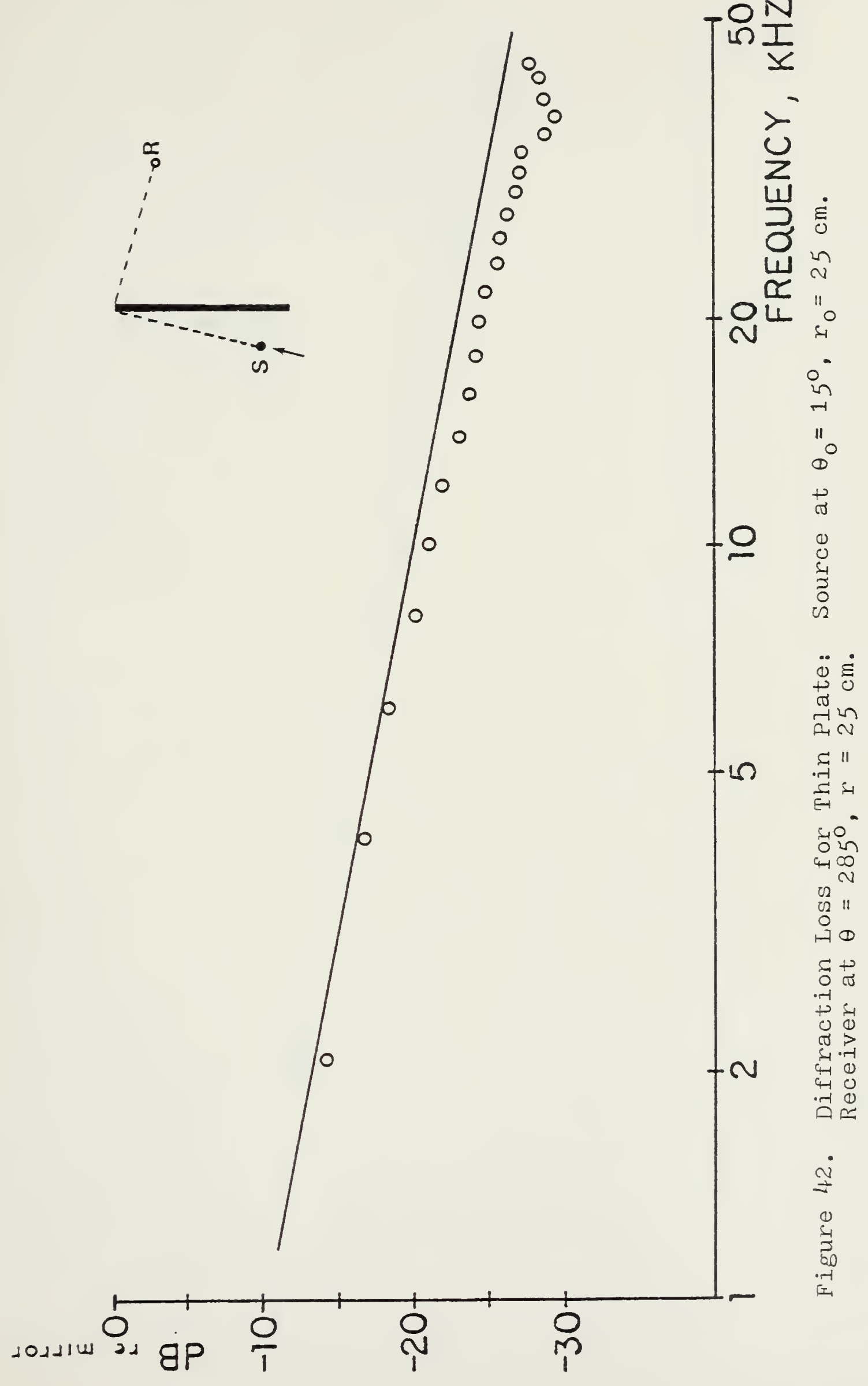





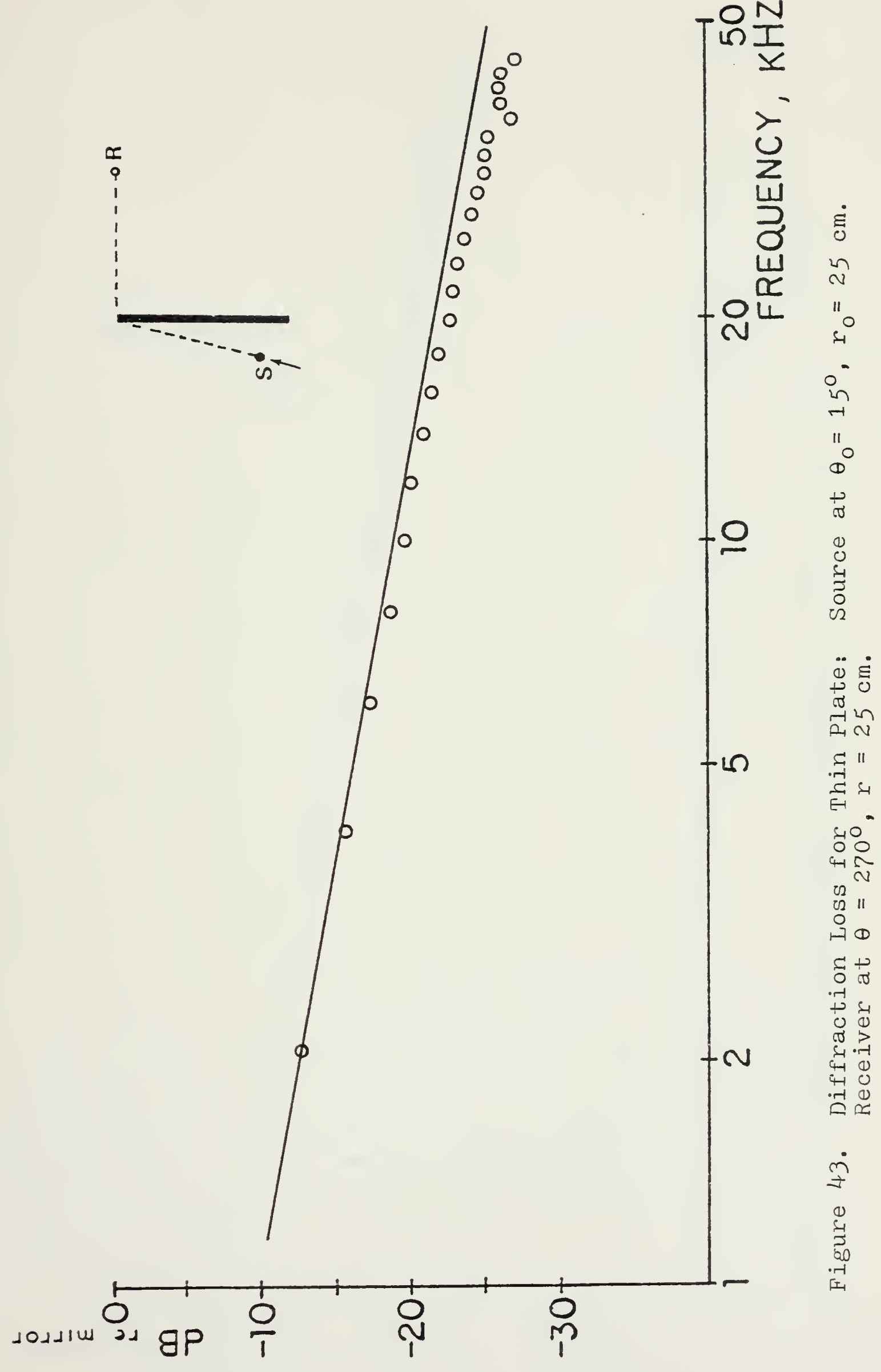





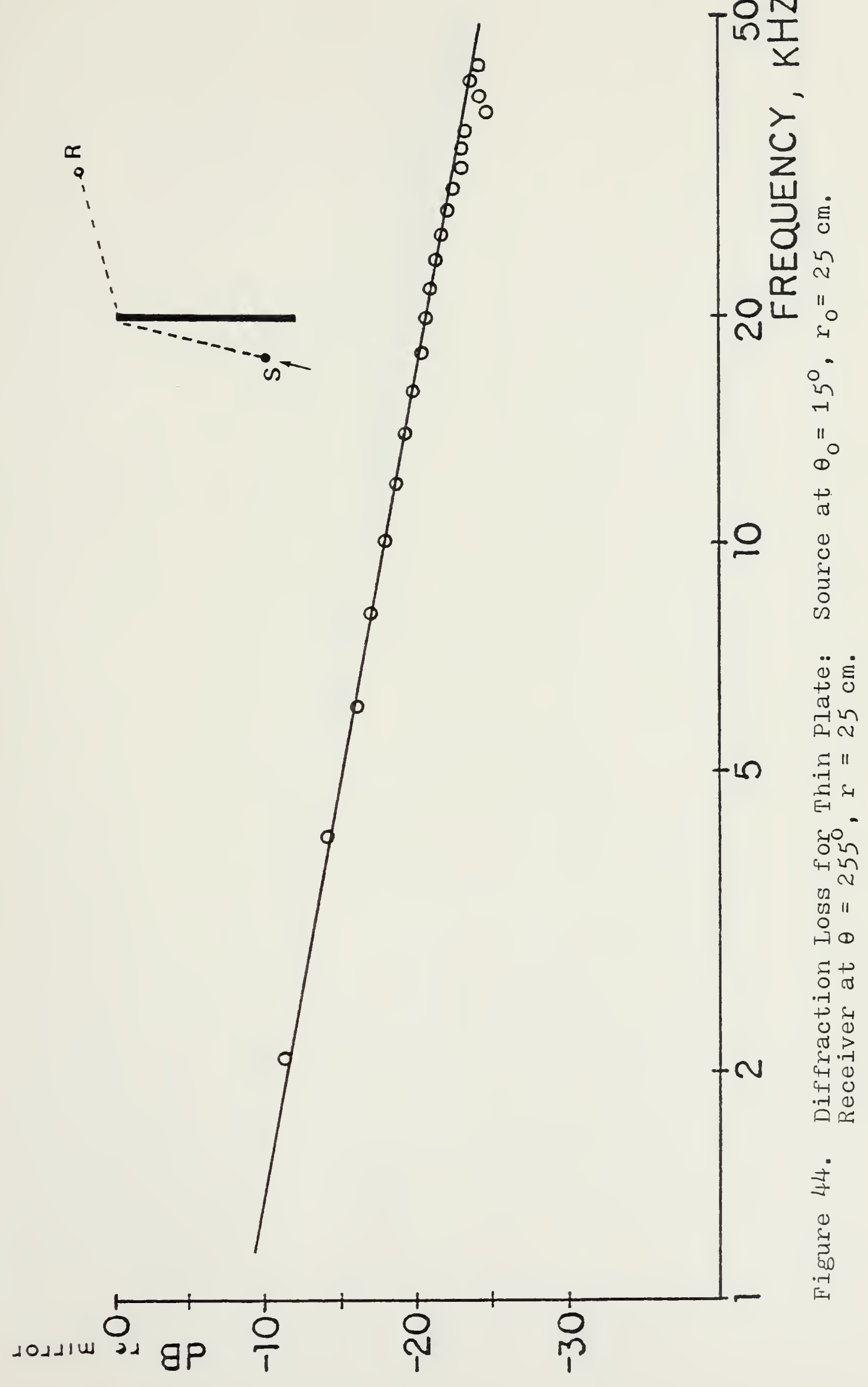





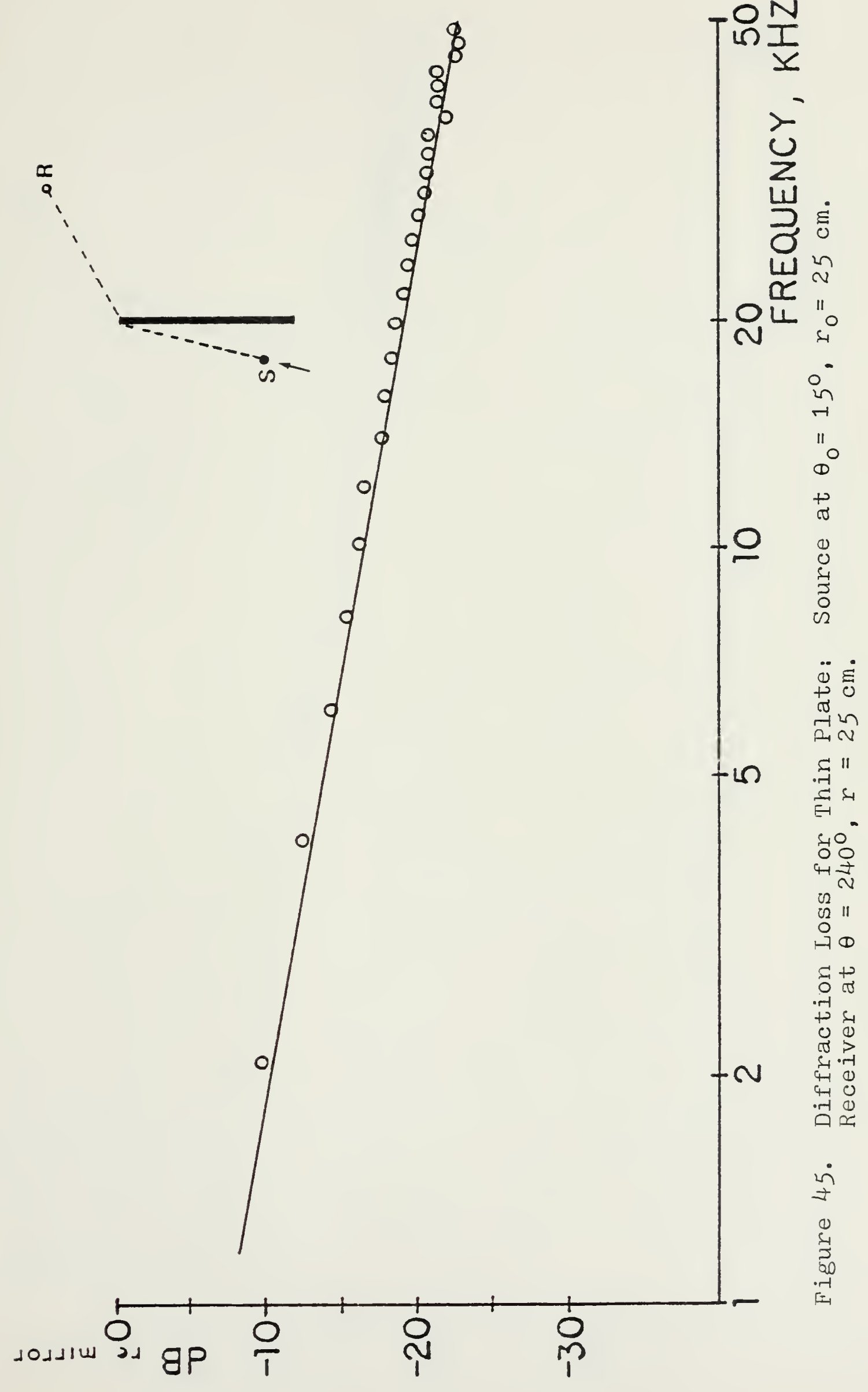





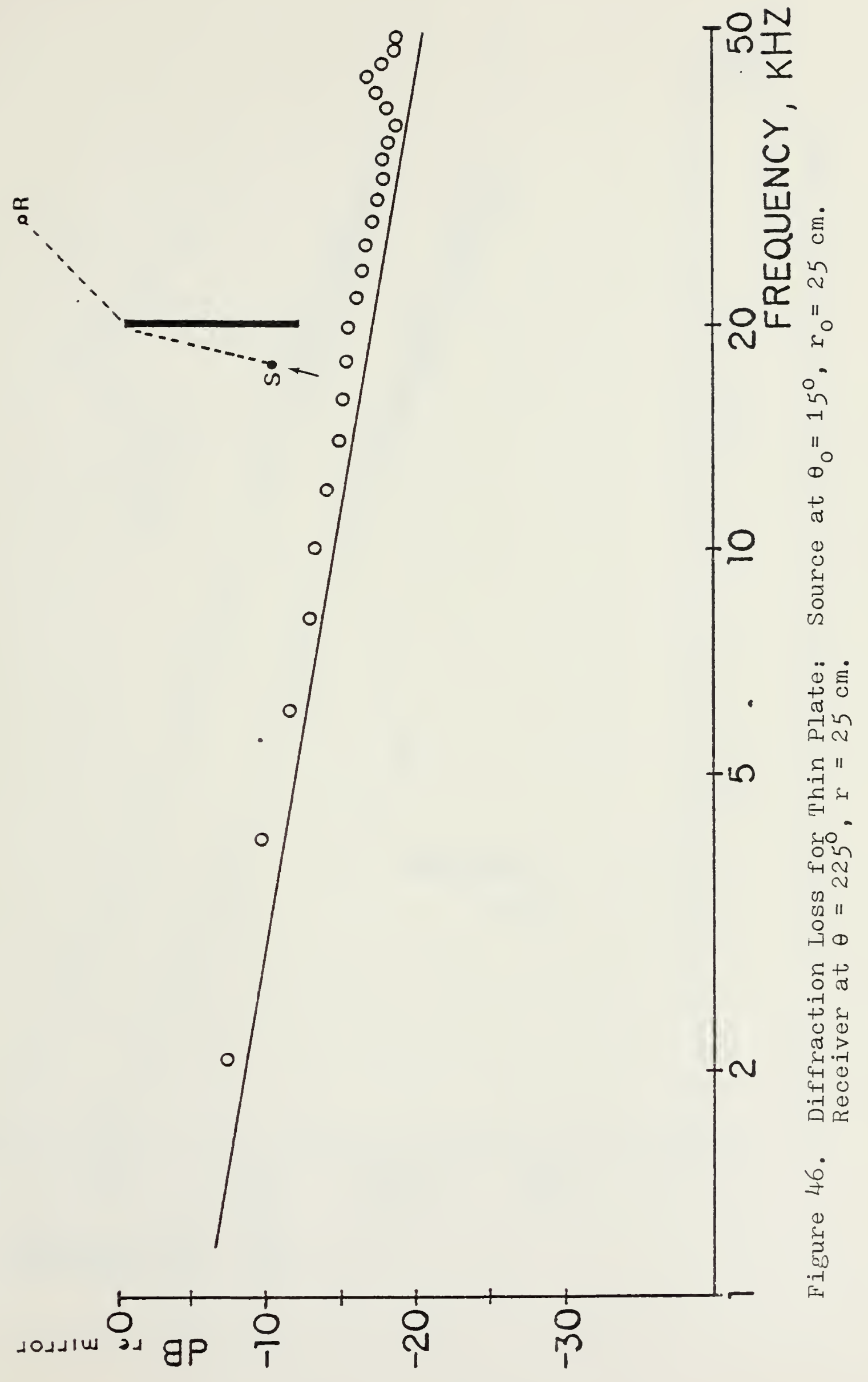





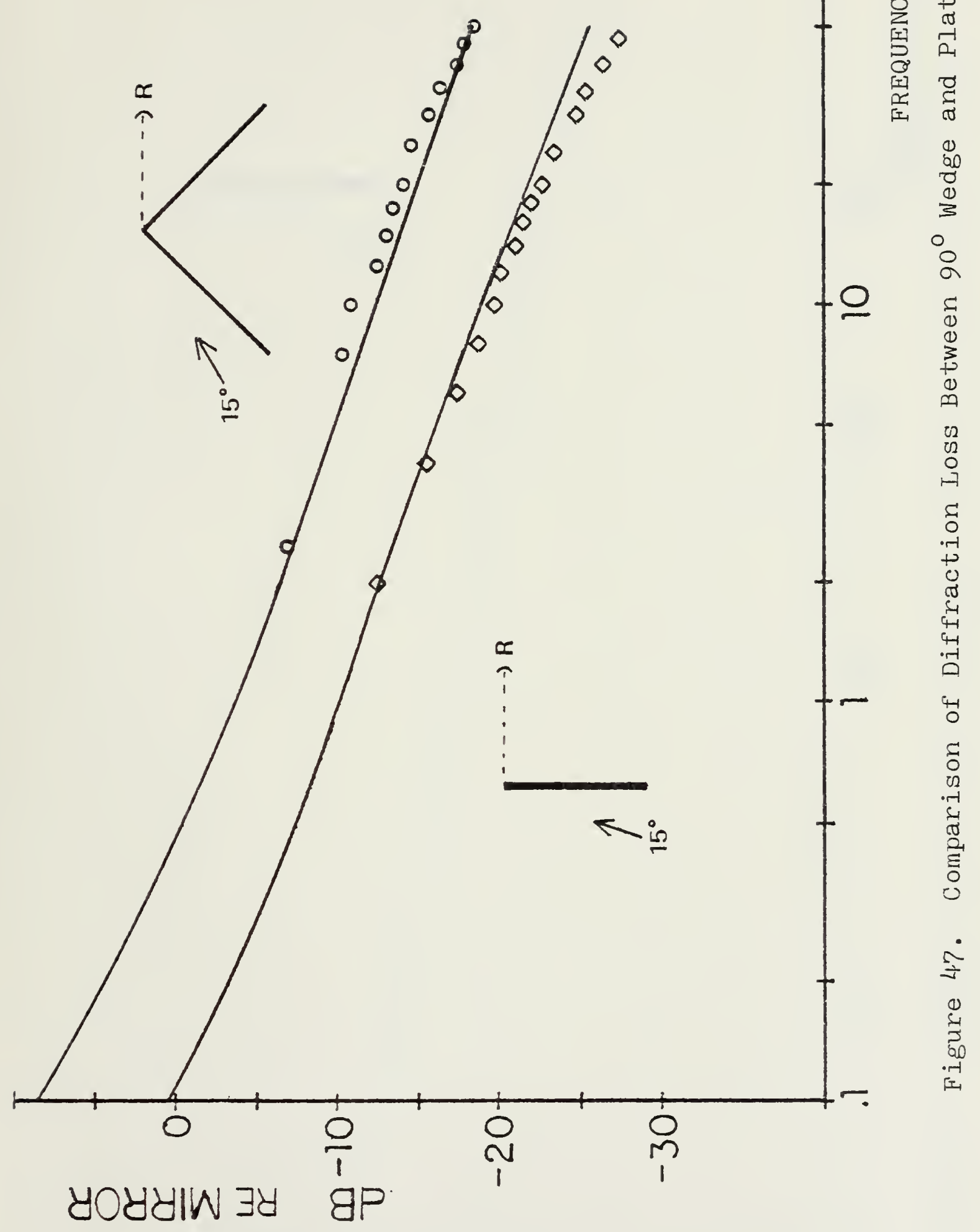





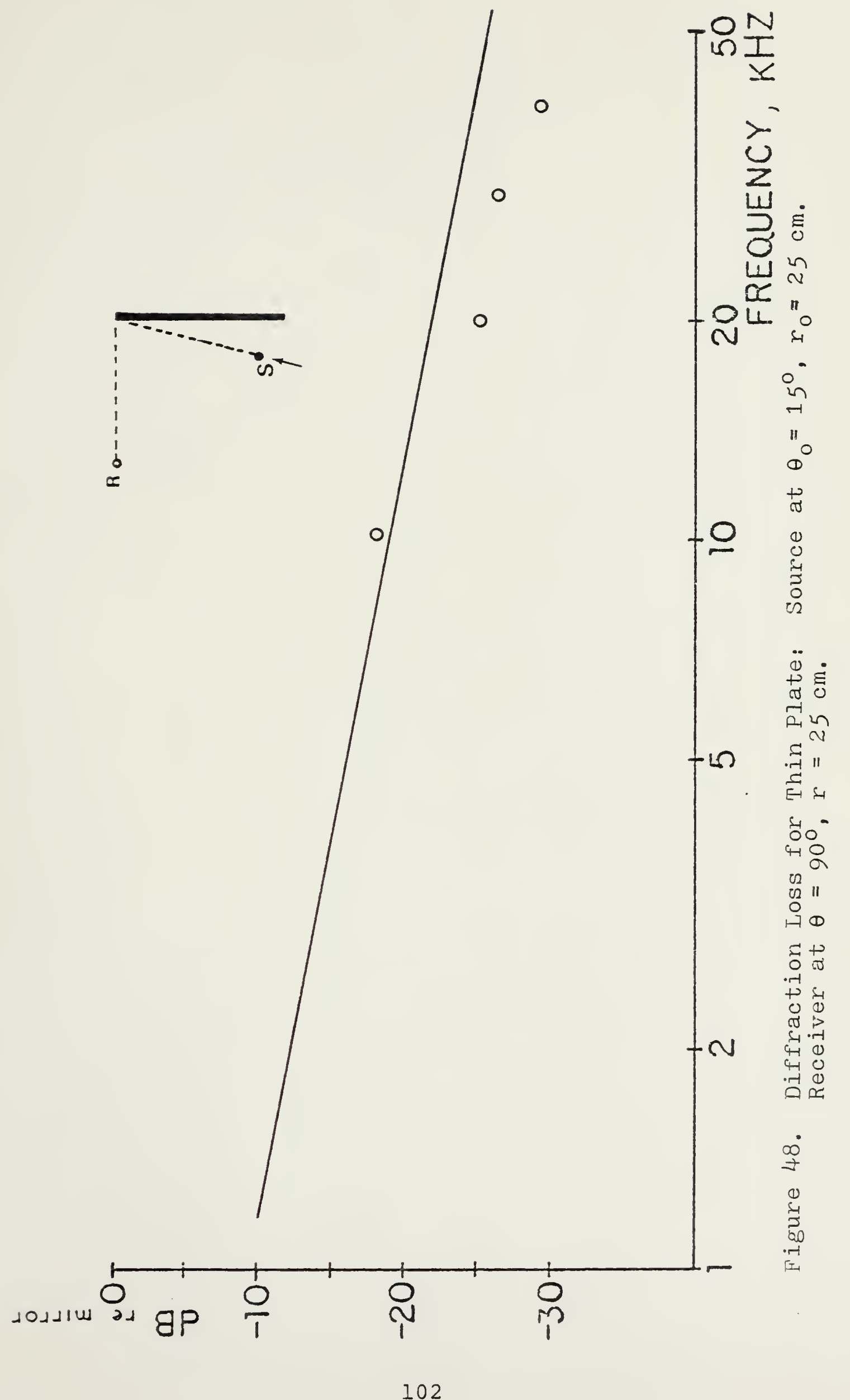





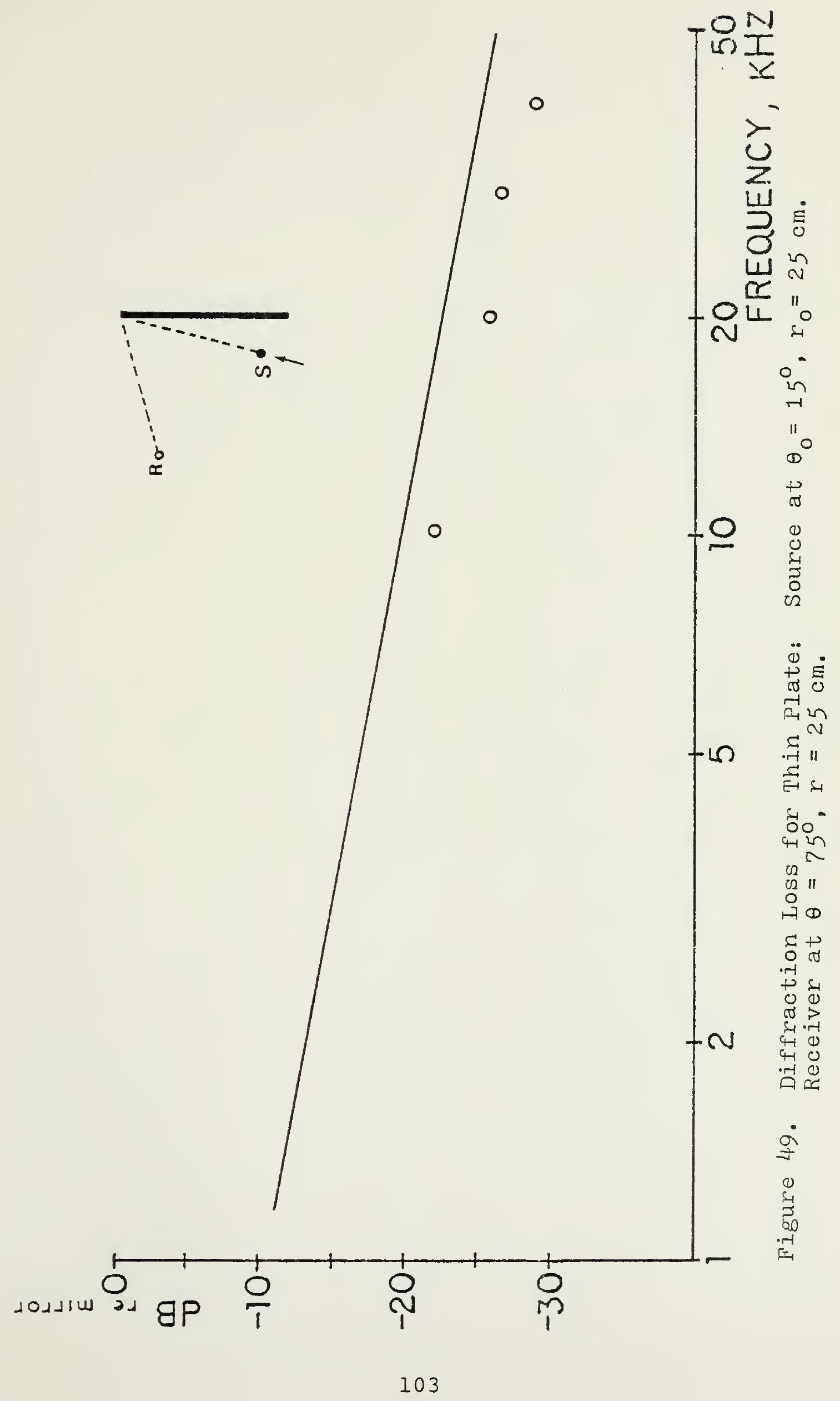





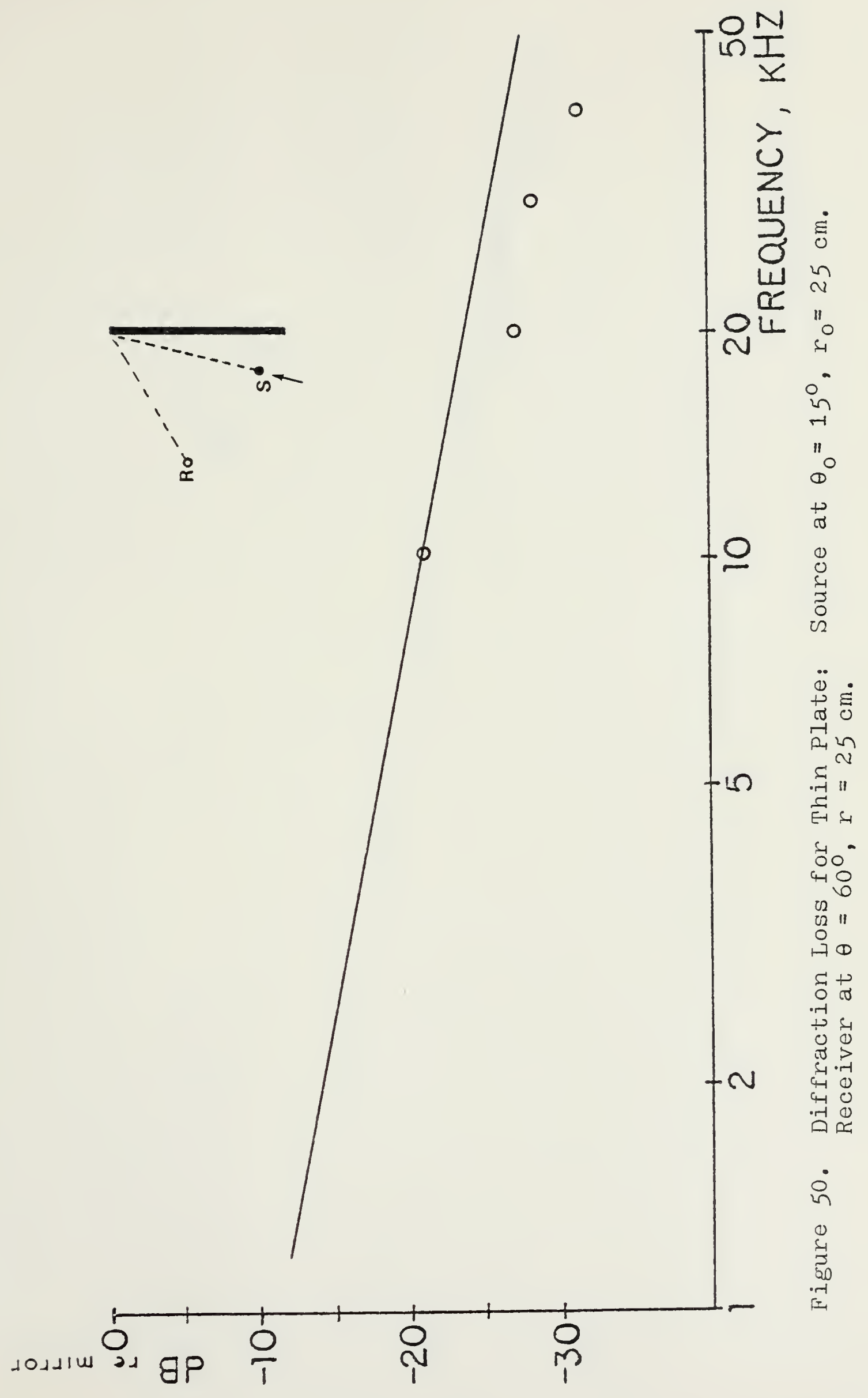





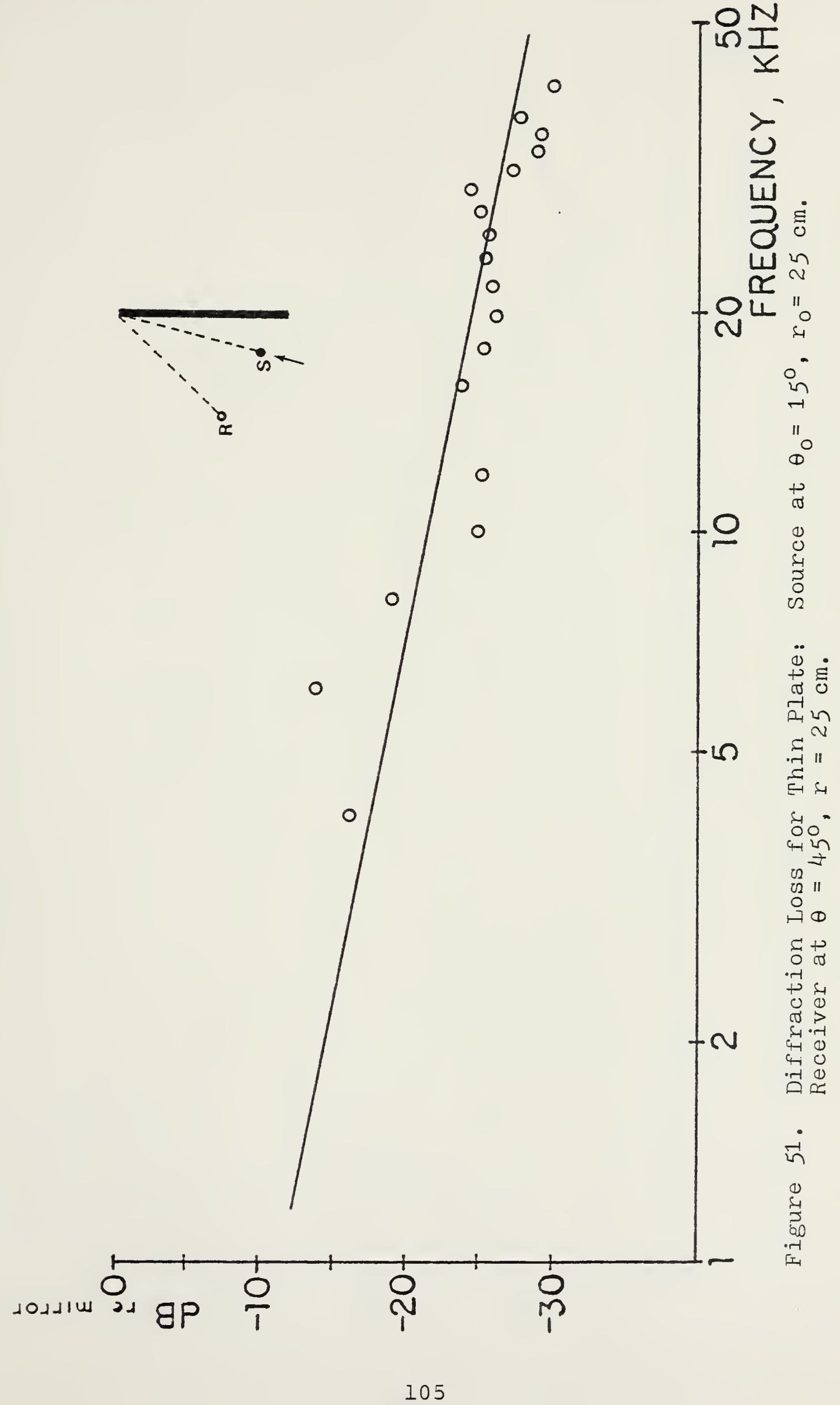





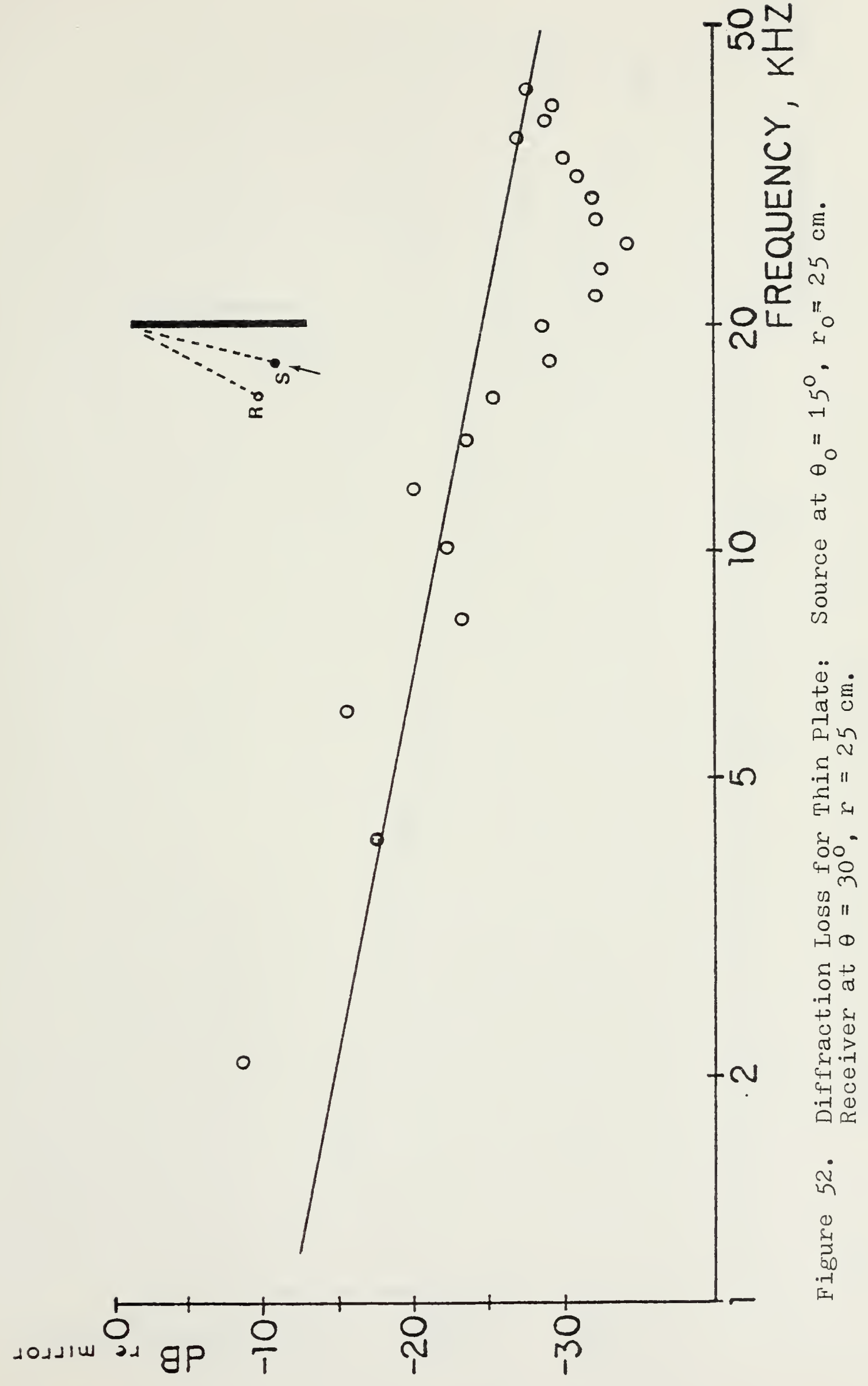





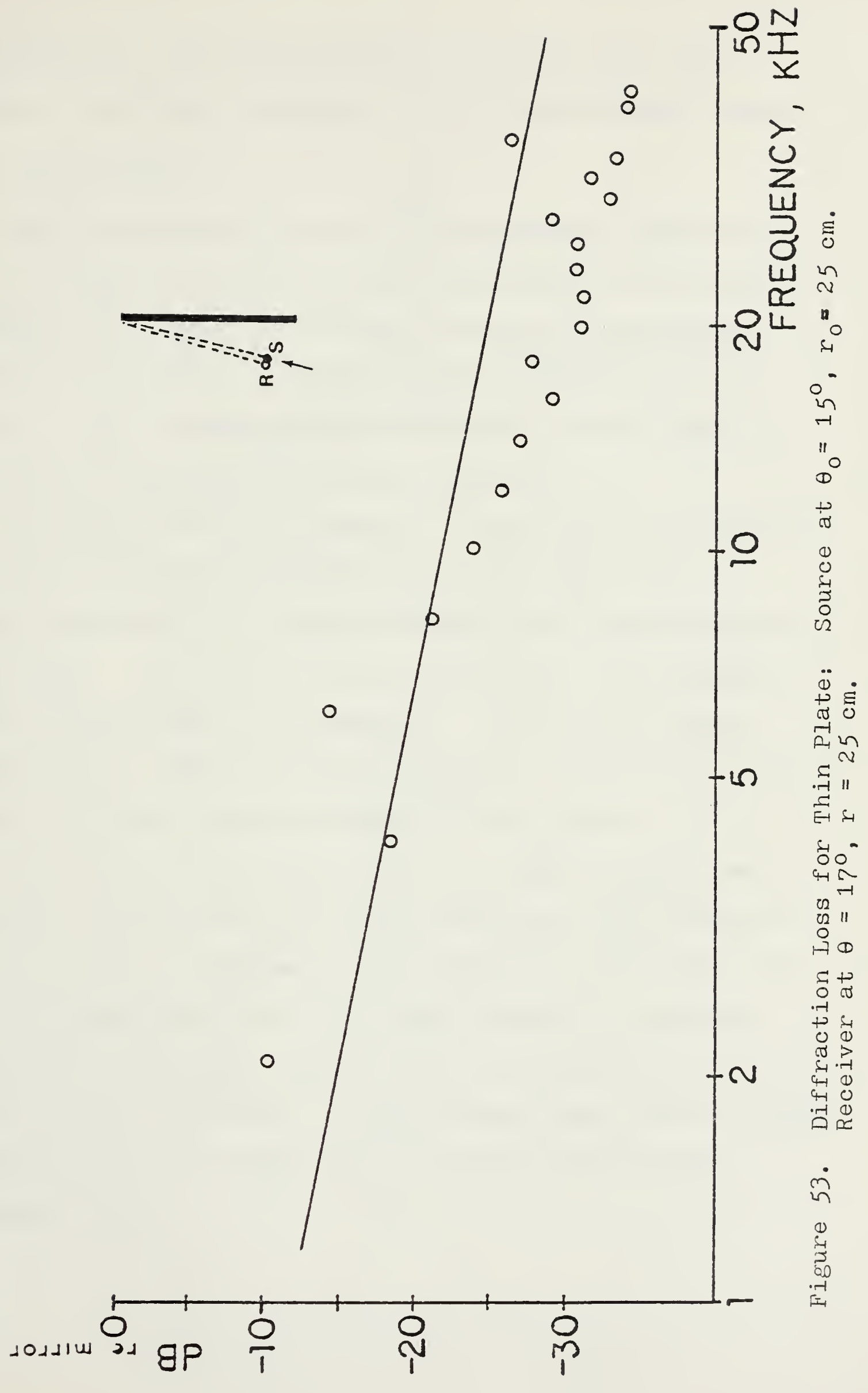



in figure 54. For the frequency of $10 \mathrm{kHz}(\mathrm{kr}=46)$, there is excellent agreement with the Biot-Tolstoy theory.

\section{G. CONCLUSIONS}

The experimental analysis of impulse wave diffraction by a 90 degree wedge and a thin rigid plate in both the forward and backward directions has been accomplished. For the first time, concrete physical evidence of the behavior of diffracted sound waves over a wide range of angles and frequencies has been acquired.

This data has been compared to one of the less popular, yet more satisfying theories available today; that by Biot and Tolstoy, as transformed into the frequency domain by Medwin. As noted in previous sections, the agreement with the B-T theory is remarkable. Only at the higher frequencies, above the $40 \mathrm{kHz}$ response of the probe microphone, does the agreement start to deteriorate.

The data from the plate does not agree quite as well as does the data from the wedge experiments when compared to the theory. The theoretical values were calculated for a two-dimensional plate, i.e., one having no thickness dimension. The experimental plate, however, did indeed have a finite thickness. This thickness had a more profound effect the farther the probe was positioned into the shadow region. 



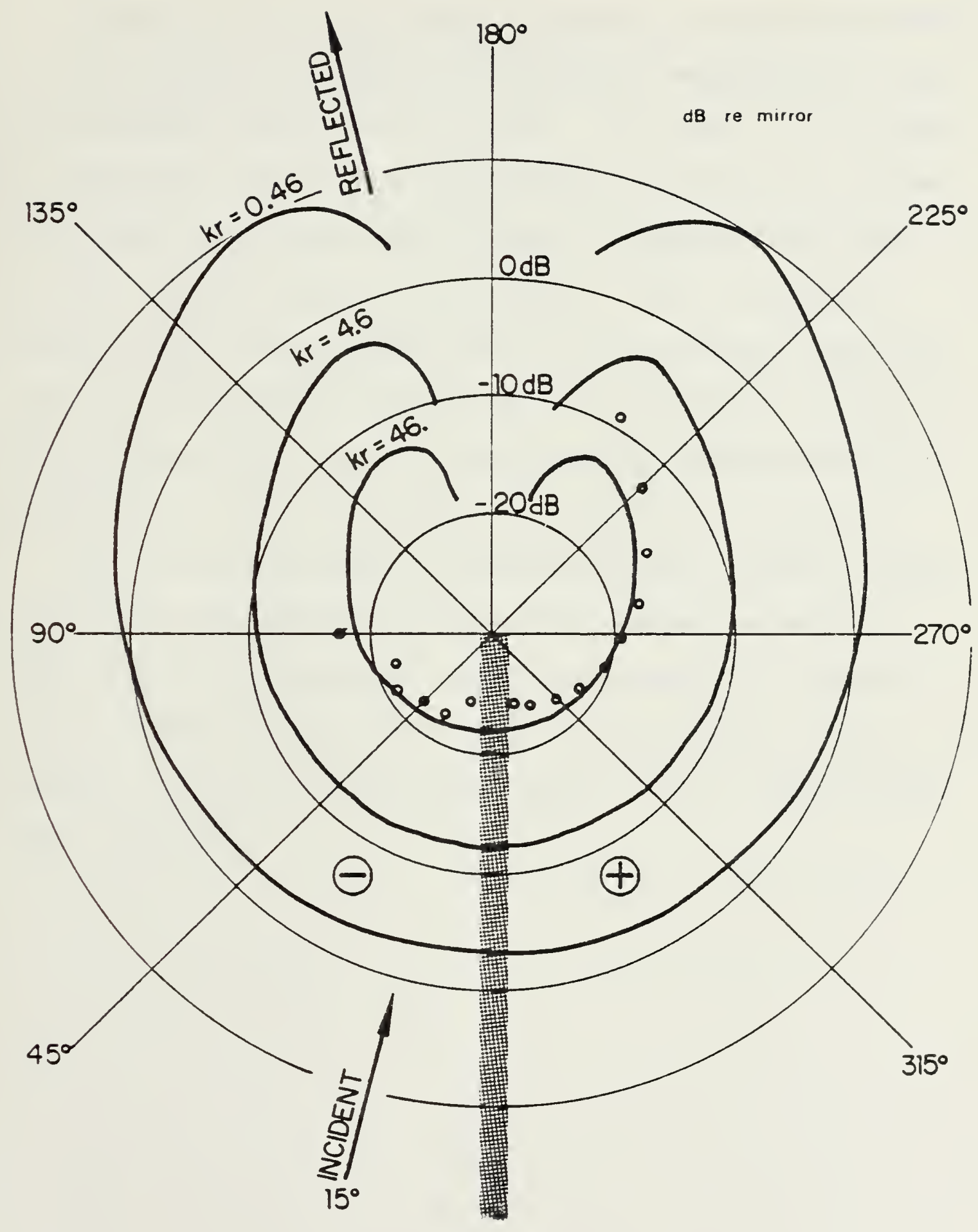

Figure 54. Polar Plot of Diffraction Loss for Thin Plate. Source at $\theta_{0}=15^{\circ}, r_{0}=25 \mathrm{~cm}$. 



\section{RECOMMENDATIONS FOR FUTURE RESEARCH}

Although it is felt that the results of this experiment are in themselves conclusive and highly satisfactory, many more research possibilities arise. In figure 47 it might be noted that the Biot-Tolstoy theory, plotted for values of $\mathrm{kr}$ less than one, tends to show a departure from the 3 AB per octave slope in this region. This suggests a need for more concentrated study at frequencies less than I kHz. This would of course require remodeling the experiment extensively, both for the barrier dimensions and for the dimensions of the source, but would be of interest.

Other possibilities for research would include a study of forward diffraction from multiple wedges; a study of diffraction by a model of a real world barrier, for example a highway noise barrier, or the mid-Atlantic ridge, and perhaps a study of diffraction from a "soft", rather than rigid, barrier. 



\section{BIBLIOGRAPHY}

1. Biot, M. A. and Tolstoy, I., "Formulation of Wave Propagation in Infinite Media by Normal Coordinates with an Application to Diffraction," The Journal of the Acoustical Society of America, v. 29, p. 381-391, 1957.

2. Oberhettinger, F., "On Asymptotic Series for Functions Occurring in the Theory of Diffraction of Waves by Wedges," Journal of Mathematical Physics, v. 34, p. 245255,1956 .

3. Tuzhilin, A. A., "New Representations of Diffraction Fields in Wedge-shaped Regions with Ideal Boundaries," Soviet Physics Acoustics, v. 55, p. 941-955, 1974.

4. Pierce, A. D., "Diffraction of Sound Around Corners and Over Wide Barriers," The Journal of the Acoustical Society of America, v. 55, p. 941-955, 1974 .

5. Jonasson, H. G., "Diffraction by Wedges of Finite Acoustic Impedance with Applications to Depressed Roads," Journal of Sound and Vibration, v. 25,

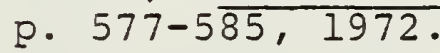

6. Tolstoy, I., Wave Propagation, McGraw-Hill, 1973.

7. Personal communication to author from Dr. H. Medwin.

8. Frederiksen, E., "Condenser Microphones used as Sound Sources," Bruel \& Kjaer Technical Review, No. 3, 1977.

9. Clay, C. S. and Medwin, H., Acoustical Oceanography, Wiley, 1977.

10. Kinsler, L. E. and Frey, A. R., Fundamentals of Acoustics, 2d ed., Wiley, 1962. 

No. Copies

1. Library, Code 0142

Naval Postgraduate School

Monterey, California 93940

2. Department Chairman, Code 61

Department of Physics and Chemistry

Naval Postgraduate School

Monterey, California 93940

3. Professor H. Medwin, Code 6lMd

Department of Physics and Chemistry

Naval Postgraduate School

Monterey, California 93940

4. Lt. Cmar. Joseph H. Bremhorst

204 North 3rd. Street

Clinton, Iowa 52732

5. Manager Anti-Submarine Warfare Systems Project Office, ASW 13

Attn: Car. J.Hagy

Department of the Navy

Washington, D.C. 20360

6. Professor J. Novarini

Av. Cordoba 4190

1188 Cap. Fed.

Buenos Aires

ARGENTINA

7. Director of Defense Research and Engineering I Office of the Secretary of Defense

Washington, D.C. 20301

ATTN: Office, Assistant Director (Research)

8. Professor I. Tolstoy

Knockvennie, Castle Douglas

S. W. SCOTLAND

9. Professor K. Gray, Code 62Gy

Department of Electrical Engineering

Naval Postgraduate School

Monterey, California 93940 

10. Defense Documentation Center

Cameron Station

Alexandria, Virginia 22314

11. Director

Naval Research Laboratory

Washington, D.C. 20375

ATTN : Library, code 2620

12. Office of Naval Research

Arlington, Virginia 22217

ATTN: (Code 480)

ATTN: (Code 460)

ATTN: (Code 102-OS)

AATN: (Code 102IP)

13. Commander

Naval Oceanographic office

Washington, D.C. 20390

ATTN: Code 1640

ATTN: Code 70

14. NODC/NOAA

Rockville, MD 20882

15. Dr. W. G. Neubauer, Code 8132

Naval Research Laboratory

4555 Overlook Ave.

Washington, D. C. 20375

16. Dr. A. J. Rudgers

Naval Research Laboratory

P.O. Box 8337

Orlando, Florida 32856

17. Dr. R. Waterhouse

Code 140

DT NSRDC

Bethesda, Maryland 20084

18. Lt. Masami Kawamura JMSDF

3400 Kamitsuruma, Sagamihara-shi

Kanagawa-ken, JAPAN

19. Lt. Cmar. Ioannis Ioannou

Hellenic Navy Command

Stratopedon Papagou

Athens, GREECE

20. Mrs. Jeanie Savage Code 61

Department of Physics and Chemistry

Naval Postgraduate School

Monterey, California 93940 


Thesis Bremhorst

c.1 tion by rigid wedges

arid plates.

Thesis

B8033:

C.1

Bremhorst

180116

Impul se wave diffrac-

tion by rigid wedges

and plates. 
